\title{
NERVE FIBER DIAMETER MEASUREMENTS USING HEMATOXYLIN AND EOSIN STAINING AND BRIGHTFIELD MICROSCOPY TO ASSESS THE NOVEL METHOD OF CHARACTERIZING PERIPHERAL NERVE FIBER DISTRIBUTIONS BY GROUP DELAY
}

\author{
A Thesis \\ presented to \\ the Faculty of California Polytechnic State University, \\ San Luis Obispo
}

\author{
In Partial Fulfillment \\ of the Requirements for the Degree \\ Master of Science in Biomedical Engineering
}

by

Jorge Arturo Vazquez

August 2014 
(C) 2014

Jorge Arturo Vazquez

ALL RIGHTS RESERVED 


\section{COMMITTEE MEMBERSHIP}

TITLE:

AUTHOR:

DATE SUBMITTED:

COMMITTEE CHAIR: Robert Szlavik, PhD

Associate Professor of Biomedical Engineering

COMMITTEE MEMBER: $\quad$ Scott Hazelwood, PhD

Professor of Biomedical Engineering

COMMITTEE MEMBER: Dan Walsh, $\mathrm{PhD}$

Professor of Biomedical Engineering 


\begin{abstract}
Nerve Fiber Diameter Measurements Using Hematoxylin and Eosin Staining and Brightfield

Microscopy to Assess the Novel Method of Characterizing Peripheral Nerve

Fiber Distributions by Group Delay

Jorge Arturo Vazquez
\end{abstract}

Peripheral neuropathies are a set of common diseases that affect the peripheral nervous system, causing damage to vital connections between various parts of the body and the brain and spinal cord. Different clinical conditions are known to selectively impact various size nerve fibers, which often makes it difficult to diagnose which peripheral neuropathy a patient might have. The nerve conduction velocity diagnostic test provides clinically useful information in the diagnosis of some peripheral neuropathies. This method is advantageous because it tends to be minimally invasive yet it provides valuable diagnostic information. However, this test does not determine characteristics of peripheral nerve fiber size distributions, and therefore does not show any detailed information regarding the nerve fibers within the nerve trunk. Being able to determine which nerve fibers are contributing to the evoked potential within a nerve trunk could provide additional information to clinicians for the diagnosis of specific pathologies of the peripheral nervous system, such as chronic inflammatory demyelinating polyneuropathy or early diabetic peripheral neuropathy. In this study, three rat sciatic nerves are sectioned and stained with hematoxylin and eosin in order to measure the nerve fiber diameters within the nerve trunk. Stained samples are viewed using brightfield microscopy and images are analyzed using ImageJ. Histograms were created to show the frequency of various nerve fiber diameters. The nerve fiber diameters measured during this research are consistent with the range of previously published diameter values and will be used to support continuing research for a novel method to characterize peripheral nerve fiber size distributions using group delay.

Keywords: Group delay, peripheral neuropathy, neuron, hematoxylin, eosin, histology,

nerve fiber size, fiber diameter, distribution conduction velocity, nerve conduction velocity 


\section{ACKNOWLEDGMENTS}

I would like to thank my family, friends and colleagues who have offered their support and encouragement throughout my research and writing. I am extremely thankful for all the guidance and support of Dr. Robert Szlavik, who assisted me and believed in me throughout my entire project. His intelligence and communication helped me to further understand the topic at hand and be able to accomplish the great feat of completing a thesis. Dr. Szlavik has helped this experiment become a great learning experience. Thanks to all the faculty and staff at Cal Poly for all of your support and education.

I would also like to thank Barry-Wehmiller Design Group, Inc. for hiring me and believing in me to finish my research. A special thanks to Yukiko Ikuta for pressuring me to finish my research and guiding me in the right direction. I appreciate your desire to see me succeed in my career. Lastly I would like to thank my parents, brothers and sisters for all of their continuing love and support throughout my entire life. 


\section{TABLE OF CONTENTS}

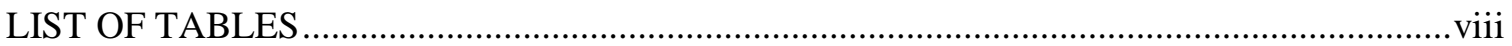

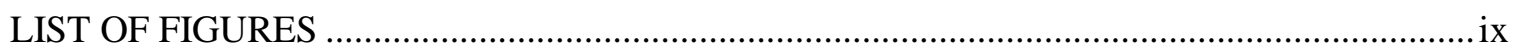

\section{CHAPTER}

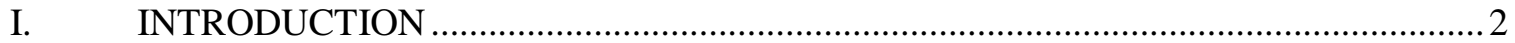

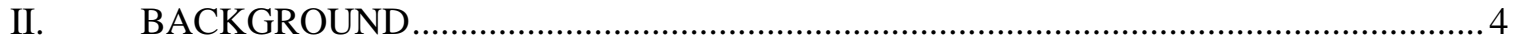

ANATOMY AND PHYSIOLOGY OF A NERVE............................................ 4

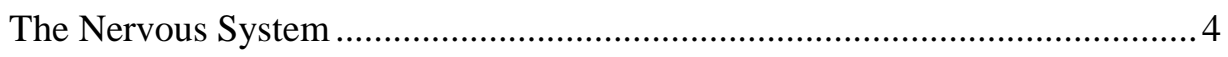

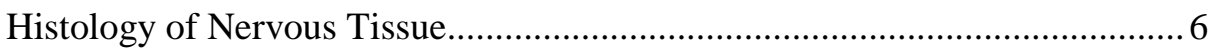

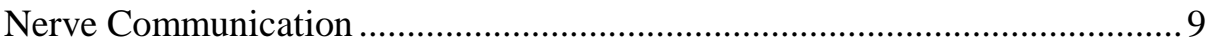

Classification of Nerve Fibers................................................................ 11

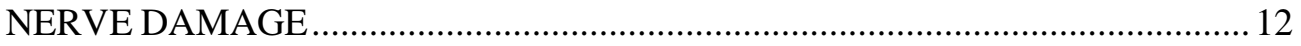

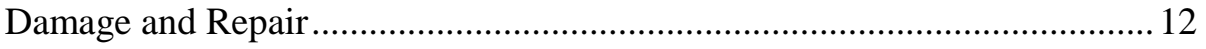

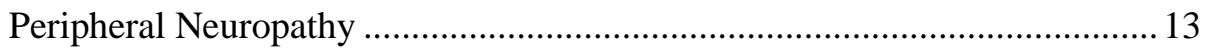

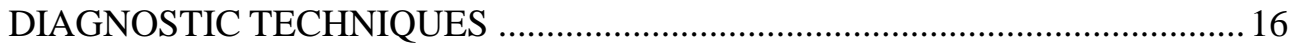

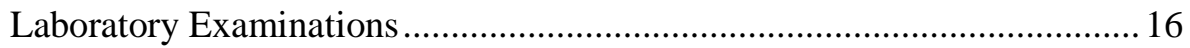

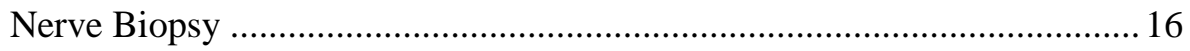

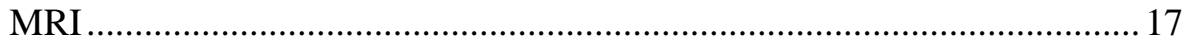

Contact Heat-Evoked Potentials ............................................................. 17

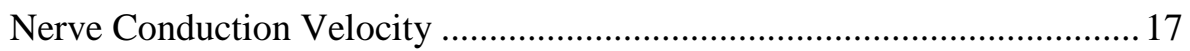


Experimental Model...................................................................... 20

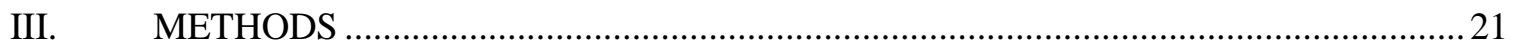

INTRODUCTION TO HISTOLOGY AND H\&E STAINING

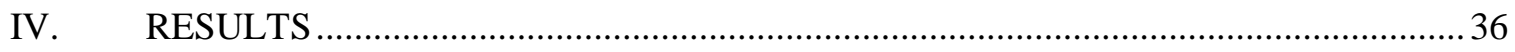

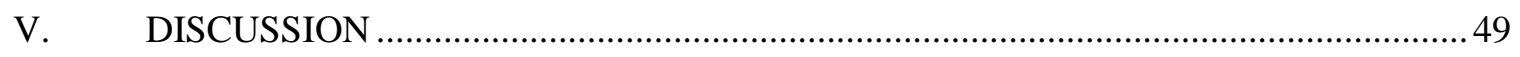

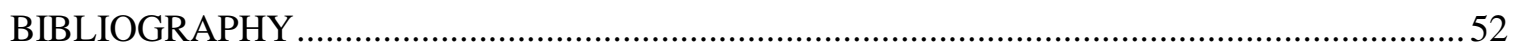

APPENDICES

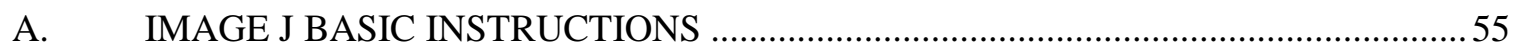

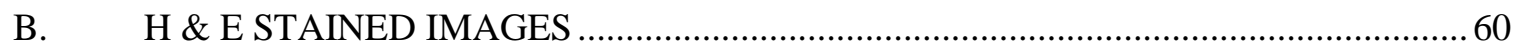

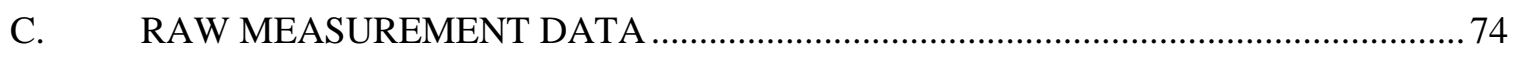

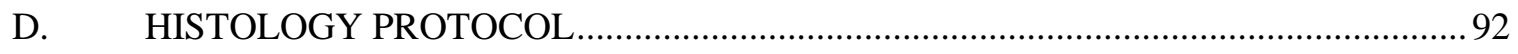




\section{LIST OF TABLES}

Table

1. NERVE FIBER TYPES AND CHARACTERISTICS [7] ................................................. 12

2. (IDIOPATHIC) CAUSES OF SMALL FIBER NEUROPATHY [9] ................................... 15 
Figure

LIST OF FIGURES

1. THE CNS INCLUDES THE BRAIN AND SPINAL CORD, WHILE THE PNS

INCLUDES ALL NERVOUS TISSUE OUTSIDE THE CNS AND CONNECTS

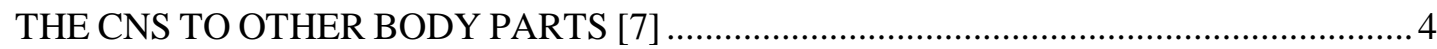

2. STRUCTURE OF A MULTIPOLAR NEURON - A NEURON WITH A LARGE CELL BODY, SEVERAL SHORT DENDRITES, AND A SINGLE LONG AXON. ARROWS

INDICATE THE DIRECTION OF INFORMATION FLOW:DENDRITES --> CELL

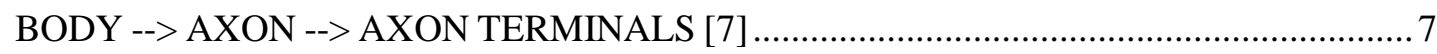

3. SCHWANN CELLS OF THE PNS COMPLETELY SURROUND AXONS AND

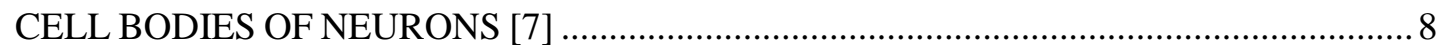

4. TRANSVERSE SECTIONS OF STAGES IN THE FORMATION OF A MYELIN

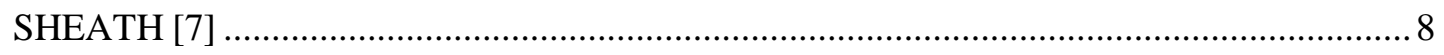

5. DISTRIBUTION OF CHARGES THAT PRODUCE THE RESTING MEMBRANE

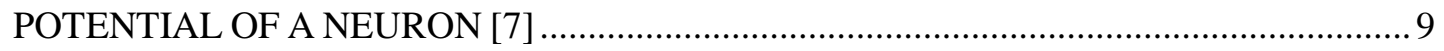

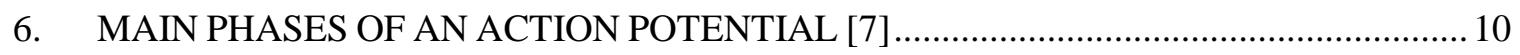

7. ELECTRODE CONFIGURATION FOR NERVE CONDUCTION VELOCITY

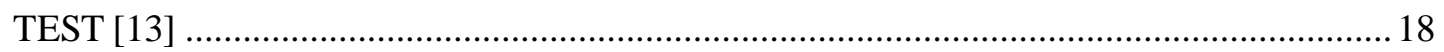

8. PHYSICAL CONFIGURATION FOR THE METHOD PROPOSED BY

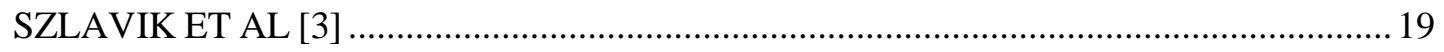

9. VIEW OF THE H \& E STAINING PROCEDURE SET-UP UNDER THE

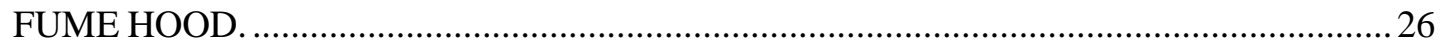

10. BRIGHTFIELD IMAGE OF AN UNUSABLE H \& E STAINED RAT SCIATIC

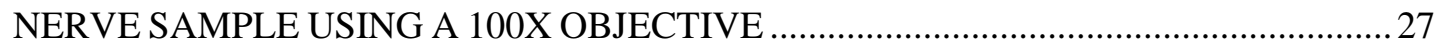


11. H\&E STAINED IMAGE TAKEN USING BRIGHTFIELD MICROSCOPY WITH A

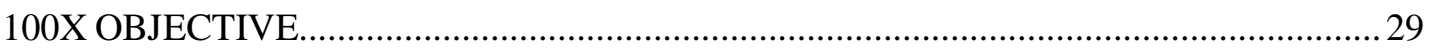

12. EDMUND OPTICS STAGE MICROMETER 1MM/100DIVISIONS ............................... 30

13. EXAMPLE OF SETTING A KNOWN DISTANCE IN IMAGE J (LINE SELECTION

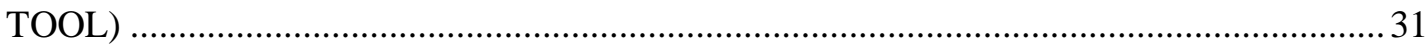

14. EXAMPLE OF SETTING THE SCALE IN IMAGE J (ANALYZE $\rightarrow$ SET SCALE)........... 31

15. EXAMPLE OF OUTLINING THE APPROXIMATE CIRCUMFERENCE OF A NERVE

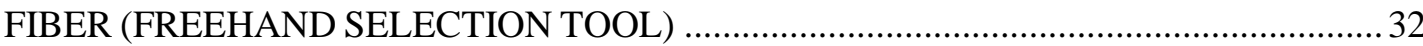

16. ORIGINAL OUTPUT USING THE MICROSOFT OFFICE EXCEL TOOLPAK................ 35

17. ALTERED OUTPUT USING MICROSOFT OFFICE EXCEL TOOLPAK........................... 35

18. HISTOGRAM REPRESENTING DATA FROM SAMPLE 12.028.1.1 .............................. 36

19. HISTOGRAM REPRESENTING DATA FROM SAMPLE 12.028.1.2 ................................ 37

20. HISTOGRAM REPRESENTING DATA FROM SAMPLE 12.028.2.1 .................................. 37

21. HISTOGRAM REPRESENTING DATA FROM SAMPLE 12.028.2.2 …........................... 38

22. HISTOGRAM REPRESENTING DATA FROM SAMPLE 12.028.3.1 ................................. 38

23. HISTOGRAM REPRESENTING DATA FROM SAMPLE 12.028.3.2 _............................. 39

24. HISTOGRAM REPRESENTING DATA FROM SAMPLE 12.028.4.1 ................................. 39

25. HISTOGRAM REPRESENTING DATA FROM SAMPLE 12.028.5.1 ................................. 40

26. HISTOGRAM REPRESENTING DATA FROM SAMPLE 12.028.5.2 ............................... 40

27. HISTOGRAM REPRESENTING DATA FROM SAMPLE 12.029.1.1 ….......................... 41

28. HISTOGRAM REPRESENTING DATA FROM SAMPLE 12.029.1.2 ............................ 41

29. HISTOGRAM REPRESENTING DATA FROM SAMPLE 12.029.2.1 ............................. 42

30. HISTOGRAM REPRESENTING DATA FROM SAMPLE 12.029.4.1 .............................. 42

31. HISTOGRAM REPRESENTING DATA FROM SAMPLE 12.029.5.1 .............................. 43

32. HISTOGRAM REPRESENTING DATA FROM SAMPLE 12.029.5.2 …......................... 43

33. HISTOGRAM REPRESENTING DATA FROM SAMPLE 12.030.1.1 …........................ 44 
34. HISTOGRAM REPRESENTING DATA FROM SAMPLE 12.030.1.2 ............................ 44

35. HISTOGRAM REPRESENTING DATA FROM SAMPLE 12.030.2.1 ............................ 45

36. HISTOGRAM REPRESENTING DATA FROM SAMPLE 12.030.3.1 ............................ 45

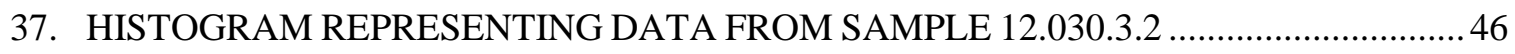

38. HISTOGRAM REPRESENTING DATA FROM SAMPLE 12.030.4.1 ............................ 46

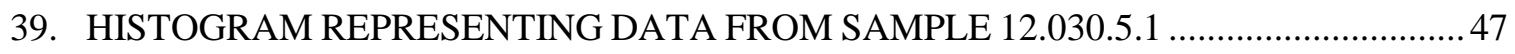

40. HISTOGRAM REPRESENTING COMBINED DATA TAKEN FROM ALL SAMPLES

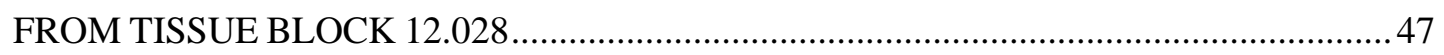

41. HISTOGRAM REPRESENTING COMBINED DATA TAKEN FROM ALL SAMPLES

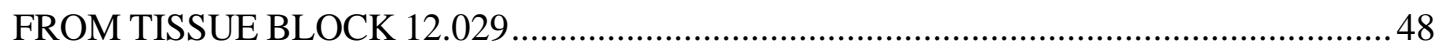

42. HISTOGRAM REPRESENTING COMBINED DATA TAKEN FROM ALL SAMPLES

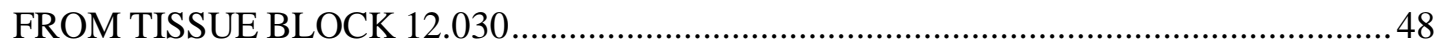

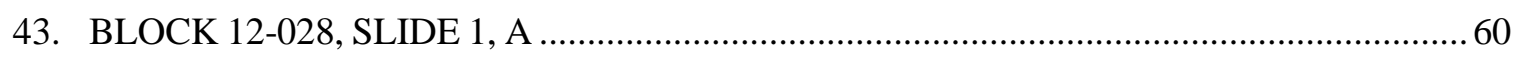

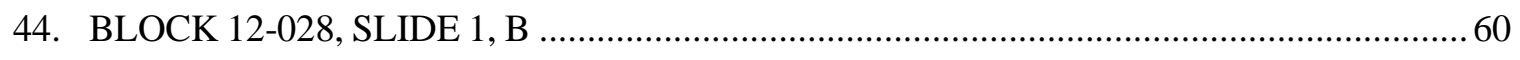

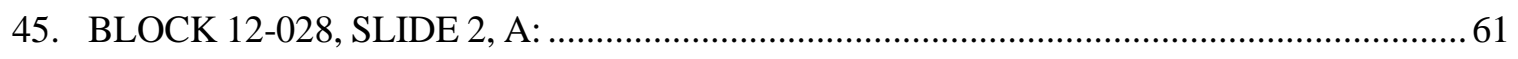

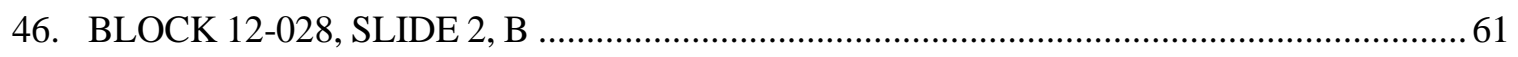

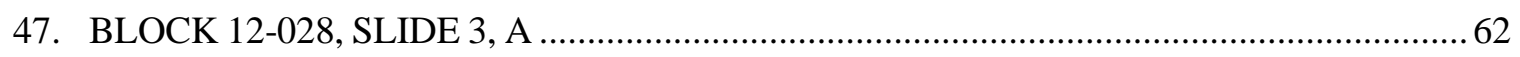

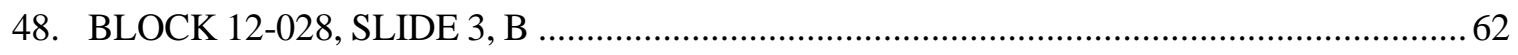

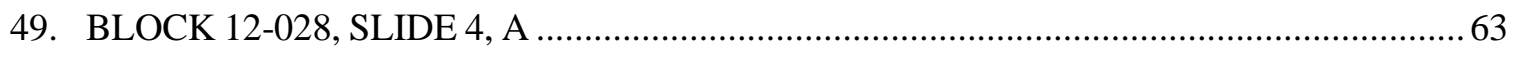

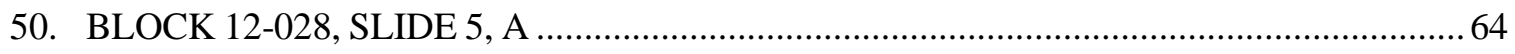

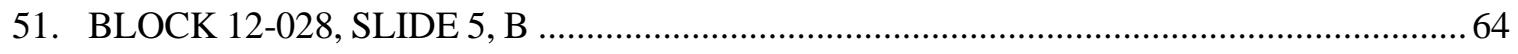

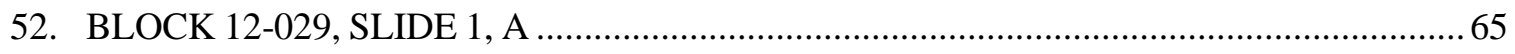

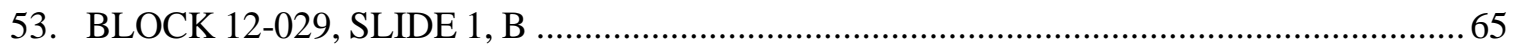

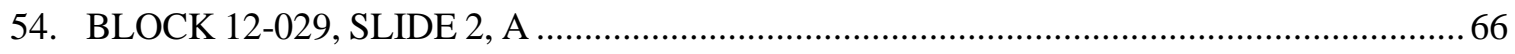

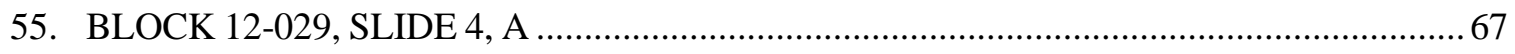

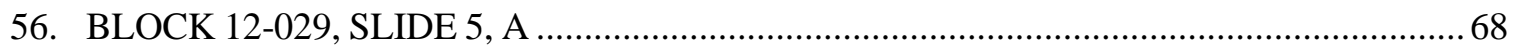




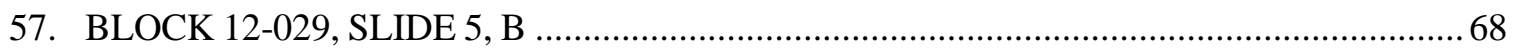

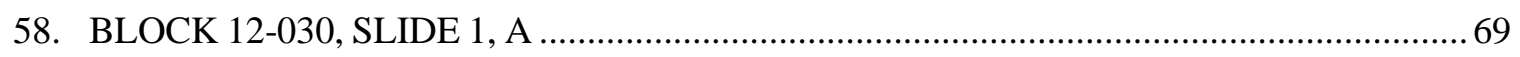

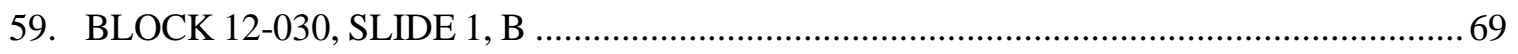

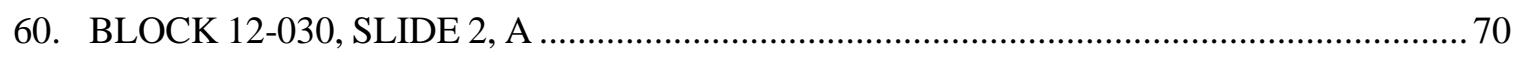

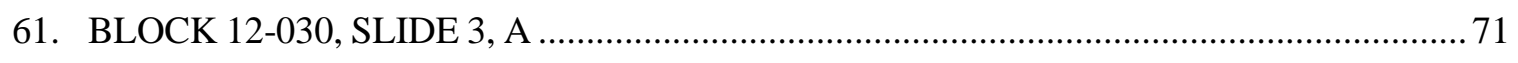

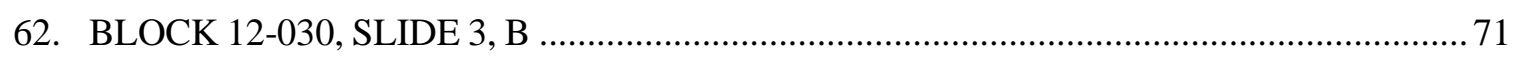

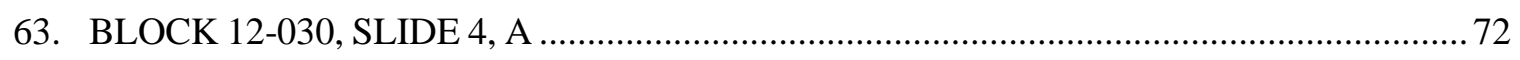

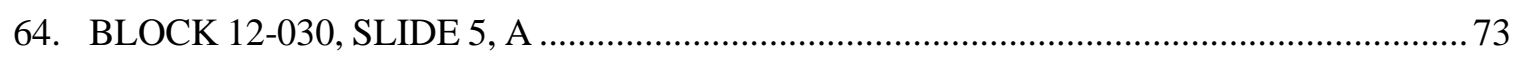




\section{CHAPTER I: INTRODUCTION}

An estimated 15 to 20 million people in the United States over age 40 have some type of peripheral neuropathy [1]. However, many patients are unaware that they have a disease, are not given the correct diagnosis, or the diagnosis is delayed. Currently, treatments do not fix the actual nerve fiber dysfunction or fiber loss, but are focused on treating the underlying medical conditions that cause the neuropathy or are focused on treating symptoms [2]. Some helpful diagnostic techniques include skin biopsies, quantitative sudomotor axon reflex testing (QSART), nerve conduction studies, and electromyography. The skin biopsy and QSART are objective, reproducible, sensitive, and complementary however, these two tests are not widely available. The electrodiagnostic techniques are advantageous because they tend to be minimally invasive yet provide valuable diagnostic information [1].

The nerve conduction velocity test provides clinically useful information in the diagnosis of peripheral neuropathies, such as carpal tunnel syndrome [3]. During the nerve conduction velocity test, surface electrode patches are placed on the surface of the skin to stimulate a specific nerve. One electrode stimulates the nerve while the other records when the compound evoked potential reaches its position. This test determines the speed of conduction of a compound evoked potential down the nerve. While the peripheral nerve conduction velocity test can provide useful information to the clinician regarding the viability of the nerve under study, it is a singleparameter test that yields no detailed information about the characteristics of the functioning nerve fibers within the nerve trunk [3]. Being able to determine information regarding the functioning nerve fibers within the nerve trunk could potentially help differentiate between different clinical conditions such as chronic inflammatory demyelinating polyneuropathy, which selectively impacts larger nerve fibers, or early diabetic peripheral neuropathy, which impacts smaller fibers $[4,5]$. 
Szlavik et al [3] presented a novel technique for estimating the size distribution of contributing nerve fibers which is linearly related to the conduction velocity distribution. The technique is based on an estimation of the group delay between two sets of recording electrodes associated with the individual fibers that contribute to a maximal compound-evoked potential. Incrementally stepping up the stimulation current using small enough steps will, in theory, recruit more and more nerve fibers until a maximal compound evoked potential is reached. With each additional fiber recruited, the output signal increases in magnitude. As the stimulation current continues, the increment is so small that only one new fiber gets recruited with each new step. Then, subtracting the previous compound evoked potential from the current one will yield the potential associated with a single fiber. The group delay information is then used to estimate the diameters of the activated fibers as well as the propagation delays of individual single-fiberevoked potentials to a reference electrode. This process allows for reconstruction of an estimated maximal compound evoked potential, from the individual single-fiber-evoked potentials, at the first recording site. The results of the simulation study demonstrated that the technique could retrieve the fiber size distribution in the presence of recording noise for a wide range of signal-tonoise ratio (SNR) values.

The aforementioned technique has inspired future research regarding the efficacy of the novel method for characterization of peripheral nerve fiber size distributions by group delay. This thesis project will use three extracted rat sciatic nerve, one control model and two chemicallyinduced diabetes models, for the purpose of sectioning and staining the nerves in order to determine the individual nerve fiber diameters. These values will then be compared to results from the study of characterizing peripheral never fiber size distributions by group delay, to determine the efficacy of using this test to characterize different peripheral neuropathies. 


\section{CHAPTER II: BACKGROUND}

\section{ANATOMY AND PHYSIOLOGY OF A NERVE}

\section{THE NERVOUS SYSTEM}

The nervous system is one of the most complex systems of the 11 body systems, made up of a highly organized network of billions of highly specialized nerve cells (neurons) and cells that support the activities of neurons (neuroglia). The structures that make up the nervous system include the brain, spinal cord, cranial nerves and their branches, spinal nerves and their branches, ganglia enteric plexuses, and sensory receptors. These structures can be separated into two main subdivisions: the Central Nervous System (CNS), which consists of the brain and spinal cord, and the Peripheral Nervous System (PNS), which includes all nervous tissue outside the CNS and connects the CNS to other body parts [Figure 1].

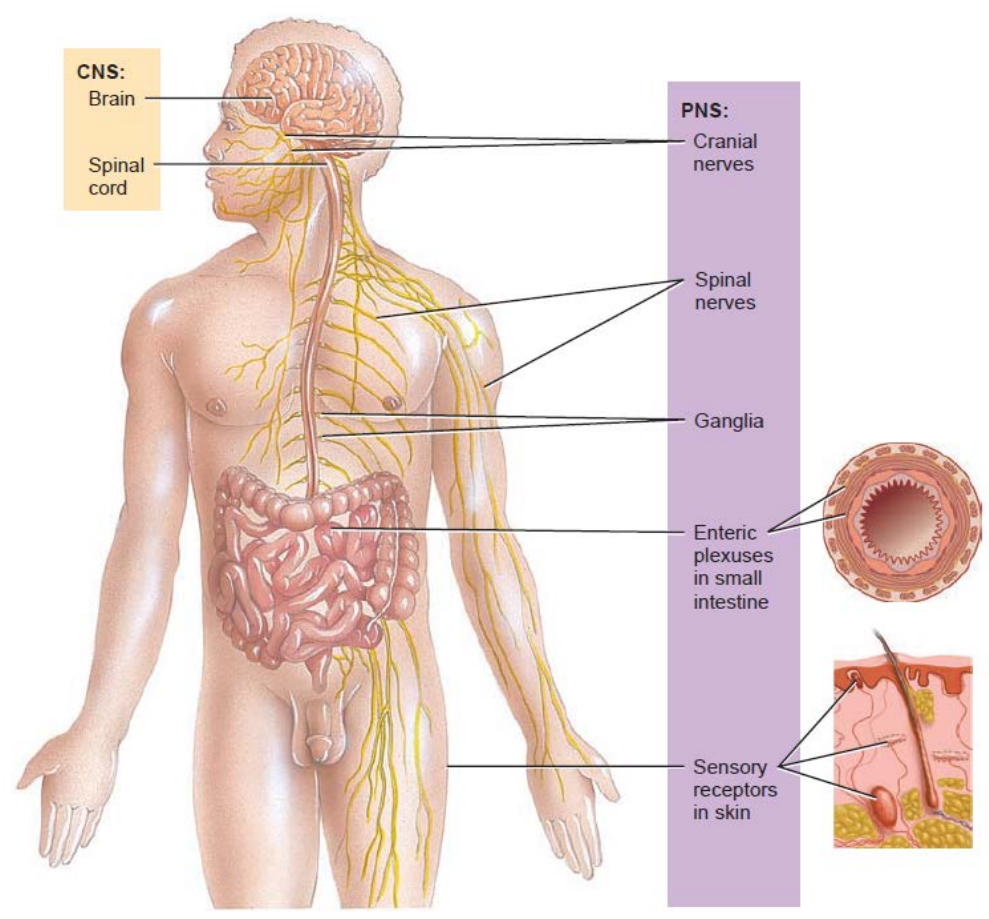

Figure 1. The CNS includes the brain and spinal cord, while the PNS includes all nervous tissue outside the CNS and connects the CNS to other body parts [7] 
The nervous system carries out a complex array of tasks which can be grouped into three basic functions: sensory, integrative, and motor.

- Sensory function: Sensory receptors at the ends of peripheral neurons detect internal stimuli, such as an increase in blood acidity, and external stimuli, such as a raindrop landing on your arm. Sensory receptors convert their information into nerve impulses, which are then carried to the brain and spinal cord through the cranial and spinal nerves of the PNS.

- Integrative function: Once the signals reach the CNS, they are integrated (processed), creating sensations, adding to memory, or helping produce thoughts. An important integrative function is perception, the conscious awareness of sensory stimuli.

- Motor function: Following integration, conscious or subconscious decisions are made and then acted upon by means of motor functions. The nervous system may elicit an appropriate motor response by activating effectors (muscles and glands) through cranial and spinal nerves. Stimulation of the effectors causes muscles to contract and glands to secrete. The motor portion of the PNS can be subdivided into the somatic nervous system (SNS), autonomic nervous system (ANS), and enteric nervous system (ENS) [6, 7].

The SNS consists of sensory neurons that convey information from somatic receptors in the head, body wall, and from receptors for the special senses of vision, hearing, taste, and smell to the CNS; and motor neurons that conduct impulses from the CNS to skeletal muscles only. The action of the SNS is considered voluntary since the motor responses can be consciously controlled. The ANS consists of sensory neurons that convey information from autonomic sensory receptors, primarily in visceral organs, to the CNS; and motor neurons that conduct nerve impulses from the CNS to smooth muscle, cardiac muscle, and glands. The action of the ANS is considered involuntary because motor responses are not normally under conscious control. The 
ENS consists of neurons that extend most of the length of the gastrointestinal (GI) tract. Sensory neurons of the ENS monitor chemical changes within the GI tract as well as the stretching of its walls. The ENS is considered the "brain of the gut", and its action is considered involuntary [7].

\section{HISTOLOGY OF NERVOUS TISSUE}

Nerve tissue consists of two types of cells: neurons and neuroglia. Neurons sense certain types of changes in their surroundings and respond by transmitting nerve impulses along cellular processes to other neurons or to muscles or glands, resulting in coordination, regulation, and integration with many body functions. Neurons provide most of the unique functions of the nervous system, such as sensing, thinking, remembering, controlling muscle activity, and regulating glandular secretions. The abundant neuroglia support and bind the components of nervous tissue, carry on phagocytosis, and help supply nutrients to neurons by connecting them to blood vessels [6].

Neurons vary considerably in size and shape, but they all have three basic parts: a cell body, dendrites, and an axon [Figure 2]. The cell body, or soma, contains a large spherical nucleus surrounded by cytoplasm that includes typical cellular organelles such as mitochondria, lysosomes, a Golgi apparatus, and many microtubules. Extending from the soma are processes called nerve fibers. Most neurons have two kinds of never fibers, dendrites and an axon. Dendrites are usually highly branched processes extending from the cell body and are the receiving or input portions of a neuron. The cell body can have many dendrites, but only one axon extends from the soma. The axon is a long, thick, cylindrical projection that arises from the cell body at a cone-shaped elevation called the axon hillock. The axon is responsible for propagating nerve impulses away from the cell body toward another neuron, a muscle fiber, or a gland cell. 


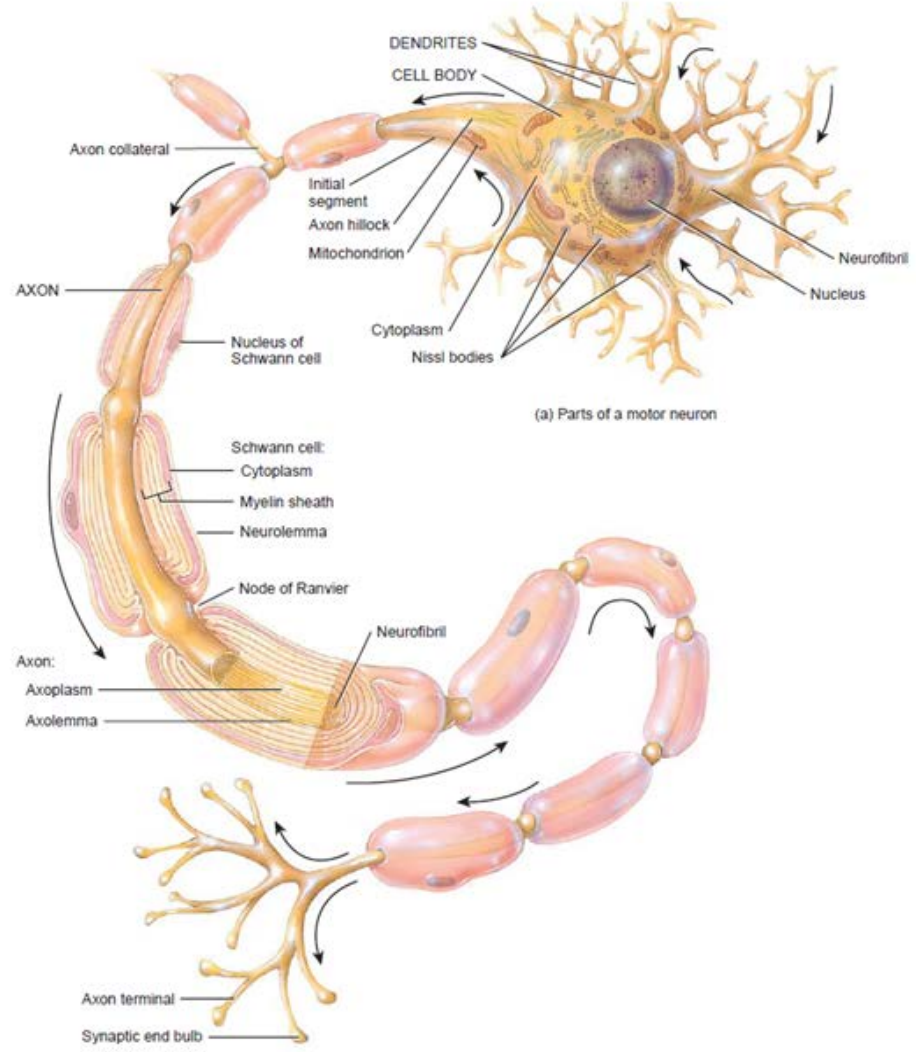

Figure 2. Structure of a multipolar neuron - a neuron with a large cell body, several short dendrites, and a single long axon. Arrows indicate the direction of information flow:dendrites --> cell body --> axon --> axon terminals [7]

Neuroglia of the PNS completely surround axons and cell bodies and are grouped into two cell types: Schwann cells and satellite cells. Schwann cells encase the larger axons of peripheral neurons in lipid-rich sheaths formed by tightly wound layers of cell membrane [Figure 3]. The layers are composed of a myelin sheath, which has a higher proportion of lipid than other surface membranes. Axons that have myelin sheaths are called myelinated axons, and those that lack these sheaths are called unmyelintaed axons. The myelin sheath electrically insulates the axon of a neuron and increases the speed of nerve impulse conduction. 


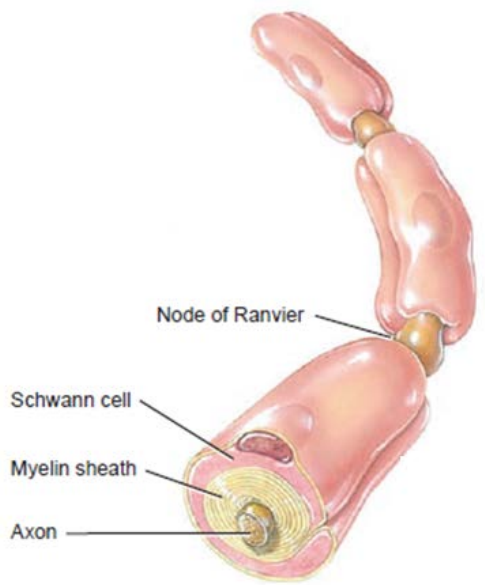

Figure 3. Schwann cells of the PNS completely surround axons and cell bodies of neurons [7]

Scwann cells are responsible for the production of myelin sheaths in the PNS, beginning during fetal development. Each Schwann cell wraps about 1 millimeter of a single axon's length by spiraling many times around the axon [Figure 4]. Up to 100 layers of Schwann cell membrane becomes the myelin sheath, with the Schwann cell's cytoplasm and nucleus forming the outermost layer, the neurolemma. The neurolemma aids regeneration when an axon is injured by forming a regeneration tube that guides and stimulates regrowth of the axon. Narrow gaps in the myelin sheath between Scwann cells are called nodes of Ranvier [Figure 2]. The nodes of Ranvier play a key role during the propagation of action potentials.

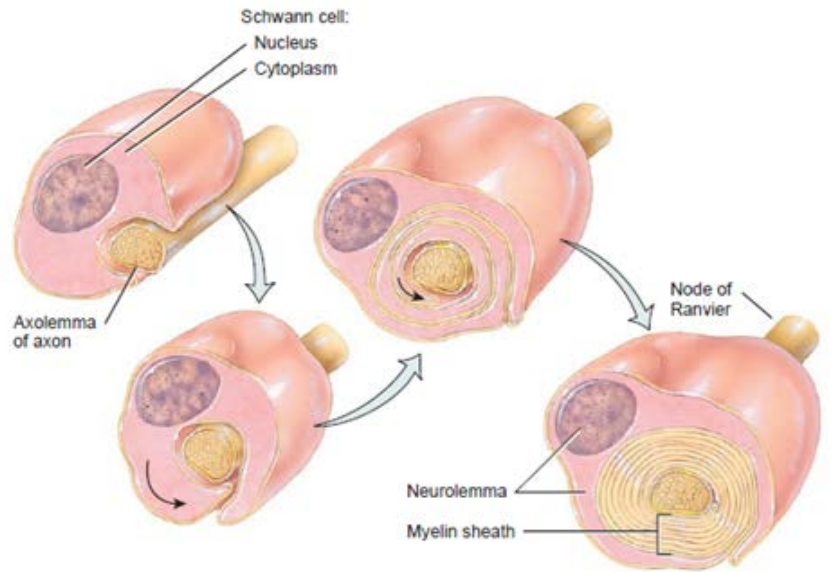

Figure 4. Transverse sections of stages in the formation of a myelin sheath [7] 


\section{NERVE COMMUNICATION}

Neurons communicate with one another using two types of electrical signals: Graded potentials for short-distance communication and action potentials for long-distance communication. The production of graded potentials and action potentials depends on the presence of specific types of ion channels on the plasma membrane that open and close in response to specific stimuli, and on the existence of a membrane potential which is an electrical potential difference (voltage) across the membrane.

Potassium ions $\left(\mathrm{K}^{+}\right)$are the major intracellular positive ion, while sodium ions $\left(\mathrm{Na}^{+}\right)$are the major extracellular positive ion. The distribution is created largely by the sodium-potassium pump, which actively transports sodium ions out of the cell and potassium ions into the cell. The distribution of ions is also affected by channels in the cell membrane that determine membrane permeability. In the case of a resting neuron, the membrane potential is called the resting membrane potential and has a typical value of $-70 \mathrm{mV}$. The negative sign indicates the excess negative charges on the inside of the cell membrane [Figure 5]. Sodium and Potassium ions continue to diffuse across the cell membrane at a rate of three sodium ions leaking into the cell for every two potassium ions that leak out, which is then balanced by the sodium-potassium pump.

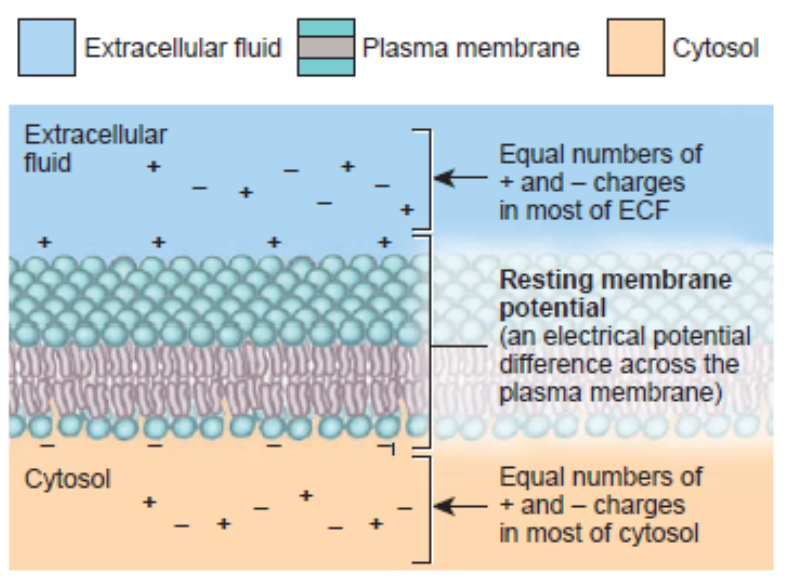

Figure 5. Distribution of charges that produce the resting membrane potential of a neuron [7] 
An action potential is a sequence of rapidly occurring events that decrease and reverse the membrane potential and then eventually restore it to the resting state. The phases that occur during an action potential include the: depolarization phase, repolarization phase, afterhyperpolarization phase, and refractory period. In order for depolarization to occur, a threshold stimulus must occur to bring the membrane potential to a threshold value (-55 $\mathrm{mV}$ in many neurons). Once the threshold value is reached, the depolarization phase begins where voltagegated sodium channels open rapidly and cause an inrush of sodium ions. The inflow of sodium ions changes the membrane potential to $+30 \mathrm{mV}$, meaning the inside of the membrane is $30 \mathrm{mV}$ more positive than the outside. These voltage-gated sodium channels close quickly, but almost simultaneously, slower voltage-gated potassium channels open and briefly increase potassium permeability. The slowing of sodium inflow and the acceleration of potassium outflow causes the repolarization phase, which causes the inside membrane potential to change from $+30 \mathrm{mV}$ to -70 $\mathrm{mV}$. While the voltage-gated potassium channels are open, outflow of potassium may be large enough to cause an after-hyperpolarization phase of the action potential, where the membrane potential may become as negative as $-90 \mathrm{mV}$. As the voltage-gated potassium channels close, the membrane potential returns to the resting level of $-70 \mathrm{mV}$ and remains in the resting state until it is stimulated again.

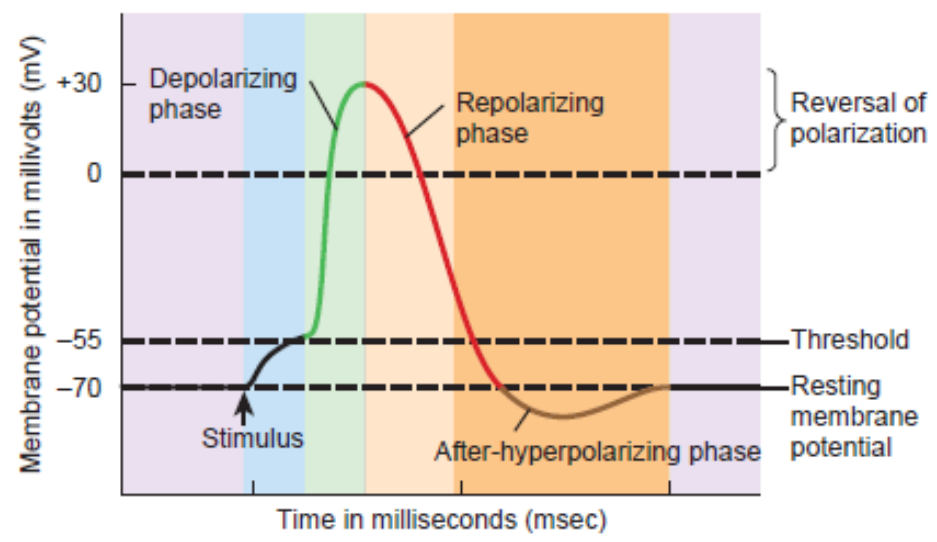

Key:

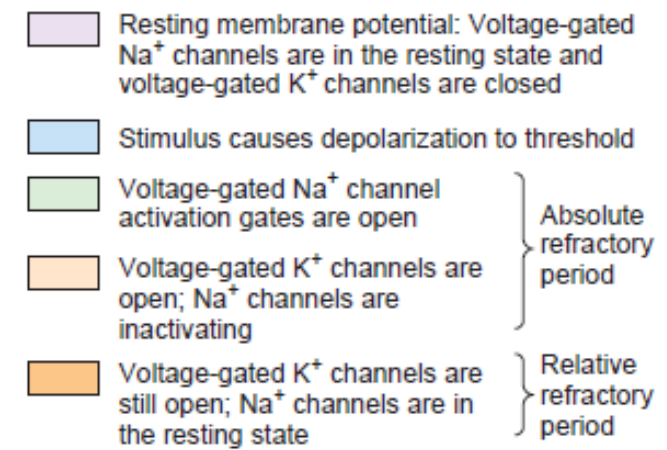

Figure 6. Main phases of an action potential [7] 
Three major factors that affect the speed of propagation of an action potential include: amount of myelination, axon diameter, and temperature. Action potentials travel, or propagate, more rapidly along myelinated axons than along unmyelniated axons, due to saltatory conduction. Saltatory conduction is when action potentials only occur at the nodes of Ranvier, and therefore appear to "leap” between long segments of myelinated axolemma. Jumping from node to node makes current flow much faster than in an unmyelinated axon of the same diameter. Saltatory conduction also represents a more energy-efficient mode of conduction since less ATP is used by sodium-potassium pumps to maintain the intracellular and extracellular balance of ions. The diameter of the axon also affects the speed of nerve impulse conduction - larger diameter axons propagate action potentials faster than smaller ones. This is because larger diameter axons have a greater total volume for charges to flow through and correspondingly meet less internal resistance from the membrane. Temperature also affects the speed of propagation - higher temperatures result in higher propagation speeds [7].

\section{CLASSIFICATION OF NERVE FIBERS}

Axons can be classified into three major groups based on the amount of myelination, the diameter, and the propagation speeds. Table 1 shows the various types of fibers and some of their characteristics. 
Table 1. Nerve Fiber Types and Characteristics [7]

\begin{tabular}{|c|c|c|c|}
\hline Fiber Type & Diameter Size $(\mu \mathrm{m})$ & Propagation Speed & Function \\
\hline $\begin{array}{l}\text { A Fibers (Myelinated) } \\
\qquad \begin{aligned} & \\
\bullet & \mathrm{A} \alpha \\
\bullet & \mathrm{A} \gamma \\
\bullet & \mathrm{A} \delta\end{aligned}\end{array}$ & $5-20$ & $\begin{array}{l}12-130 \mathrm{~m} / \mathrm{sec} \\
(27-280 \mathrm{mi} / \mathrm{hr})\end{array}$ & $\begin{array}{l}\text { Sensory neurons that } \\
\text { propagate impulses } \\
\text { associated with touch, } \\
\text { pressure, position of } \\
\text { joints, and some } \\
\text { thermal and pain } \\
\text { sensation }\end{array}$ \\
\hline B Fibers (Myelinated) & $2-3$ & $\begin{array}{l}<15 \mathrm{~m} / \mathrm{sec} \\
(<32 \mathrm{mi} / \mathrm{hr})\end{array}$ & $\begin{array}{l}\text { Conduct sensory } \\
\text { nerve impulses from } \\
\text { the viscera to the } \\
\text { brain and spinal cord }\end{array}$ \\
\hline $\begin{array}{c}\text { C Fibers } \\
\text { (Unmyelinated) }\end{array}$ & $0.5-1.5$ & $\begin{array}{l}0.5-2 \mathrm{~m} / \mathrm{sec} \\
(1-4 \mathrm{mi} / \mathrm{hr})\end{array}$ & $\begin{array}{c}\text { Conduct some } \\
\text { sensory impulses for } \\
\text { pain, touch, pressure, } \\
\text { heat and cold from } \\
\text { the skin, and pain } \\
\text { impulses. }\end{array}$ \\
\hline
\end{tabular}

These nerve fiber types can then be further classified according to size into large, medium, or small fibers, which also correlates with the degree of myelination. Large nerve fibers are heavily myelinated and include A-alpha fibers and A-beta fibers. Medium-sized fibers are known as A-gamma fibers. Small fibers include myelinated A-delta fibers and unmyelinated Cfibers [1].

\section{NERVE DAMAGE}

\section{DAMAGE AND REPAIR}

Injury to the cell body usually kills a mature neuron, however, a damaged peripheral axon may regenerate depending on the damage. Axons and dendrites that are associated with a neurolemma may undergo repair if the cell body is intact, if the Schwann cells are functional, and 
if scar tissue formation does not occur too rapidly $[6,7]$. When there is injury to an axon or a disease process, changes will occur in the cell body and in the portion of the axon distal to the site of injury. By the third to fifth day, the part of the axon distal to the damaged region and the myelin sheath deteriorate. This debris is phagocytosed by macrophages. A regeneration tube may be formed across the injured area by two Schwann cells joining on either side of the injured site. This tube guides growth of a new axon across the injured area toward the distally located receptors and effectors. Thus, some sensory and motor connections are reestablished and some functions restored. Eventually, remaining Schwann cells proliferate along the length of the degenerating portion and form new myelin around the growing axon.

\section{PERIPHERAL NEUROPATHY}

Peripheral neuropathy describes damage to the peripheral nervous system, which damages vital connections between various parts of the body and the brain and spinal cord. Peripheral neuropathy may be caused by physical trauma to nerve, autoimmune responses, systemic disease, or genetic errors. Different clinical conditions are knows to selectively impact various size fibers. Peripheral neuropathy can be categorized based on the function of the involved nerve fibers or on their diameter and conduction velocity.

Small fiber dysfunction can be defined as a generalized peripheral neuropathy in which the small diameter myelinated and unmyelinated nerve fibers are affected, either exclusively or to a much greater degree than the large diameter myelinated fibers [8]. Small fiber neuropathy (SFN) has been associated with many medical conditions including glucose dysmetabolism, connective tissue disease, HIV infection, hereditary diseases, alcoholism, and others [1]. Additional underlying diseases are shown in Table 2. Nerve conduction studies and electromyography, which are primarily used for the evaluation of large fiber neuropathies, are generally normal diagnostic procedures for patients with SFN as well. However, it is difficult to diagnose SFN since, in many patients, the results of nerve conduction studies and EMG are 
normal. The normal results cause SFN to be easily overlooked or misdiagnosed [1]. These tests are often performed anyway in order to rule out subclinical involvement of large fibers. Although there are diagnostic techniques available, they are not widely available; therefore it has been difficult to establish a gold standard for the evaluation of SFN. 
Table 2. (Idiopathic) Causes of small fiber neuropathy [9]

\begin{tabular}{|c|c|}
\hline \multirow{2}{*}{ Inherited } & Familial Amyloidosis \\
\hline & $\begin{array}{l}\text { Autosomal recessive hereditary } \\
\text { neuropathy }\end{array}$ \\
\hline & Fabry’s disease \\
\hline & $\begin{array}{l}\text { Heriditary sensory and autonomic } \\
\text { neuropathy }\end{array}$ \\
\hline & Ross syndrome \\
\hline & Friedreich’s ataxia \\
\hline & Tangier disease \\
\hline \multirow[t]{16}{*}{ Acquired } & Diabetes mellitus \\
\hline & Impaired glucose tolerance \\
\hline & Alcoholism \\
\hline & Systemic amyloidosis \\
\hline & Vasculitis \\
\hline & Sarcoidosis \\
\hline & Sjögren’s disease \\
\hline & Systemic lupus erythematosus \\
\hline & Guillain-Barre syndrome \\
\hline & Antecedent viral infection \\
\hline & HIV \\
\hline & Antisulfatide antibodies \\
\hline & Hyperlipidemia \\
\hline & Complex regional pain syndrome \\
\hline & Paraneoplastic syndrome \\
\hline & Neurotoxic medication \\
\hline
\end{tabular}


Chronic inflammatory demyelinating polyneuropathy (CIDP) is a neurological disorder characterized by progressive weakness and impaired sensory function in the legs and arms. It is an example of a large fiber peripheral neuropathy since it is caused by damage to the myelin sheath of the large peripheral nerves. CIDP is closely related to Guillain-Barre syndrome and it is considered the chronic counterpart of that acute disease. Electrodiagnostic testing is recommended for all patients with suspected CIDP. Additional diagnostic evaluations include cerebrospinal fluid analysis, nerve biopsy, Magnetic Resonance Imaging (MRI) of spinal roots, laboratory studies, and evaluation for inherited neuropathies. While these diagnostic evaluations can be helpful to the clinician, none have ideal sensitivity and specificity.

\section{DIAGNOSTIC TECHNIQUES}

\section{LABORATORY EXAMINATIONS}

Most experts recommend cerebrospinal fluid analysis in order to demonstrate the typical findings in several peripheral neuropathies: increased protein and a normal or only slightly elevated cell count [10]. More extended laboratory testing may also be necessary in some patients to search for causes of demyelinating polyneuroptahy, as well as concurrent diseases.

\section{NERVE BIOPSY}

The diagnostic value of nerve biopsy has been extensively debated, with some experts believing that there is no diagnostic value, whereas others view it as essential for diagnosis and management in up to 60 percent of patients with chronic demyelinating polyneuropathy [10]. Nerve biopsies have a low diagnostic yield in chronic inflammatory demyelinating polyneuropathy, for several reasons. The most prominent abnormalities may lie in the proximal segments of the nerves or roots or in motor nerves, which are areas not accessible to biopsy. Also, secondary axonal changes starting early in the disease processes may over-shadow the initial signs of demyelination and inflammation by the time a biopsy is performed. 
MRI may be used to demonstrate gadolinium enhancement and enlargement of proximal nerves or roots, reflecting active inflammation and demyelination in the caudal equine or brachial plexus [10]. Abnormalities of the brachial plexus with irregular swelling and increased signal intensity were detected in about 50 percent of patients with CIDP.

\section{CONTACT HEAT-EVOKED POTENTIALS}

Contact heat-evoked potentials (CHEP) is a non-invasive assessment to study the function of painful and thermal pathways in patients with diabetic neuropathy and healthy adults. This method could potentially evoke fast pain at the cutaneous nocireceptors and be used to mediate nocireceptive pathway of small diameter, myelinated A-fibers [11]. Research suggests that CHEPs could be utilized as an objective and non-invasive tool to detect small myelinated fiber damage in Guillain-Barré syndrome (GBS) patients.

\section{NERVE CONDUCTION VELOCITY}

The mean nerve conduction velocity (NCV) has long been a clinically valuable indicator in the diagnosis and assessment of neuromuscular disorders. The estimation of the average conduction velocity of peripheral nerves has been widely used in clinical neurology and traumatology. It has also been added to diagnostic tests of several metabolic disorders, since the myelin sheath is closely related to the propagation velocity. During a NCV test, a measurement is taken of the speed of conduction of a compound evoked potential along a nerve [12]. The measurement is taken from surface electrode patches placed on the surface of the skin to stimulate a specified nerve. An example setup for a NCV test is shown in Figure 7. One electrode stimulates the nerve while the other records when the action potential reaches its position. With this information, it can be determined how fast or slow the nerve is conducting by using the time it takes for the signal to move from the stimulating electrode to the recording electrode and the distance between the two electrodes [12]. 


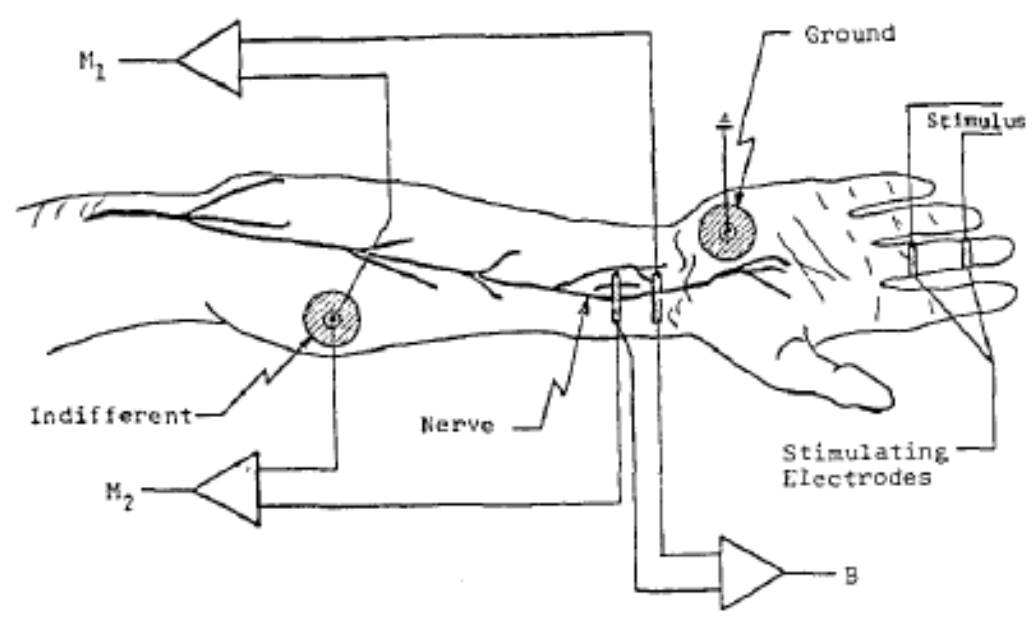

Figure 7: Electrode configuration for nerve conduction velocity test [13]

NCV distribution estimation attempts to establish the relative number of active fibers per group of fibers. NCV tests have the potential of providing information to help assess peripheral neuropathies, but NCV tests do not provide detailed information regarding the characteristics of individual nerve fibers that contribute to the compound evoked potential. This is due to the faster conducting fibers dominating the accumulated signal that the NCV test measures. The measurement found in a NCV test is essentially created from an average of contributions made by all of the individual nerve fibers within a nerve trunk rather than investigating the individual nerve fibers themselves, so a precise diagnosis cannot always be determined solely from this test [12]. Therefore, a more useful diagnosis for a clinician would be to use a measurement technique that could extract information about the population of nerve fibers within the nerve trunk that are contributing to the evoked potential. Determining this size distribution of nerve fibers could help differentiate between various peripheral neuropathies that are shown to impact specific fiber size. 
Szlavik et al. [3] presented a novel technique for estimating the size distribution of contributing nerve fibers that is linearly related to the conduction velocity distribution. The technique is based on an estimation of the group delay between two sets of recording electrodes associated with the individual fibers that contribute to a maximal compound-evoked potential. The group delay information is then used to estimate the diameters of the activated fibers as well as the propagation delays of individual single fiber-evoked potentials to a reference electrode. This process allows for reconstruction of an estimated maximal compound evoked potential, from the individual single-fiber-evoked potentials, at the first recording site. The physical setup is shown in Figure 8, where a stimulator is used to excite a subcutaneous nerve trunk consisting of a group of electrically independent nerve fibers.

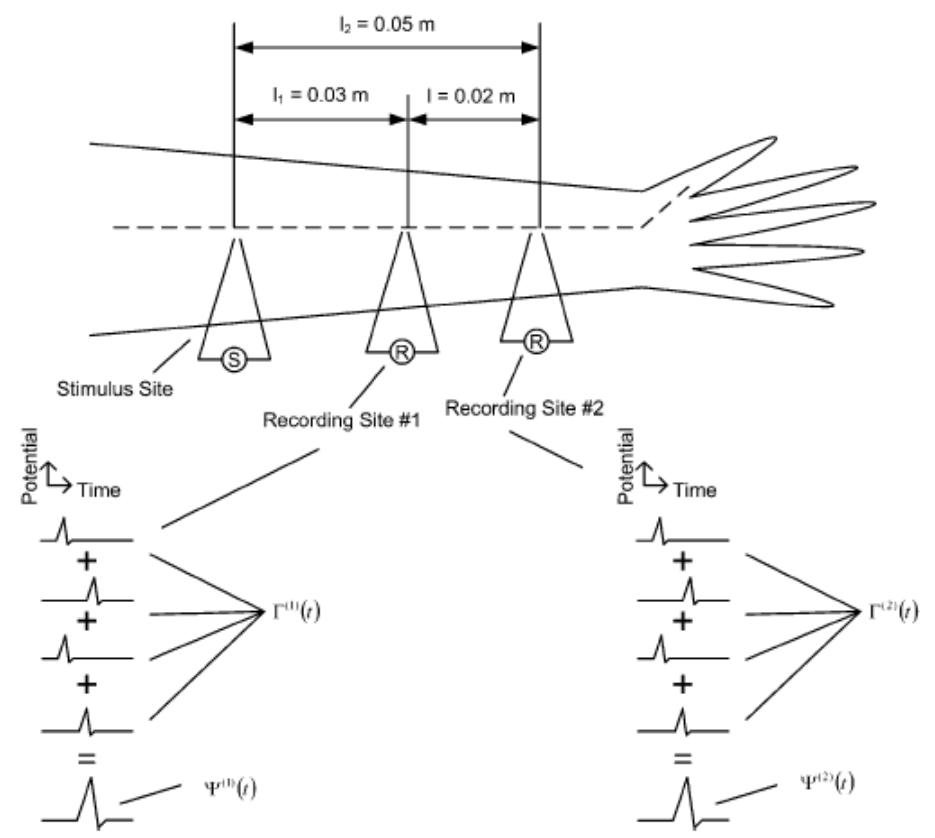

Figure 8: Physical configuration for the method proposed by Szlavik et al [3]

Using a series of successively increasing current stimulus pulses, the successively recorded compound evoked potentials can be decomposed into their constituent single fiber action potentials in a manner analogous to the protocol used in the McComas et al. motor unit 
number estimation technique $[3,14]$. The results of the simulation study presented by Szlavik et al. demonstrate that the technique presented can, with reasonable accuracy, retrieve the conduction velocity distribution in the presence of noise introduced through variations in the perpendicular distance between the recording site and the contributing fiber. The complete methodology and results are shown in the article by Szlavik et al. [3].

\section{EXPERIMENTAL MODEL}

The rat animal model was chosen for this study to follow the Institutional Animal Care and Use Committee (IACUC) regulations on choosing the lowest-order animal model allowable. The rat was chosen due to the following reasons [15]:

- The rat is neurologically comparable to humans both anatomically and physiologically.

- The rat is of relatively small size and easily handled.

- The space around the sciatic nerve allows for ease of electrode placement.

- The rat's large sciatic nerve makes it simple to obtain a sufficient distribution of nerve fiber diameters.

The surgical protocol outlined by Sammy Nicolas [15] was followed to stimulate and extract the rat sciatic nerves used in this experiment and can be found in the dissertation: Implementation of Novel Group Delay Decomposition Method and Surgical Protocol for Assessing Peripheral Neuropathy [15]. 


\section{CHAPTER III: METHODS}

\section{INTRO DUCTION TO HISTOLOGY AND H\&E STAINING}

Histology is the science that deals with the study of tissues, and is commonly performed by examining cells and tissues by sectioning and staining [7]. Elaborate steps are taken to prepare tissue for microscopic viewing. The specimen must be fixed (preserved) and then cut into sections (slices) thin enough to transmit light or electrons. Finally the specimen must be stained in order to enhance contrast. Many dyes consist of negatively or positively charged molecules (acidic and basic stains respectively) that bind within the tissue to macrcomolecules of the opposite charge [16]. Because different parts of the cells and tissues take up different dyes, the stains distinguish different anatomical structures. Hematoxylin and Eosin (H\&E) staining is one of the most common stains in histology and has been unchanged for many years because it works well with a variety of fixatives and displays a broad range of cytoplasmic, nuclear, and extracellular matrix features. Hematoxylin has a deep blue-purple color and stains nucleic acids by a complex, incompletely understood reaction. Eosin is pink and stains proteins nonspecifically. In a typical tissue, nuclei are stained blue, whereas the cytoplasm and extracellular matrix have varying degrees of pink staining. This method was chosen due to its simplicity and reliability for staining the nucleus and cytoplasm of tissue and for visualizing results. The histology and staining process begins with tissue extraction, tissue fixation, followed by microtomy (sectioning), depariffinization and rehydration, staining, and microscopy for image analysis.

\section{TISSUE EXTRACTION}

The procedure for extracting the rat sciatic nerves used in this experiment was outlined in the surgical protocol written by Sammy Nicolas [15]. The dissertation outlines a surgical procedure for exposing and stimulating the sciatic nerve of an anesthetized rodent for purposes of 
obtaining conduction velocity readings. This method was chosen to impose the least amount of physical trauma to the exposed nerve, in order to obtain accurate results. The nerves were previously labeled, extracted and placed in formaldehyde to preserve the tissue for future analysis.

\section{TISSUE FIXATION}

With the rat sciatic nerves extracted, it was necessary to embed the nerves in a paraffin wax block in order to support cellular components adequately during microtomy. Embedding is the casting or blocking of tissue section, which involves the enclosure of the tissue in the infiltration medium used for processing, and then allowing the medium to solidify. Paraffin wax embedding was selected due to the elasticity and density of the medium, allowing for the best tissue-medium adhesion. The tissue was embedded by placing it in an upright position in a metal mold and allowing molten paraffin wax to encase the tissue. Correct orientation was a crucial step in embedding the tissue, since incorrect placement and orientation of the tissue could result in diagnostically important tissue elements being missed or damaged during microtomy. Tubular tissues and walled specimens, such as the rat sciatic nerve, should be embedded so as to provide transverse sections showing all tissue layers. The vertically placed rat sciatic nerve was then placed on a cold block to solidify the paraffin wax encasing. The paraffin wax embedding process was repeated for three rat sciatic nerves, leaving three paraffin wax blocks labeled: 12-028 (control), 12-029 (experimental), and 12-030 (experimental). The three blocks were then stored for later analysis.

\section{$\underline{\text { MICROTOMY }}$}

Once the tissue sections were embedded in the paraffin wax blocks, the samples would need to be sectioned to produce cross sections of the tissues for staining, imaging, and analysis. Prior to sectioning, samples were incubated at $4^{\circ} \mathrm{C}$ for 15 minutes to facilitate tissue sectioning and to prevent premature melting of the paraffin wax during the sectioning process. While the 
samples were incubating, the tissue warm water bath was filled halfway and plugged into an outlet, the tissue oven was set to $60^{\circ} \mathrm{C}$, and the microtome was set up for sectioning by placing a microtome blade in place. Once the samples had been incubated for 15 minutes, each block was placed on the tissue holder of the microtome and clamped securely. With Latex gloves, the microtome blade was placed and locked into position and the block was lowered to the level of the blade by unlocking and turning the hand wheel. The mounted blade was then moved towards the sample until it was flush against the block and locked in place. Since the actual tissue is embedded a few millimeters (mm) within the paraffin wax block, the initial section thickness was set to 15 microns $(\mu \mathrm{m})$ and sections were taken until the tubular tissue inside the wax was exposed to the blade.

Initially, the microtome was adjusted to $6 \mu \mathrm{m}$ and it was attempted to section the sample. Subsequent slices at $6 \mu \mathrm{m}$ were being split upon slicing and sections were not able to be obtained. This could be due to the section thickness being too thin, the microtome blade being too dull or damaged, or the paraffin wax blocks not being cold enough. The wax block was placed in the freezer once again for incubation for an additional 15 minutes, for a total of approximately 30 minutes. After the incubation period, the blocks were removed from the freezer and steps were repeated to obtain sections at a thickness of $6 \mu \mathrm{m}$. Once again the sections were being split upon slicing, so the thickness of the slices was increased. The microtome was then adjusted to $7 \mu \mathrm{m}$ and subsequent slices were taken. After many attempts, clean, undamaged $7 \mu \mathrm{m}$ sections were produced and were carefully placed on the water surface of the warm water bath using forceps. For this step, it was important to place the sections into the water without any wrinkles, in order to facilitate the transfer from the water bath to the glass slides. Once in the water bath, adjacent strips of slices were separated to create sets of one or two sections per glass slide. To do this, forceps were used to poke the connected edges between two slices until the sections separated. The unused tissue was discarded, while the usable tissue was mounted onto a glass slide by 
dipping the slide into the water bath at a $45^{\circ}$ angle and allowing for the section to smoothly sit onto the glass slide, using forceps as a guide. During this step it was important for there not to be any air pockets, bubbles, or wrinkles in the section, in order to prevent any tissue damage during the deparaffinization, rehydration, or staining process. The slides were then placed into the microscope slide rack and allowed to dry for about 15 minutes. This procedure was followed to create multiple slides from each of the paraffin wax embedded samples. Each sample block created five slides, totaling 15 slides, with either one or two sections on each slide for the staining and imaging procedures.

\section{DEPARAFFINIZATION AND REHYDRATION}

Deparaffinization and rehydration are crucial steps in preventing poor staining of the specimen due to incomplete removal of paraffin wax or inadequate penetration of stains into the tissue. To begin this process, the slides were set in a staining rack and then placed into the slide oven, now at $60^{\circ} \mathrm{C}$, for about 30 minutes, or until the paraffin wax melted and became clear. While the samples were in the oven, the following rehydration washes were set up under the fume hood: two xylene (Xylene, Richard-Allen Scientific) baths, two 100\% ethanol (Sigma-Aldrich Ethanol) baths, one 95\% ethanol bath, and one distilled water bath. The H \& E staining washes were also set up under the fume hood during this time and included: one hematoxylin bath (Hematoxylin 7211, Richard-Allen Scientific), one distilled water bath, one bluing bath, one clarifier bath, and one Eosin bath (Eosin-Y, Richard-Allen Scientific). The washes were set up in glass trays, ensuring that enough liquid was poured in to cover the entire slide once the staining rack was dipped into the tray. Once the paraffin wax was melted, the staining rack was removed from the oven and allowed to cool for approximately five minutes. The following steps were taken to continue the deparaffinization and rehydration process: staining rack was placed in the first xylene bath for three minutes, transferred to the other xylene bath for three minutes, and then placed in the xylene bath for an additional three minutes. The staining rack was then placed in a 
$100 \%$ EtOH bath for two minutes, transferred to the other $100 \%$ EtOH bath for another two minutes, and then placed in the 95\% EtOH bath for two minutes. The staining rack was then allowed to sit in the distilled water bath.

\section{H\&E STAINING}

Once the deparaffinization and rehydration process had been completed, the samples were ready for the $\mathrm{H} \& \mathrm{E}$ Staining procedure. From the distilled water bath, the slide rack was placed into the Hematoxylin bath for four minutes. The rack was then transferred to a distilled water bath for one minute, then to the clarifier bath for 30 seconds, and back to the distilled water for one minute. From the distilled water, the staining rack was placed in the bluing solution for one minute, and then placed back in the distilled water for another minute. The staining rack was then placed in the 95\% EtOH bath for one minute, then into the Eosin bath for one minute and 30 seconds. The staining rack was then submerged in 100\% EtOH for one minute, for three repetitions. Lastly, the rack was placed in xylene for three minutes, for three repetitions, and then allowed to air dry. The Sectioning and Staining protocol that was followed during this study is included in Appendix D. Figure 9 shows the set up for the rehydration and $\mathrm{H}$ \& E staining procedures under the fume hood. 


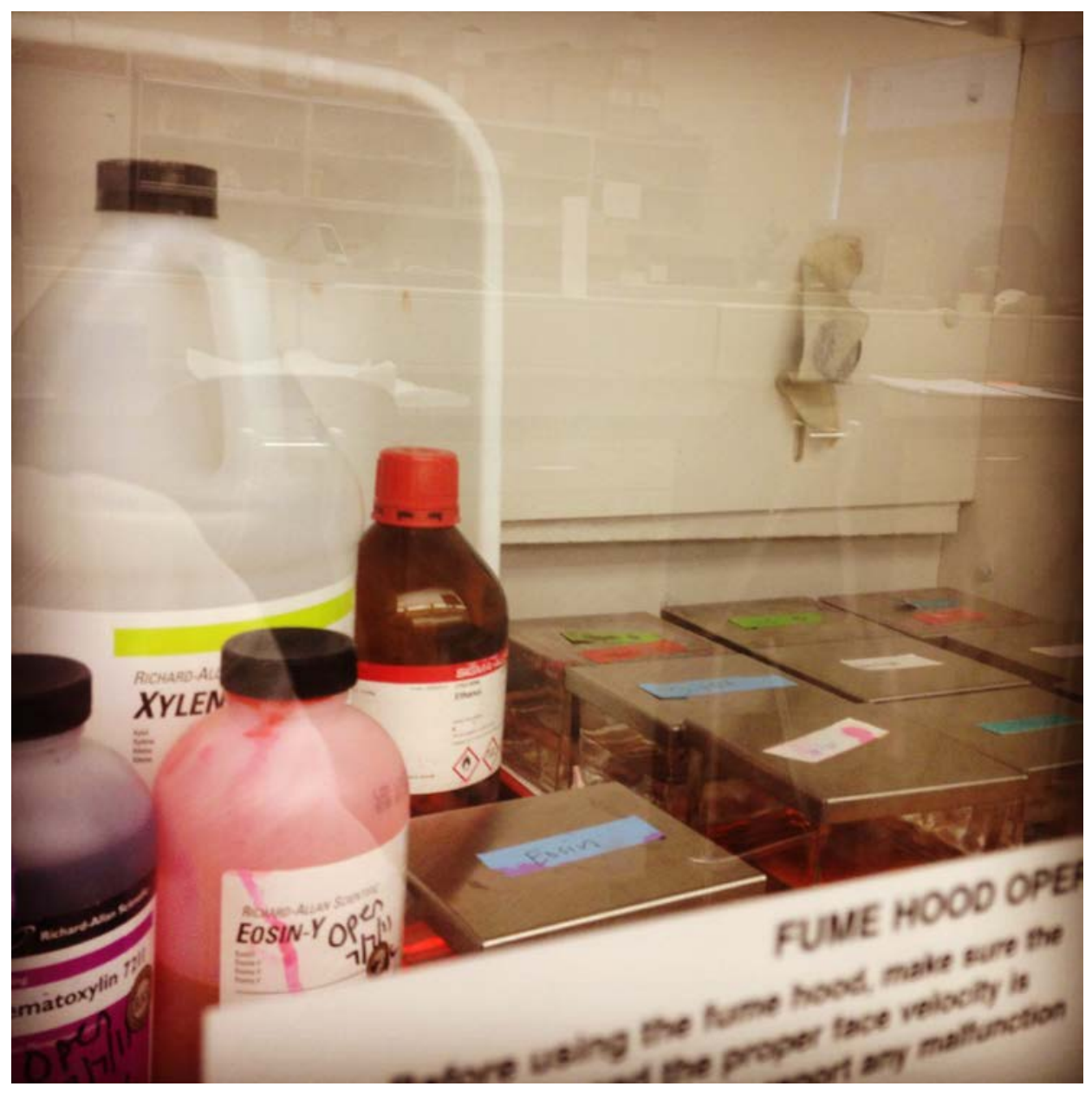

Figure 9: View of the $H \&$ E staining procedure set-up under the fume hood.

It is crucial that during the deparaffinization, rehydration, and staining steps, fresh fluid baths are prepared before each trial to enhance the effectiveness of the stains. During an initial trial, fluid washes were reused from a previous experiment which led to undesirable results. After going through the entire staining procedure, the slides were examined under a microscope and unusable images were produced. Figure 10 shows an example of the microscopy results after old, recycled washes were used. 


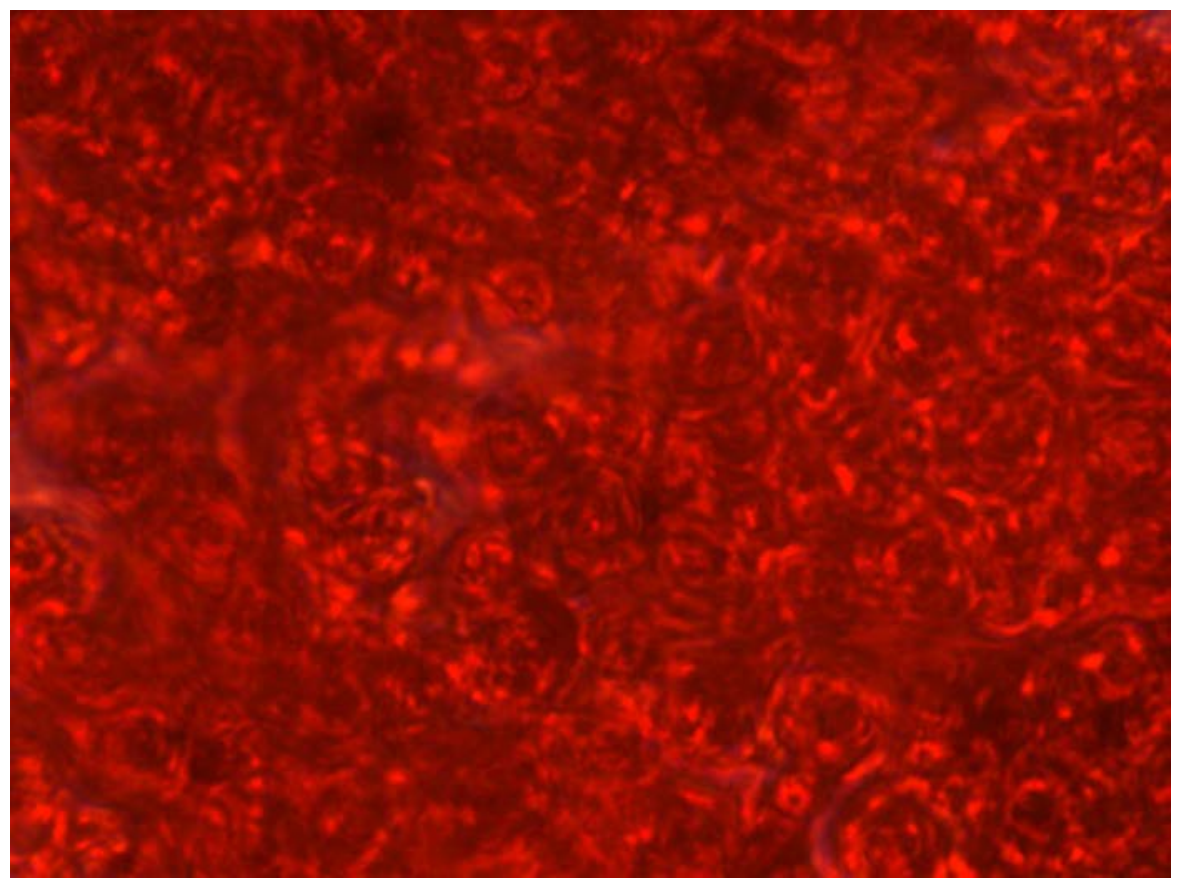

Figure 10: Brightfield image of an unusable $H \&$ E stained rat sciatic nerve sample using a 100x objective

An explanation for Figure 10 is that the hematoxyolin stain did not penetrate into the cells, due to an error during the staining process. The hematoxylin stain should have stained the cell nuclei blue, but in this case, only the red Eosin stain can be seen. After additional similar images had been captured during microscopy analysis, it was concluded that there had been an error in the staining process, and it was to be repeated at a later time. On the next staining day, all baths were prepared on the same day as when the staining procedure was completed. The same steps as previously mentioned were repeated using the fresh fluid washes. The following steps were then taken to produce quantifiable images of the stained samples.

\section{MICROSCOPY}

Once all the slides were stained following the H\&E procedure, an Olympus Laboratory Microscope (BX41, Olympus America Inc., Melville, NY) was used to visualize the results of the H\&E staining process using bright field microscopy. The 100X objective (Olympus, 100x, 1.25 oil, $\infty /-)$ was placed on the microscope in order to achieve the correct magnification to see the 
results of the staining process. It was necessary to have an objective that could clearly see the stained nuclei and cell membrane of the corresponding tissue sample. The microscope was turned on through a series of steps and then calibrated through the live preview shown in the Q Capture Pro 6.0 software installed on the adjacent computer. First, the main power supply for the microscope and the power switch for the Q-Imaging Camera (Retiga EXi FAST 1394 digital CCD camera) installed on the microscope were turned on. Then, the lenses were switched to FW2, where the number six was selected in order to avoid a colored filter (used during fluorescent microscopy). Once the camera and microscope were turned on, the slides were prepared for viewing. Since the $100 \mathrm{X}$ objective required oil as the immersion fluid, a drop of immersion oil was placed onto the slide before locking the slide into the microscope stage. The objective was moved towards the slide using the focus knobs, until it touched the immersion oil, and the samples were located in the eyepiece by adjusting the focus knobs and by moving the stage. Once clear images of the samples were located in the eyepiece, images were sent to the Q Capture Pro screen by pulling out the pin located near the camera, allowing for the light to enter the camera instead of the eyepiece. Images were then captured using the Q-Imaging Camera and saved using Q Capture Pro 6.0. An example image of a correctly stained sample is shown in Figure 11. Clear images were saved for future analysis: nine images for block 12-028, six for block 12-029, and seven for block 12-030. 


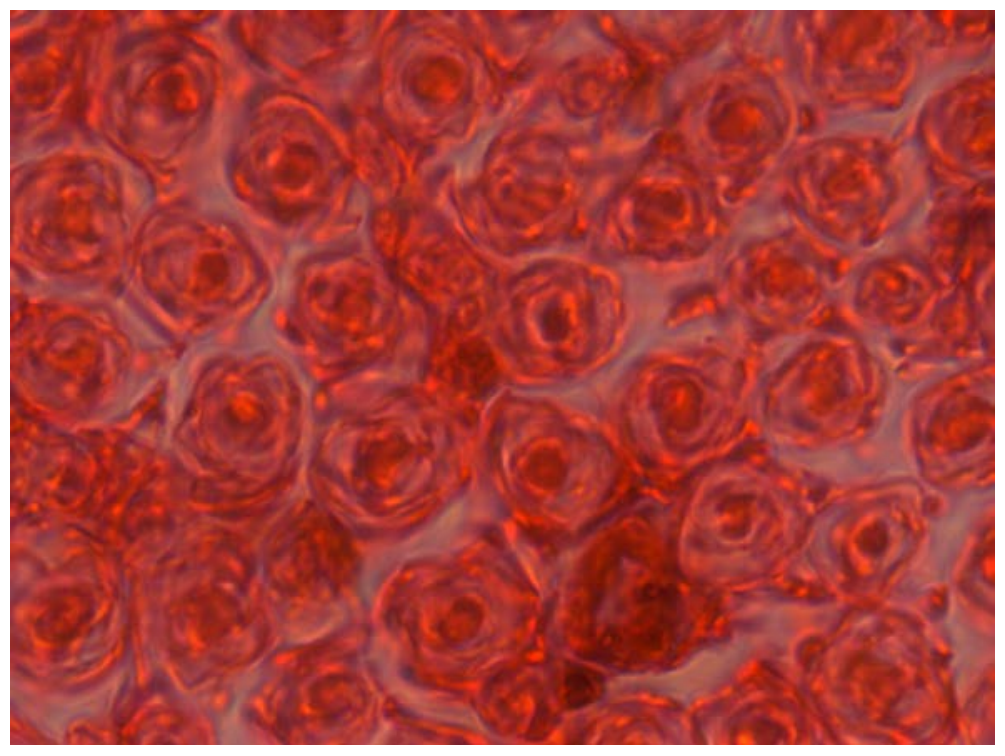

Figure 11: H\&E stained image taken using brightfield microscopy with a 100X objective

\section{IMAGE ANALYSIS}

The images were analyzed using Image $\mathrm{J}$, a Java-based image processing program commonly used for biological image analysis. This software allows for analysis of images, cell count, comparison of fiber diameters, and other measurements for each tissue sample. The primary purpose of imaging the sciatic nerve was to measure the fiber diameters. Image $\mathrm{J}$ allows the user to take measurements in an image using a line tool. After using the line tool, Image $\mathrm{J}$ outputs the measurement in units of pixels. Since pixel is not a useful unit to measure lengths in biologics, conversion from pixels to micrometers was necessary. This is easily achieved using a conversion tool in the Image $\mathrm{J}$ program. The conversion tool requires the user to input a known length (micrometer) of a ruler in the given image and the length in pixels of the same ruler. Measurements taken after calibration using the line tool will output lengths with the units of micrometers. In this case, the known length was the Edmund Optics Stage Micrometer (36121, 1mm/100Divisions) which was used to set the correct unit scale. Figure 12 shows the Edmund Optics Stage Micrometer. Detailed instructions on how to measure lengths in Image $\mathrm{J}$ is given in Appendix A. 


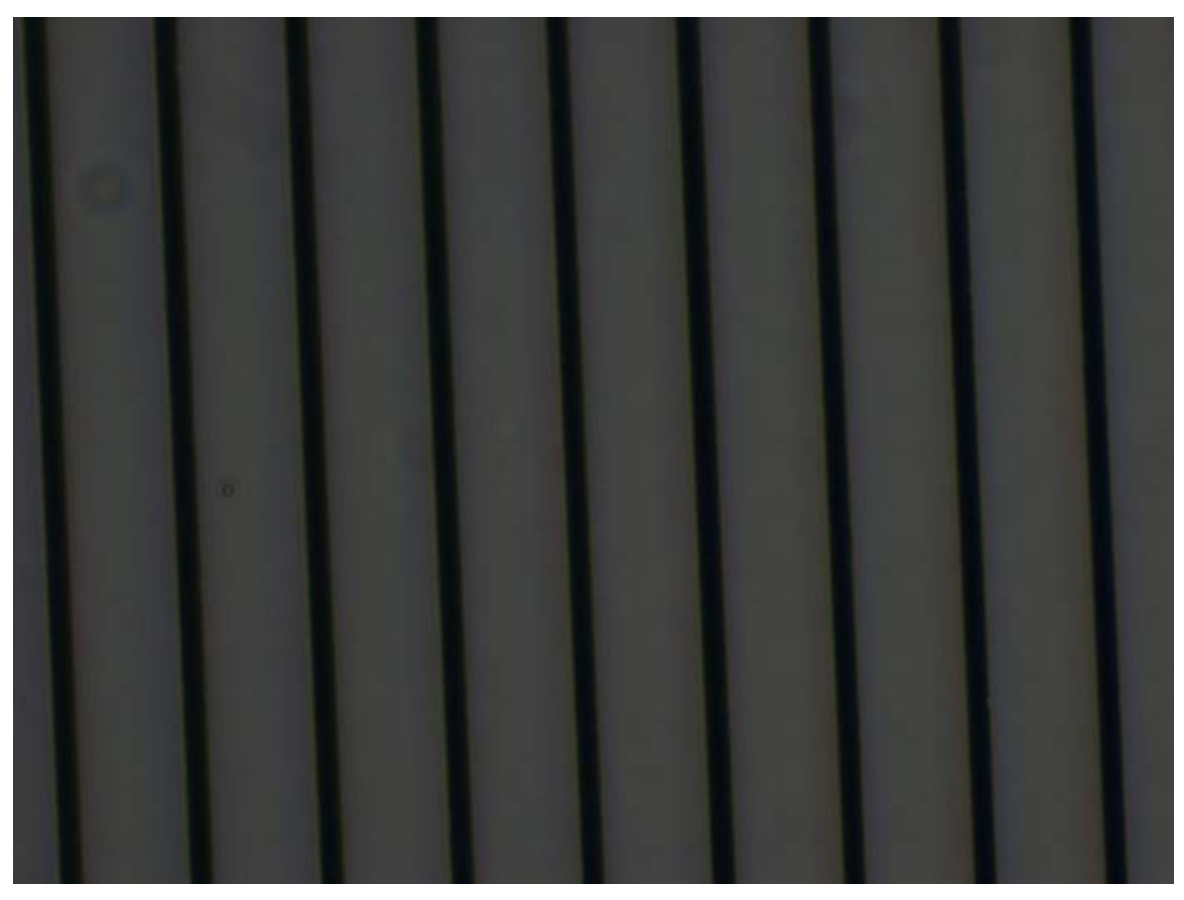

Figure 12: Edmund Optics Stage Micrometer 1mm/100Divisions

\section{FIBER DIAMTER MEASUREMENTS}

The first step to measuring the fiber diameters was to set the appropriate scale for the images in Image J. To do this, the Edmund Optics Stage Micrometer image was opened in Image $\mathrm{J}$ (File $\rightarrow$ Open). In order to set the global scale, a line was drawn between a known distance and the pixel count was converted to the appropriate unit scale, using the pixel: length relationship. Since the stage micrometer is known to be $1 \mathrm{~mm} / 100$ divisions, a line was drawn across the window (8 divisions), shown in Figure 13 , and the divisions were counted and used in the following equation:

$1 \mathrm{~mm} / 100$ divisions $=\mathrm{X} \mathrm{mm} / 8$ divisions 


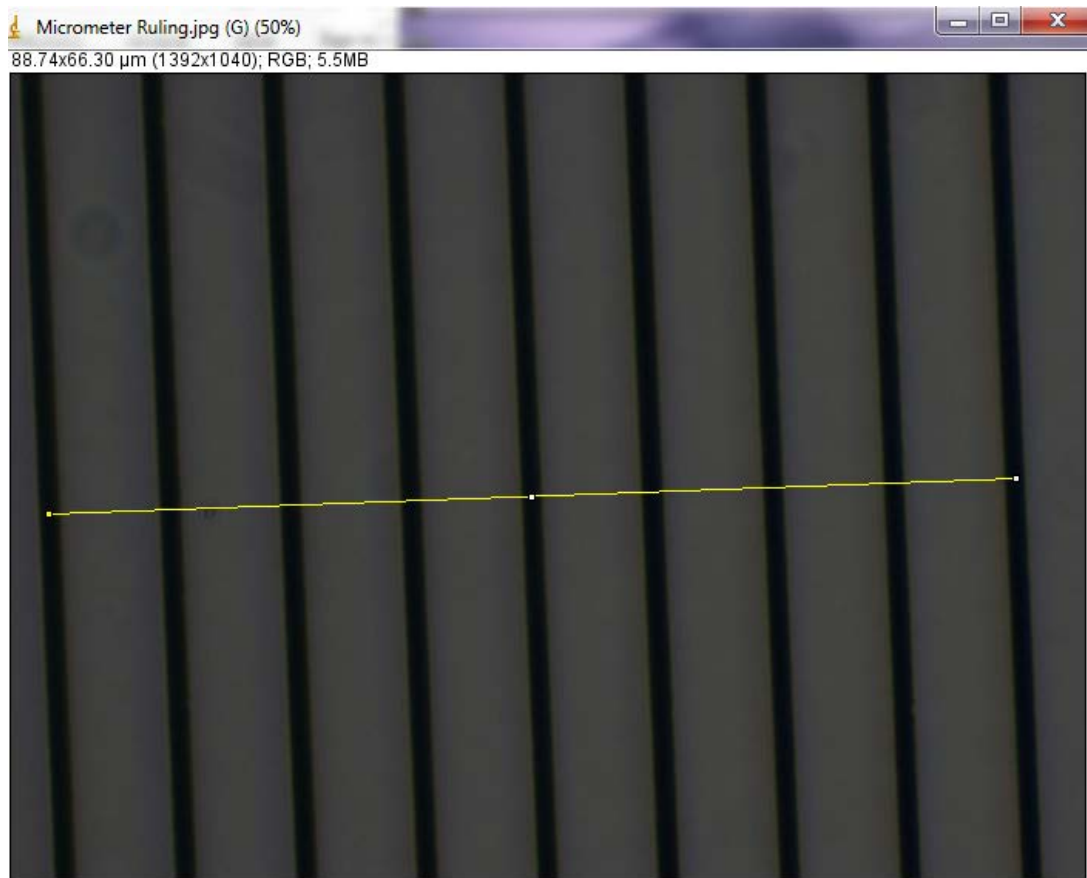

Figure 13: Example of setting a known distance in Image J (Line Selection tool)

The known distance between the eight divisions on the Image $\mathrm{J}$ window was $.08 \mathrm{~mm}$ or $80 \mu \mathrm{m}$. This value was entered into the known distance box using Analyze $\rightarrow$ Set Scale and the units were set to micrometers as shown in Figure 14.

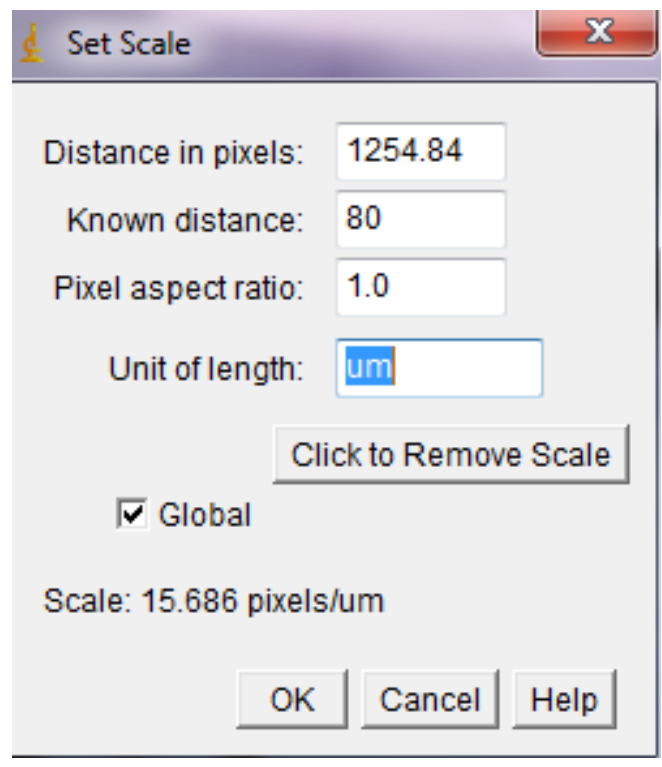

Figure 14: Example of setting the scale in Image J (Analyze $\rightarrow$ Set Scale) 
Each image to be analyzed was in a window of known distance $88.74 \times 66.30 \mu m$. Now that the scale was set for a known distance, it was necessary to measure each fiber diameter. This was done by clicking the Freehand Selection tool on the Image J toolbar and outlining the approximate circumference of each fiber shown in Figure 15.

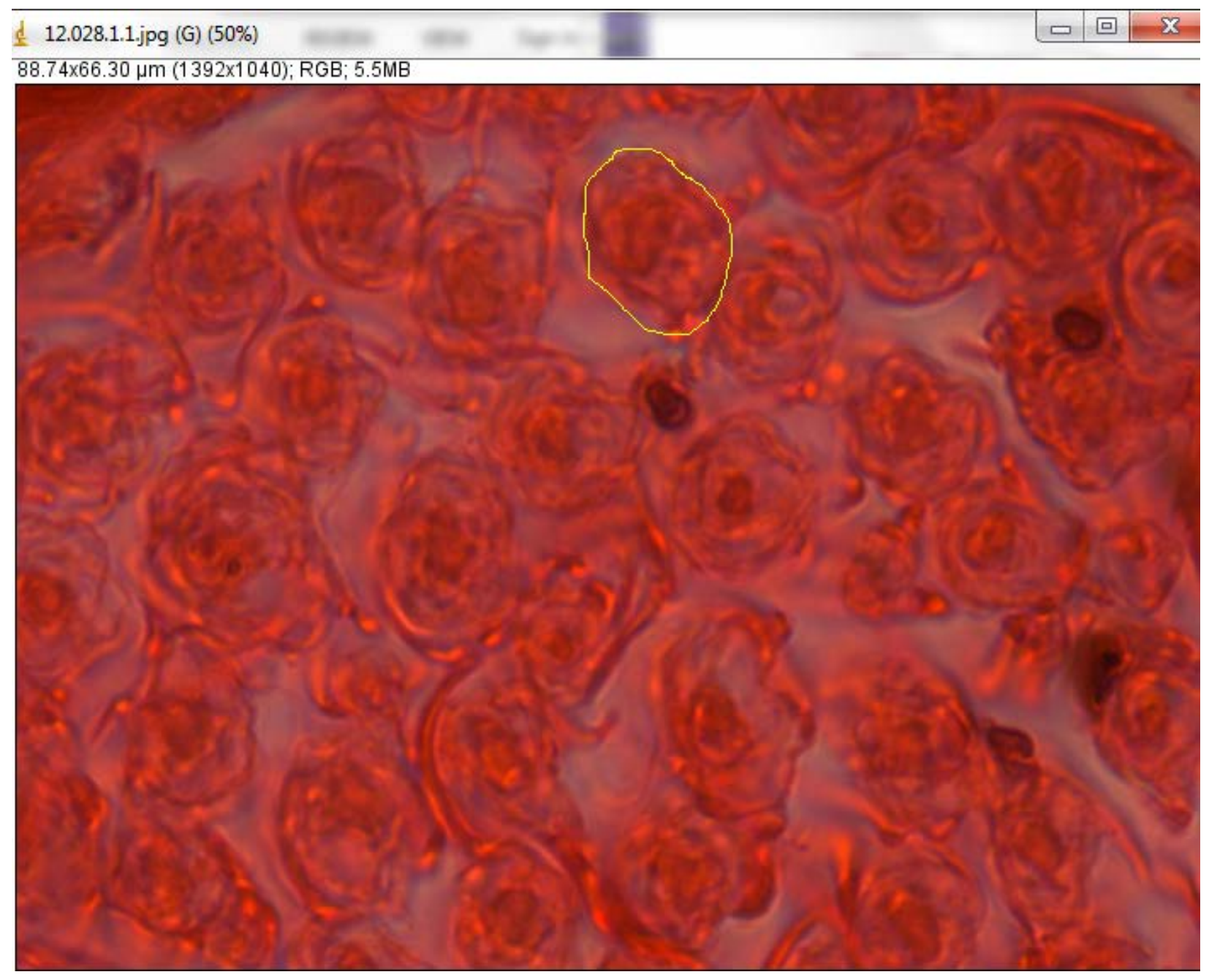

Figure 15: Example of outlining the approximate circumference of a nerve fiber (Freehand Selection tool)

To transfer the data from the selection to a data window, the function Analyze $\rightarrow$ Measure (or Ctrl M) was used and repeated for each individual fiber diameter. These data will provide the area, mean, max, and perimeter for the selection drawn by the Freehand Tool. These data were transferred to a Microsoft Excel file by saving the data window to a location on the 
computer. The area measurements and the perimeter measurements were then converted to a diameter measurement using the following equations for a circle:

Area $=\pi \mathrm{d}^{2} / 4 \quad$ or $\quad$ Circumference $=\pi \mathrm{d} \quad$ where $\mathrm{d}=$ diameter

A Microsoft Excel file was created with equations to do both of the aforementioned conversions, and an average of both conversions was used. An average was used because the fibers are not perfectly circular; therefore the diameter results using both the area equation and the circumference equation are not exact in value. Data was taken for each slide and saved onto Excel spreadsheets for use in the statistical analysis.

\section{$\underline{\text { STATISTICAL ANALYSIS }}$}

Histograms were created for each slide in order to provide a graphical representation of the distribution of the data for the analyzed images. Histograms are useful to show the probability of distribution of a given variable by depicting the frequencies of observations occurring in certain ranges of values. Statistical analysis was done in Microsoft Office Excel due to the availability of the Histogram tool of the Analysis ToolPak. To create a histogram, the data must be organized into two columns on the worksheet, one column for input data and the other for the bin numbers. Input data is the data that you want to analyze by using the Histogram tool. Bin numbers represent intervals. The histogram tool uses the bin spacing to sort the data into respective bins. The histogram tool in Excel counts the number of data points in each data bin. A data point is included in a particular data bin if the number is greater than the lowest bound and equal to or less than the largest bound for the data bin. The output of the histogram analysis is displayed on a new tab and shows a histogram table and a column chart that reflects the data in the histogram table. 
The next step was to ensure that there were two correct columns, an input variable column and a bin number column, to create the histogram. The input variable column was created from the diameter data that was calculated previously. To create the column of bin numbers, the following steps were followed:

1. The following function was used to create a count cell (Count) to get the total number of samples measured per slide:
a. =Count(A:A) [where $\mathrm{A}$ is the column]

2. The following equation was used to get the approximate number of recommended bins (Bins):

a. $=(\text { Count } * 2)^{\wedge}(1 / 3) \quad$ [where Count is the number from the previous step]

3. To get the interval to use between bins (interval), the following equation was used:

a. $=(\operatorname{MAX}(\mathrm{A}: \mathrm{A})-\mathrm{MIN}(\mathrm{A}: \mathrm{A})) /($ Bins $)$ [where Bins is answer from previous step]

4. Finally, the bin column was created by taking the minimum of the input column, adding the interval value (interval) previously calculated, and repeating for subsequent values, until the number of bins (bins rounded up) was reached.

Now that there was an input column and a bin column, the Data Analysis ToolPak for a histogram was used. The Data tab was selected, then Data Analysis. In the Data Analysis dialog box, Histogram was clicked, followed by OK. For the Input Range, the input variable column was selected, and for the Bin Range, the Bin column was selected. Under Output Options, New Worksheet Ply was selected and renamed, and Chart Output was selected followed by OK.

Figure 16 shows the output of the Histogram ToolPak. 


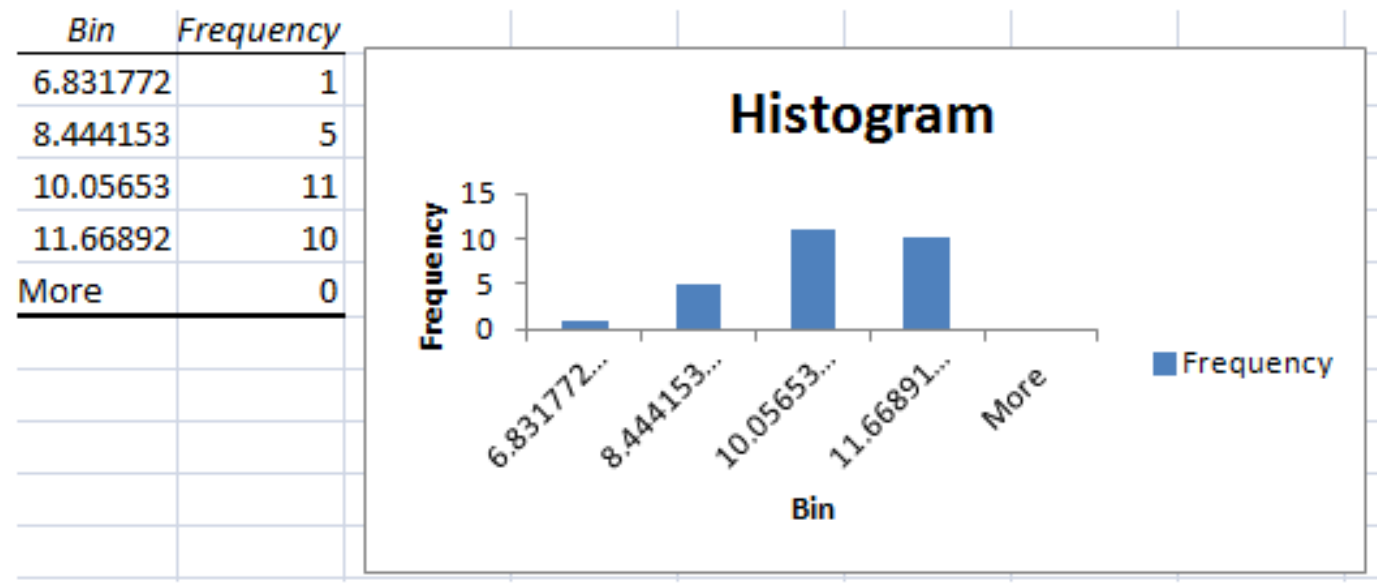

Figure 16: Original output using the Microsoft Office Excel ToolPak

Changes were then made to the original output in order to make the results more presentable. The changes that were made included: removing the More row, changing the title of the graph, changing colors of the bars on the graphs, making the bars on the graph touch to create the output shown in Figure 17 .

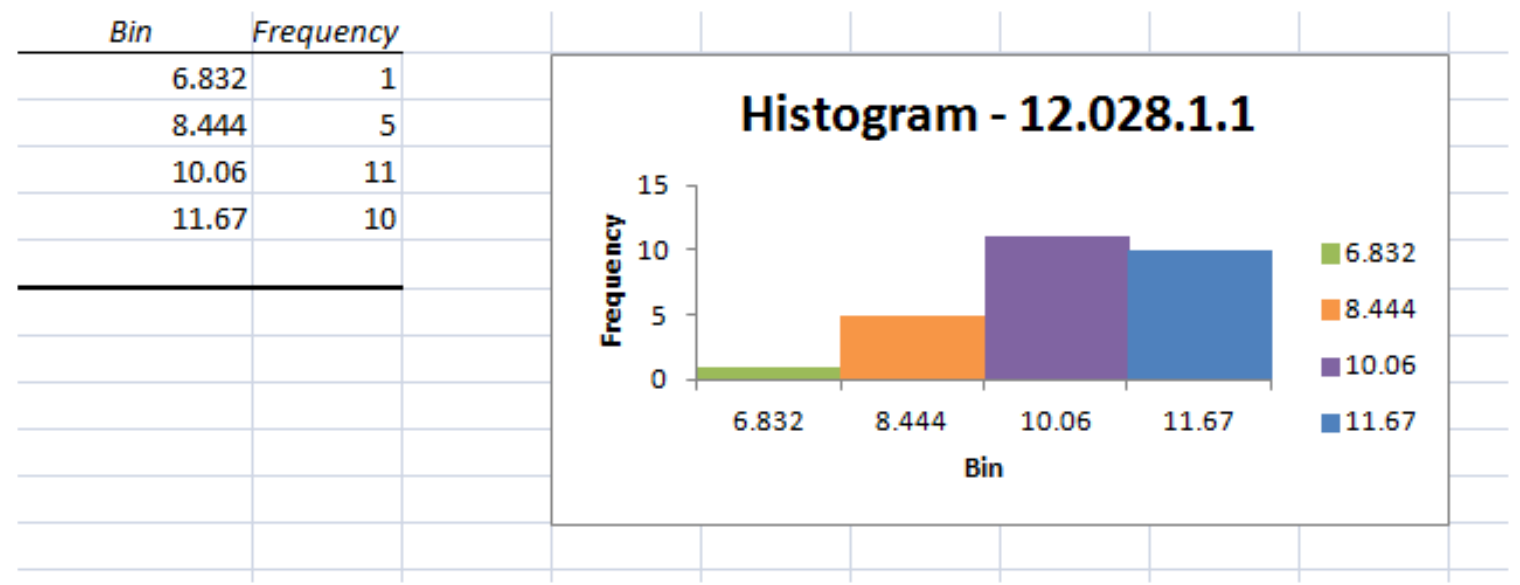

Figure 17: Altered output using Microsoft Office Excel ToolPak

The results from the histogram graphs were then analyzed to determine the probability of distribution for the diameters of the measured neurons. 


\section{CHAPTER IV: RESULTS}

After images were captured using Q Capture Pro 6.0, the nerve fiber diameters were counted and measured using Image $\mathrm{J}$ software. Actual images can be found in Appendix B. The results were transferred to Microsoft Office Excel where histograms were created for each slide observed. Raw data taken from images can be found in Appendix C. Histograms were created in order to provide a graphical representation of the distribution of the data for the analyzed images. Histograms are useful to show the probability of distribution of a given variable by depicting the frequencies of observations occurring in certain ranges of values. For all of the following histograms, bin sizes were created by using the procedure found in the Statistical Analysis section of Chapter III: Methods. The y-axis of the chart will represent the frequency, while the x-axis will show the diameters of the measured neurons separated into specified bins.

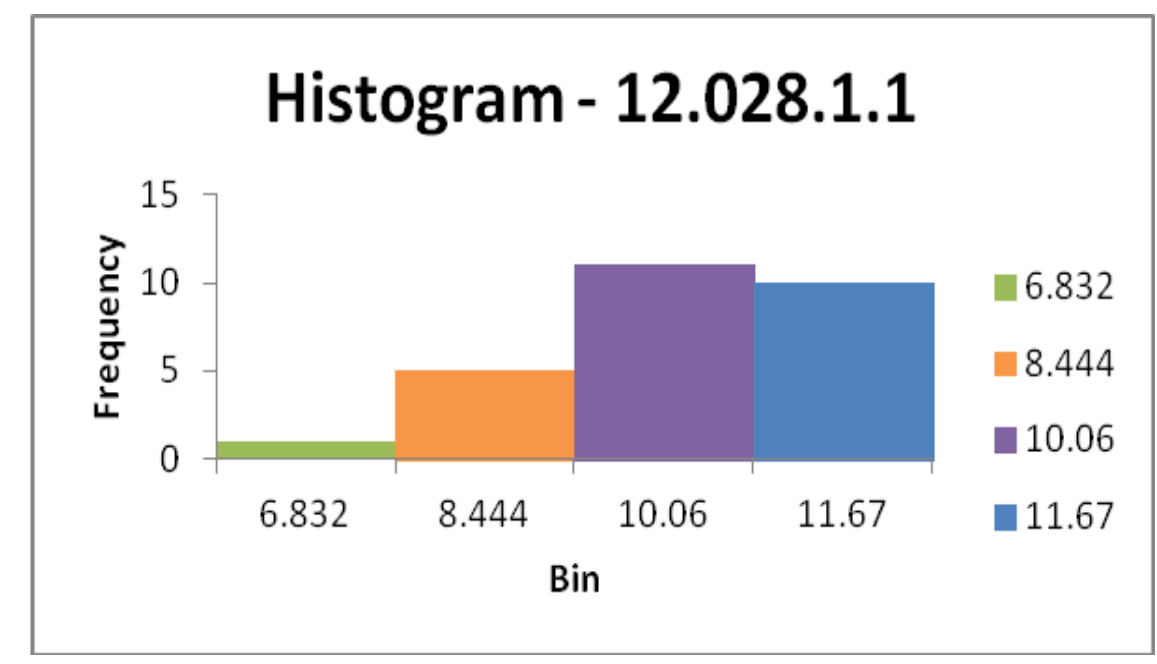

Figure 18: Histogram representing data from sample 12.028.1.1 


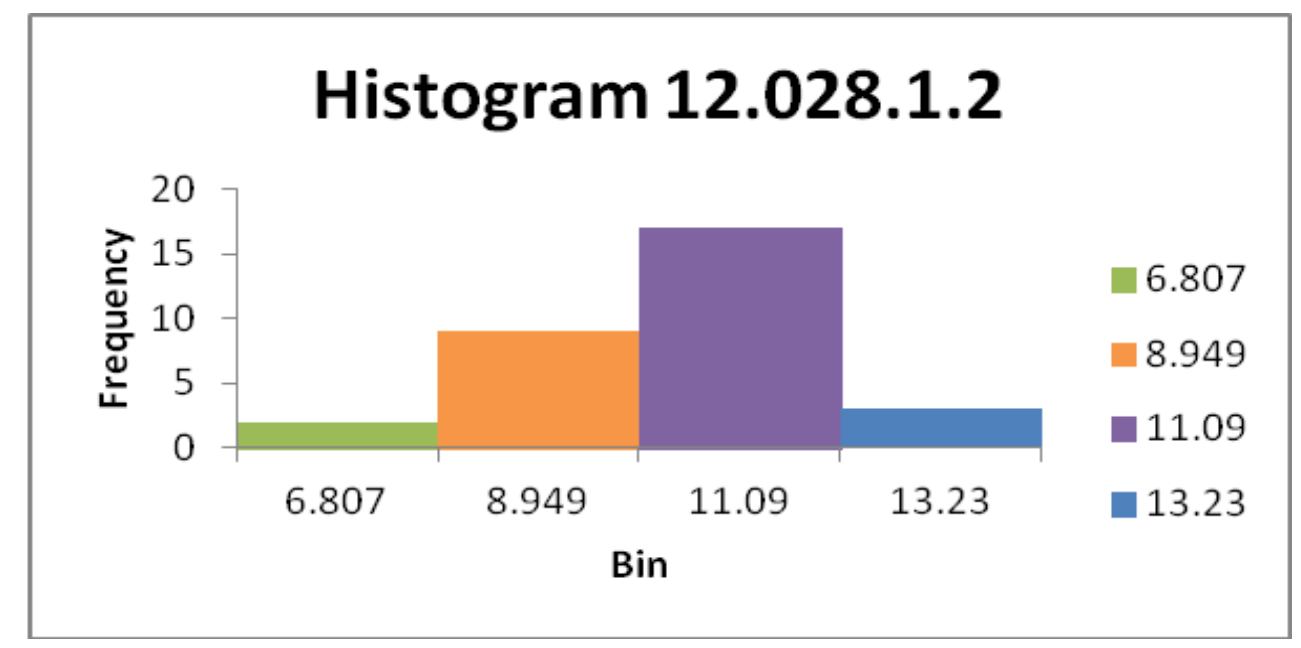

Figure 19: Histogram representing data from sample 12.028.1.2

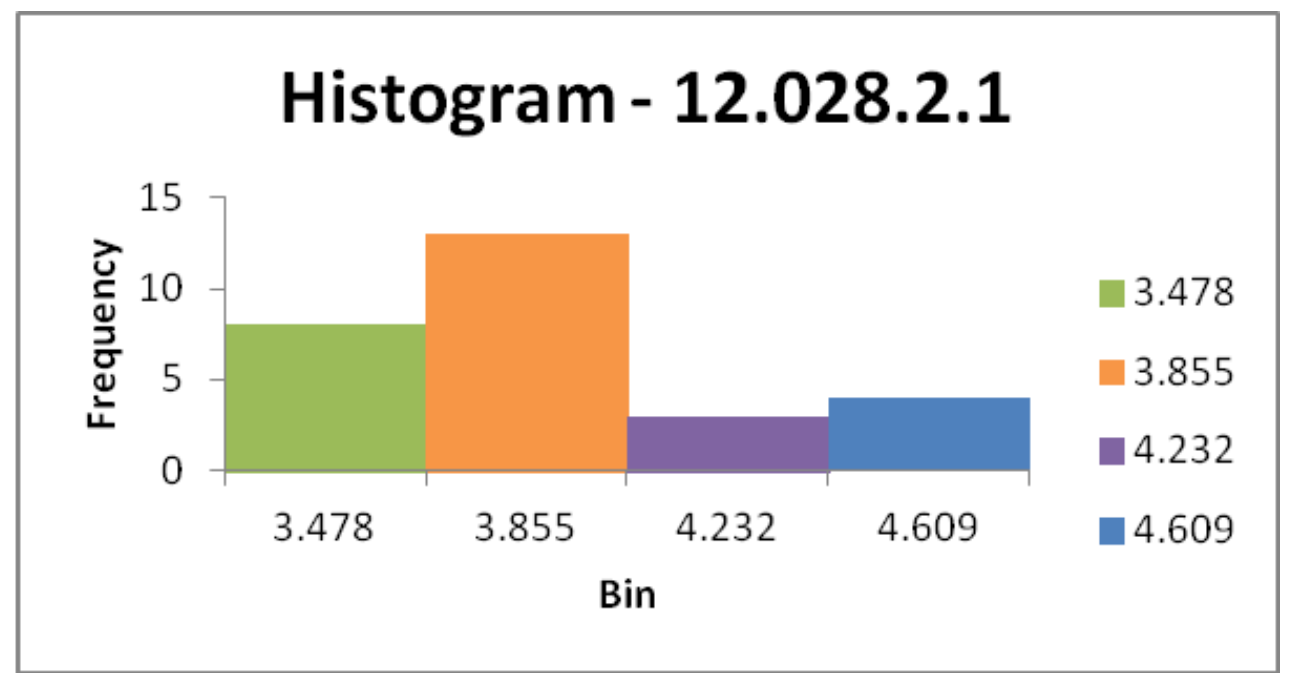

Figure 20: Histogram representing data from sample 12.028.2.1 


\section{Histogram - 12.028.2.2}

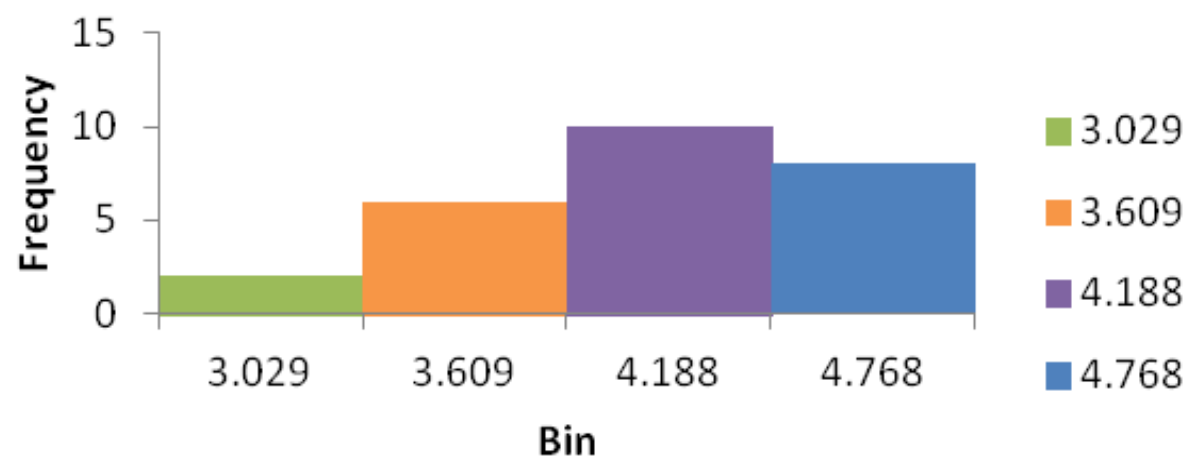

Figure 21: Histogram representing data from sample 12.028.2.2

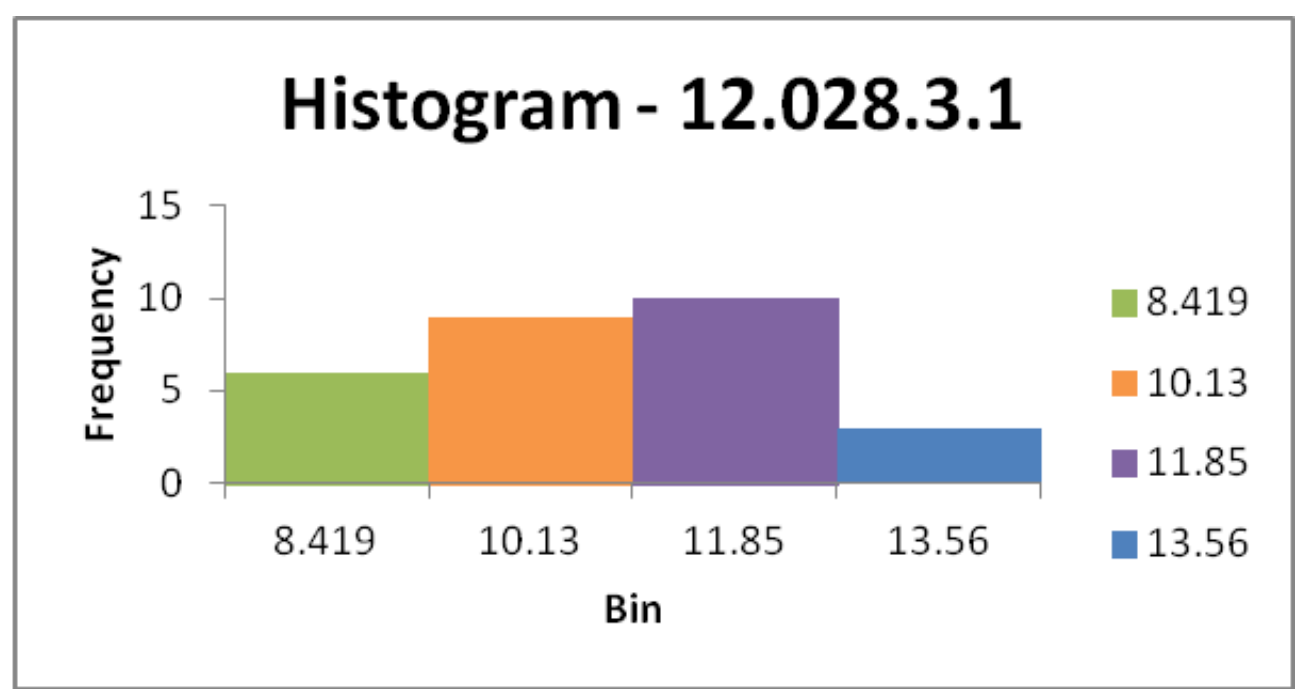

Figure 22: Histogram representing data from sample 12.028.3.1 


\section{Histogram - 12.028.3.2}

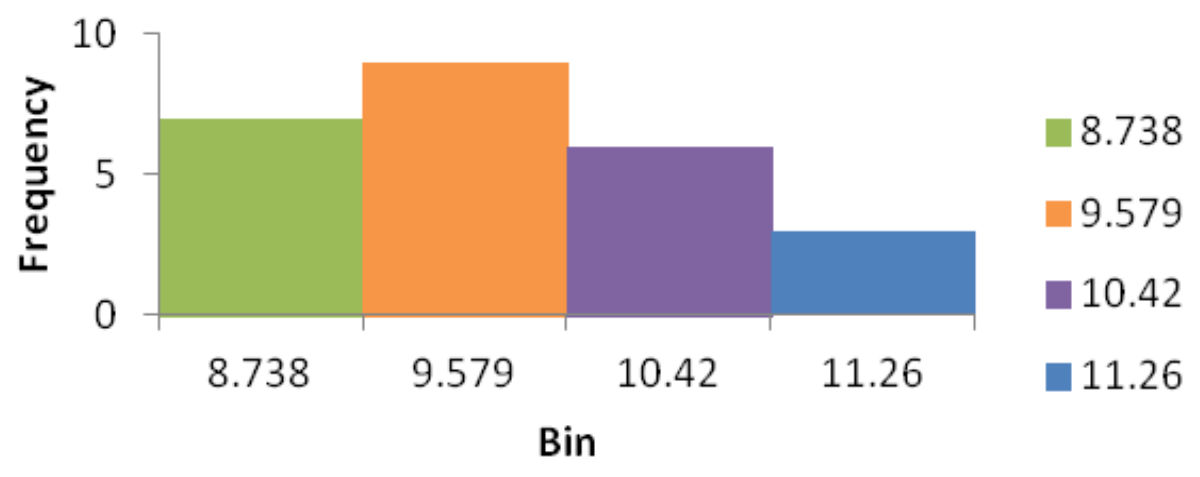

Figure 23: Histogram representing data from sample 12.028.3.2

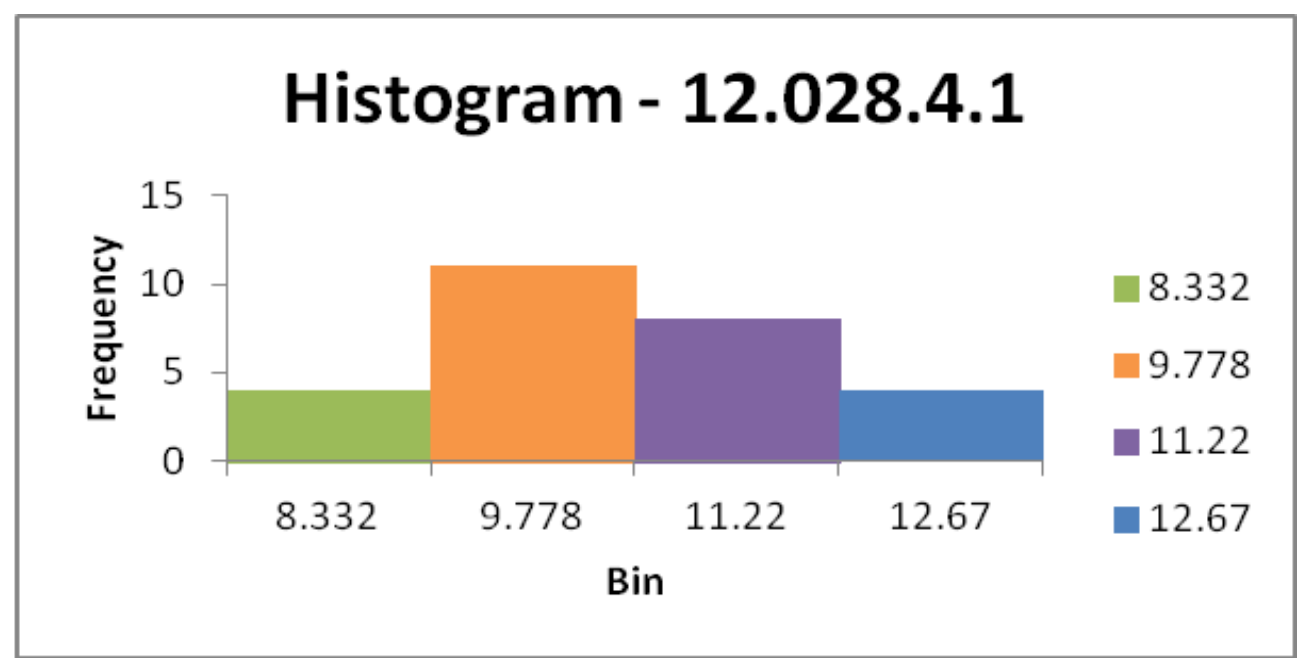

Figure 24: Histogram representing data from sample 12.028.4.1 


\section{Histogram - 12.028.5.1}

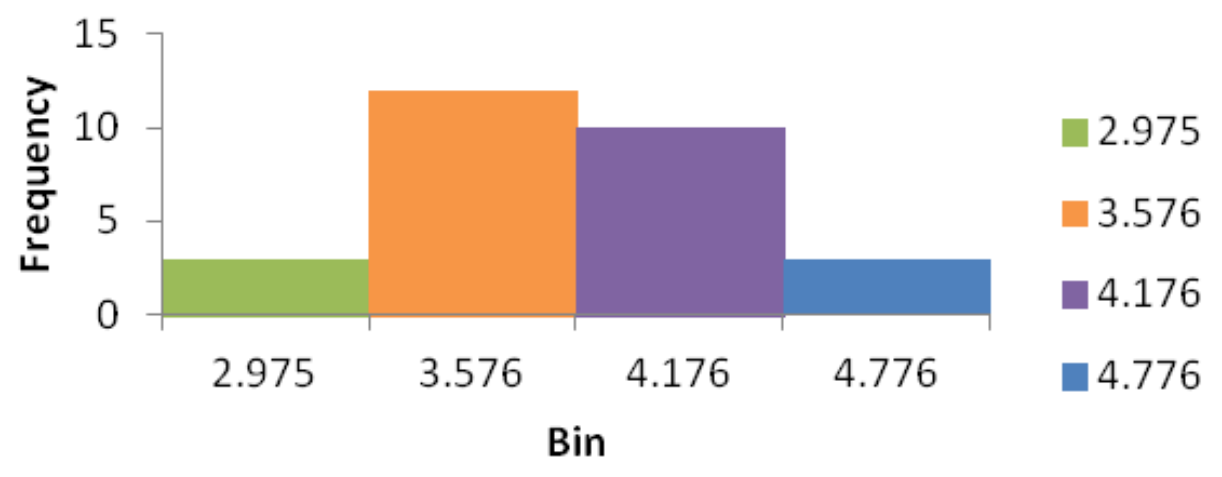

Figure 25: Histogram representing data from sample 12.028.5.1

\section{Histogram - 12.028.5.2}

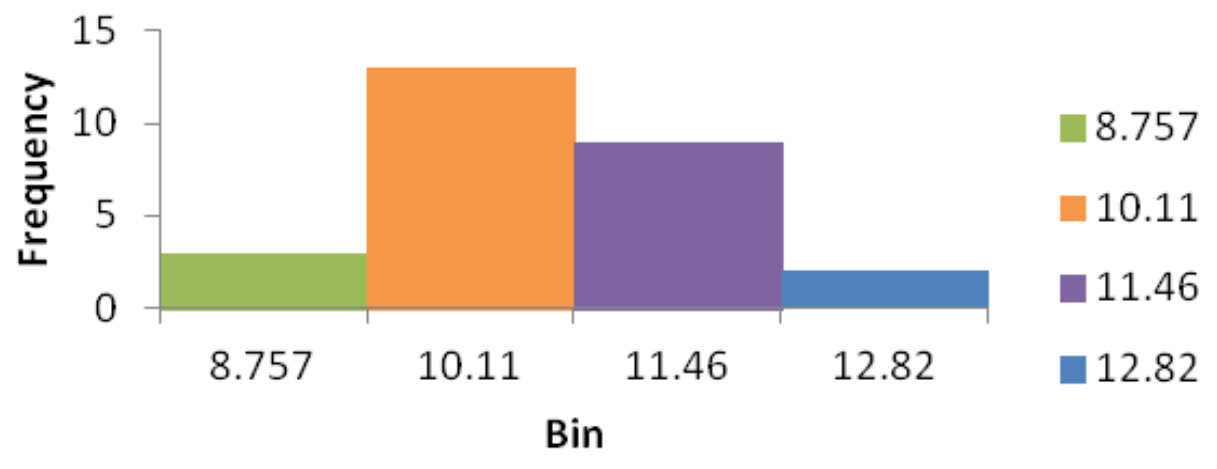

Figure 26: Histogram representing data from sample 12.028.5.2 


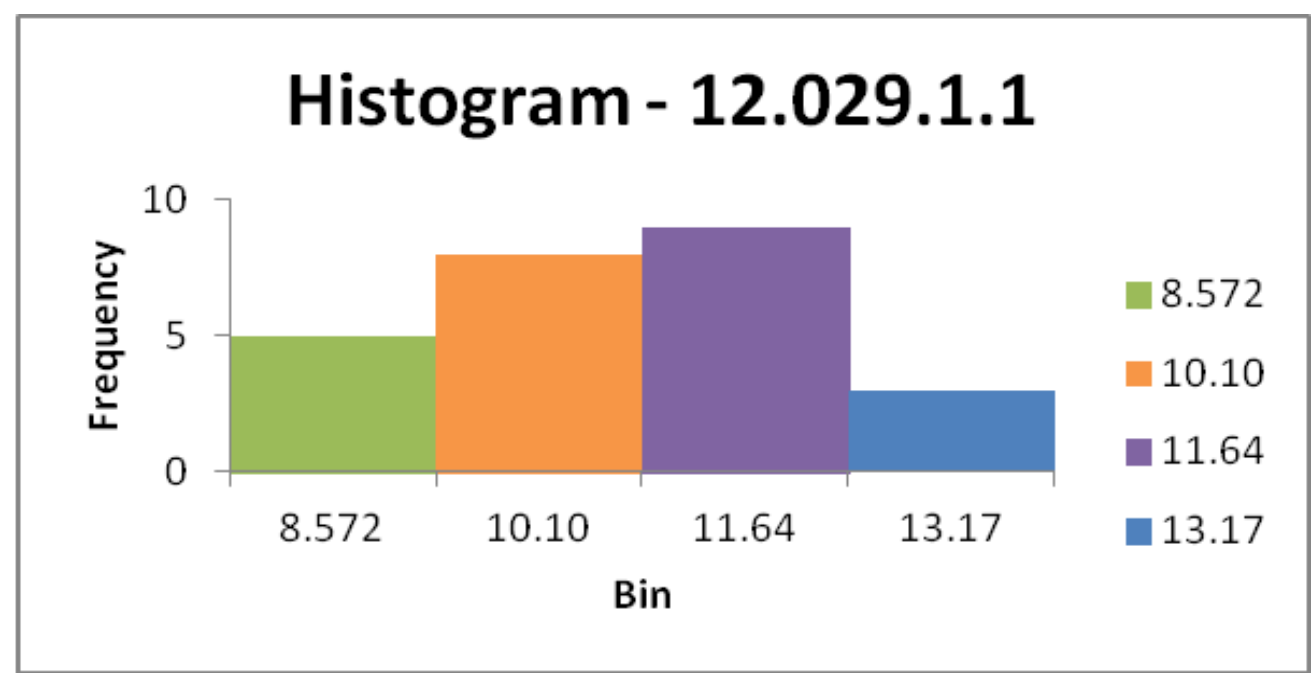

Figure 27: Histogram representing data from sample 12.029.1.1

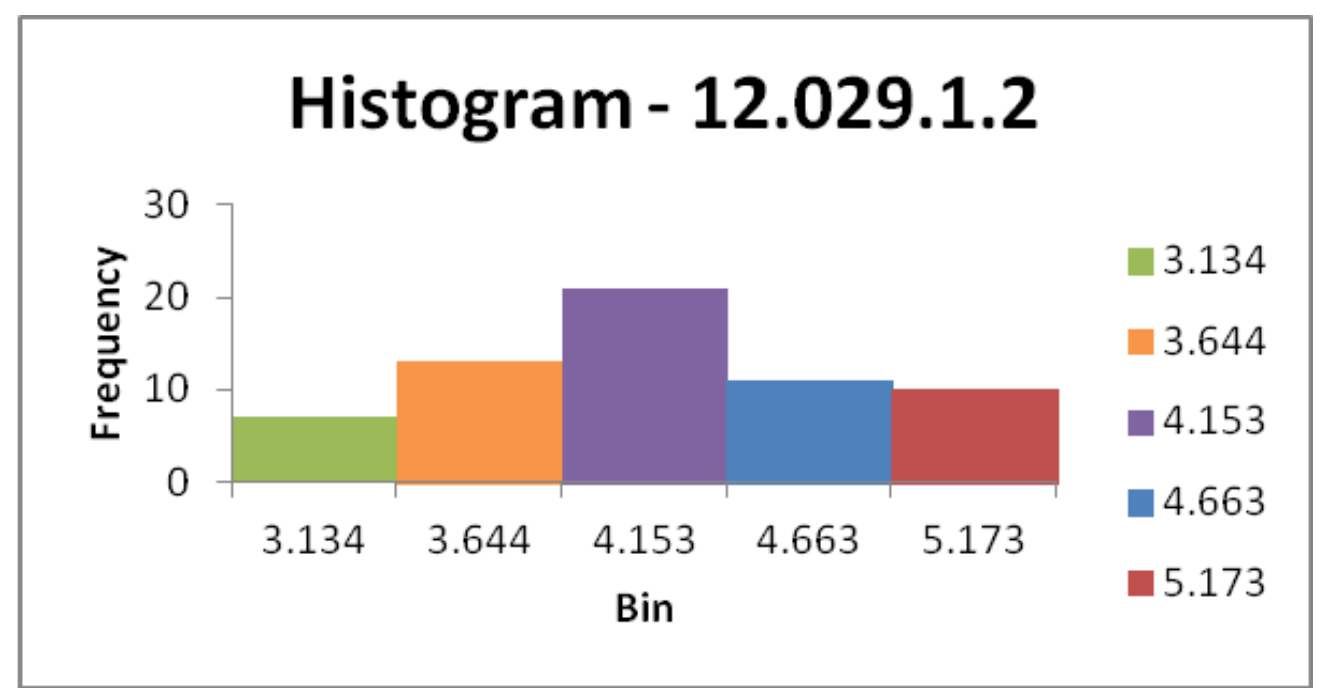

Figure 28: Histogram representing data from sample 12.029.1.2 


\section{Histogram - 12.029.2.1}

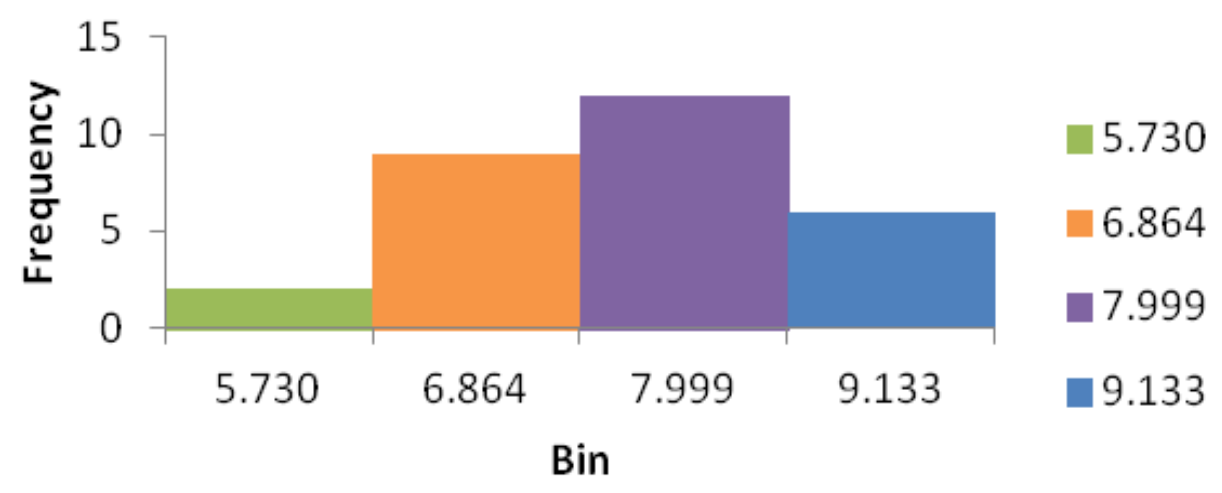

Figure 29: Histogram representing data from sample 12.029.2.1

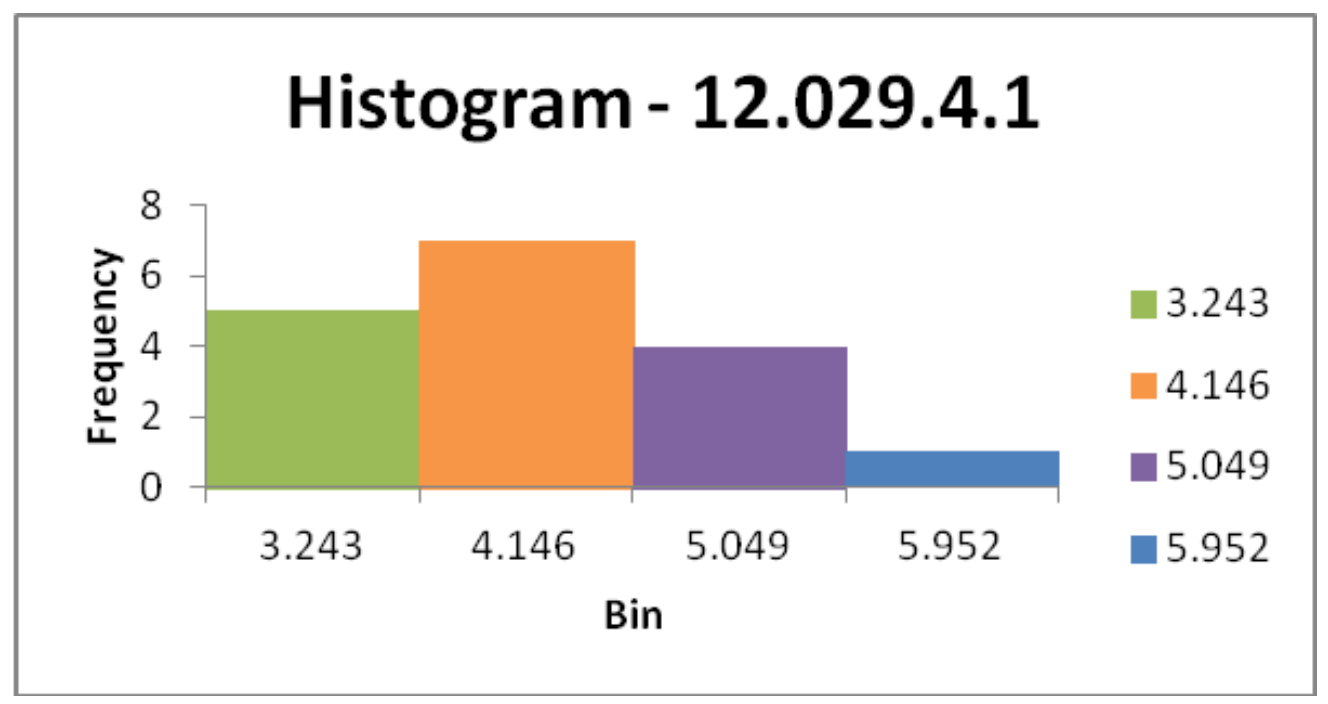

Figure 30: Histogram representing data from sample 12.029.4.1 


\section{Histogram - 12.029.5.1}

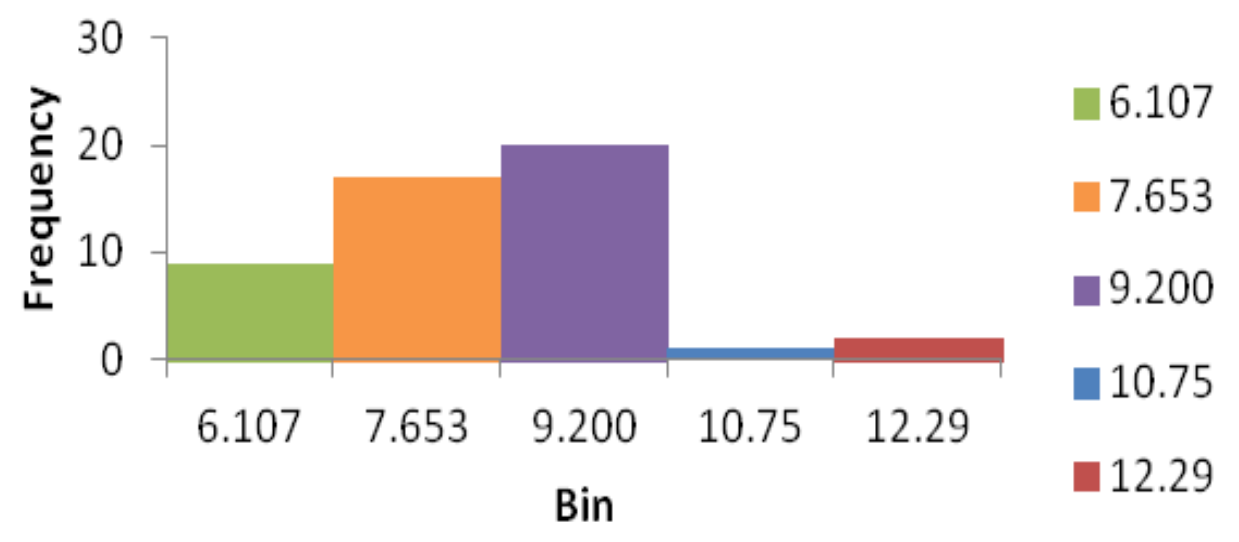

Figure 31: Histogram representing data from sample 12.029.5.1

Histogram - 12.029.5.2

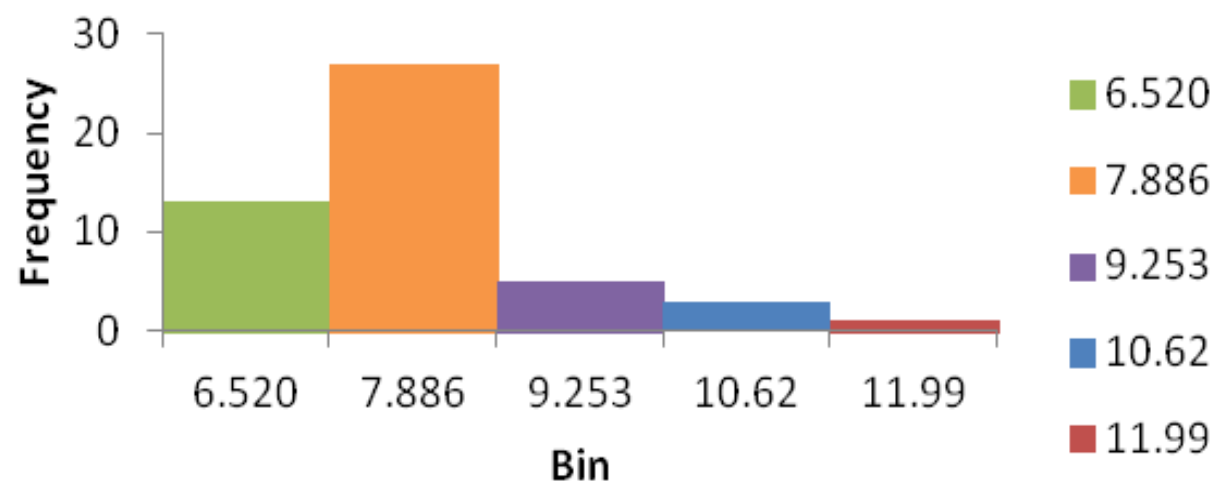

Figure 32: Histogram representing data from sample 12.029.5.2 


\section{Histogram 12.030.1.1}

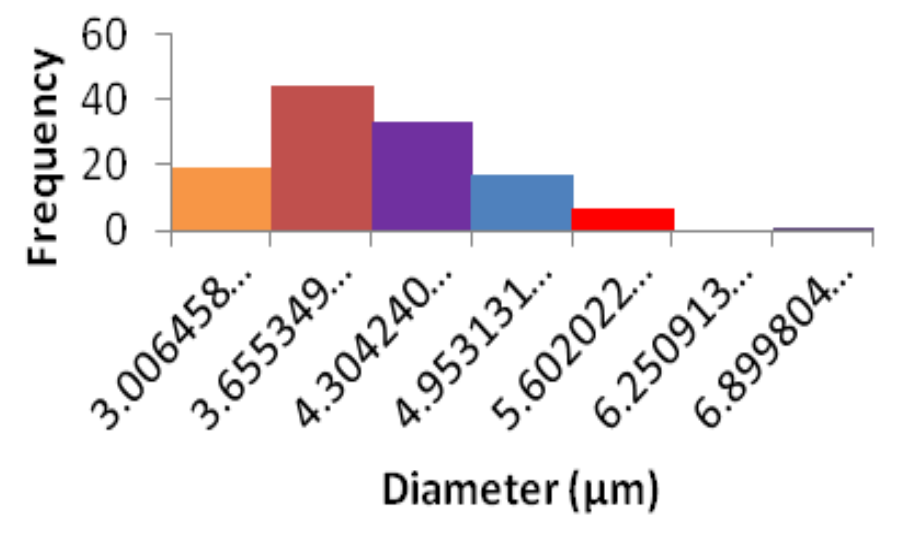

3.006458082

3.655349189

1.304240297

4.953131405

5.602022512

6.25091362

6.899804727

Figure 33: Histogram representing data from sample 12.030.1.1

\section{Histogram - 12.030.1.2}

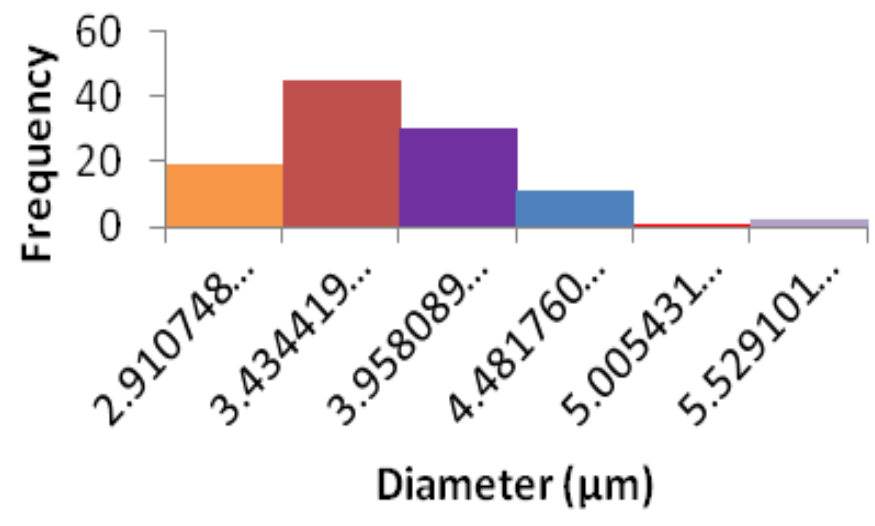

2.910748419

3.434419105

3.958089791

4.481760477

5.005431163

5.529101849

Figure 34: Histogram representing data from sample 12.030.1.2 


\section{Histogram - 12.030.2.1}

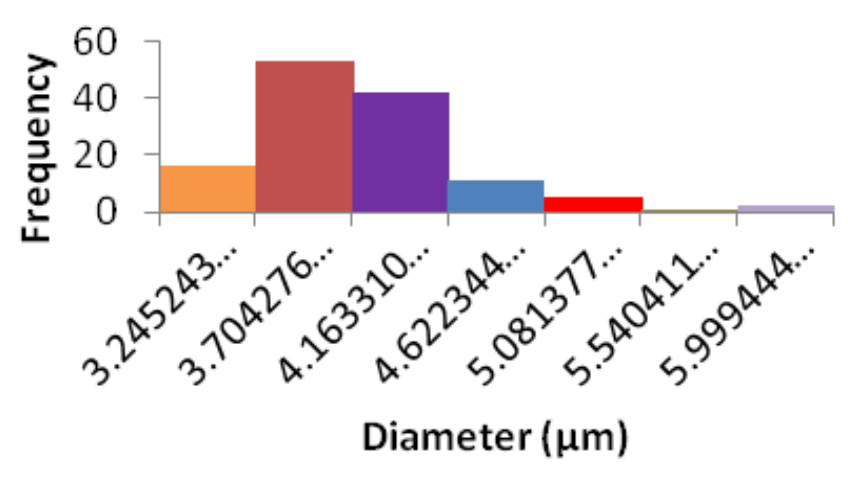

3.245243348

3.704276945

4.163310542

4.62234414

5.081377737

5.540411334

5.999444931

Figure 35: Histogram representing data from sample 12.030.2.1

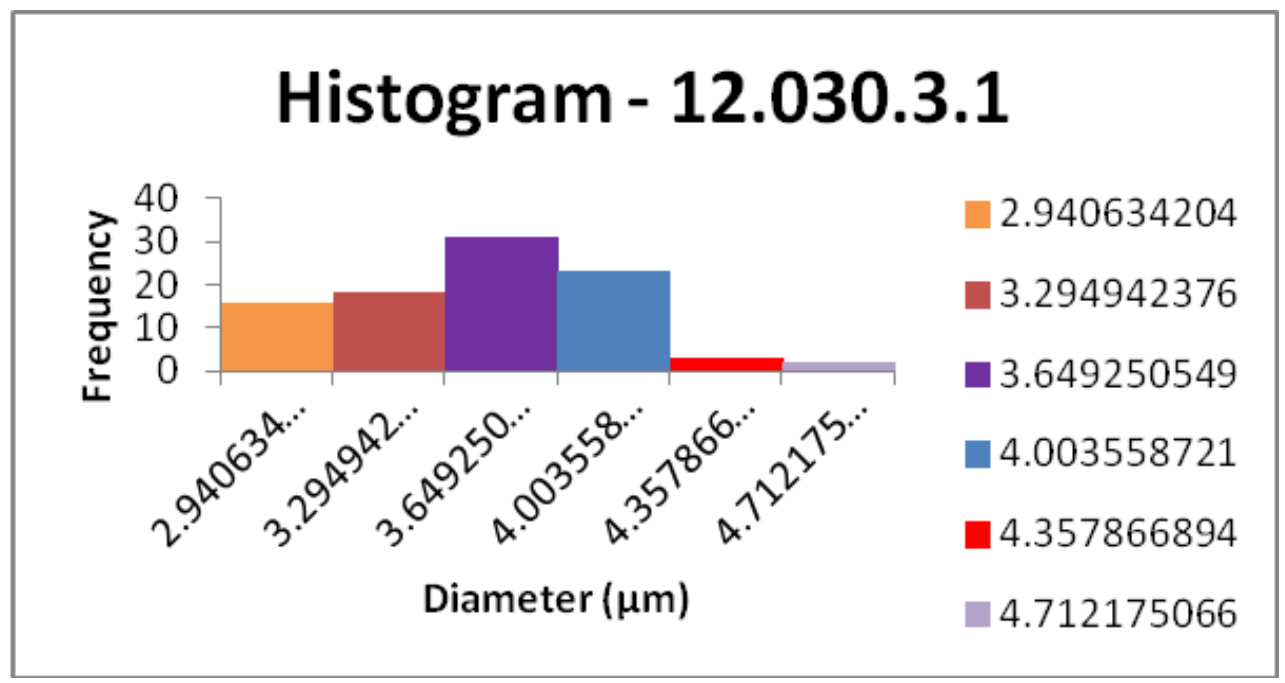

Figure 36: Histogram representing data from sample 12.030.3.1 


\section{Histogram - 12.030.3.2}

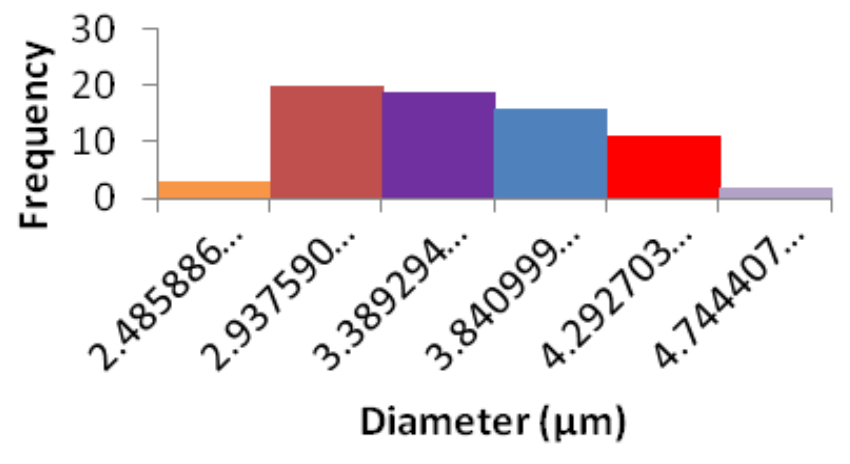

2.48588655

2.93759075

3.38929495

3.84099915

4.29270335

4.744407549

Figure 37: Histogram representing data from sample 12.030.3.2

\section{Histogram - 12.030.4.1}

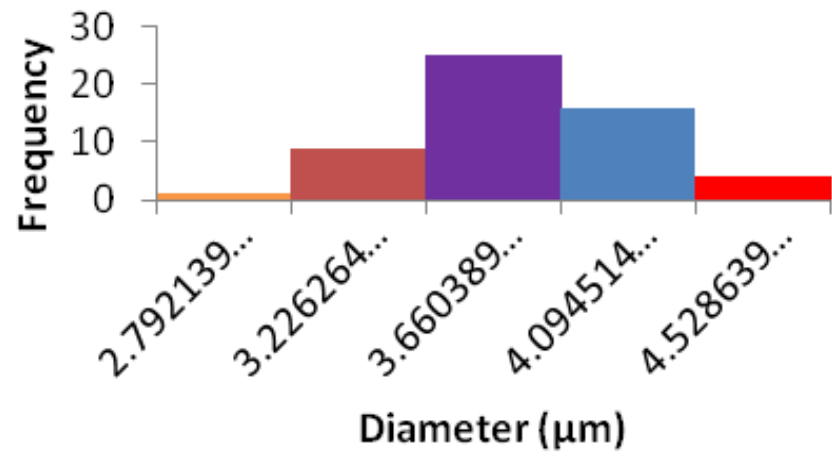

2.792139003

3.22626406

3.660389117

4.094514174

4.528639231

Figure 38: Histogram representing data from sample 12.030.4.1 


\section{Histogram - 12.030.5.1}

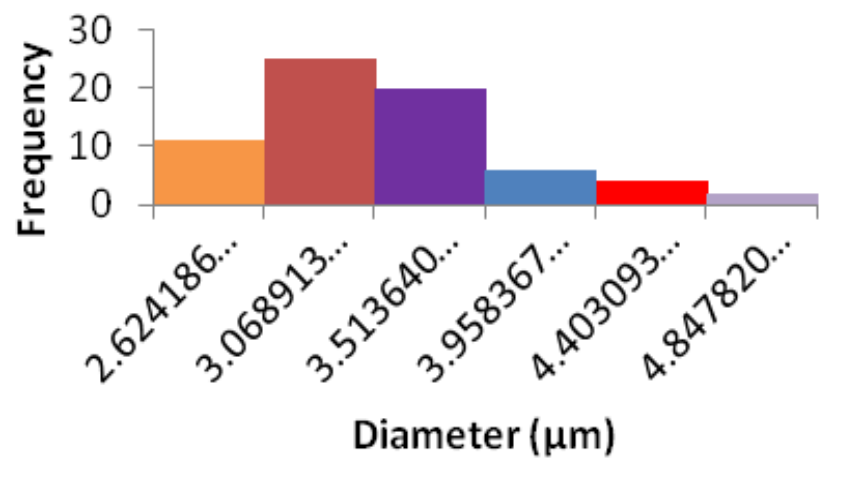

2.624186735

3.068913493

3.513640252

3.95836701

4.403093769

4.847820527

Figure 39: Histogram representing data from sample 12.030.5.1

The following histograms were created by using the information from each individual sample and combining that data to create a histogram from each tissue block. These histograms show the average diameter for every fiber measured from each sample block. These results can help differentiate whether different sample blocks had varying fiber diameters.

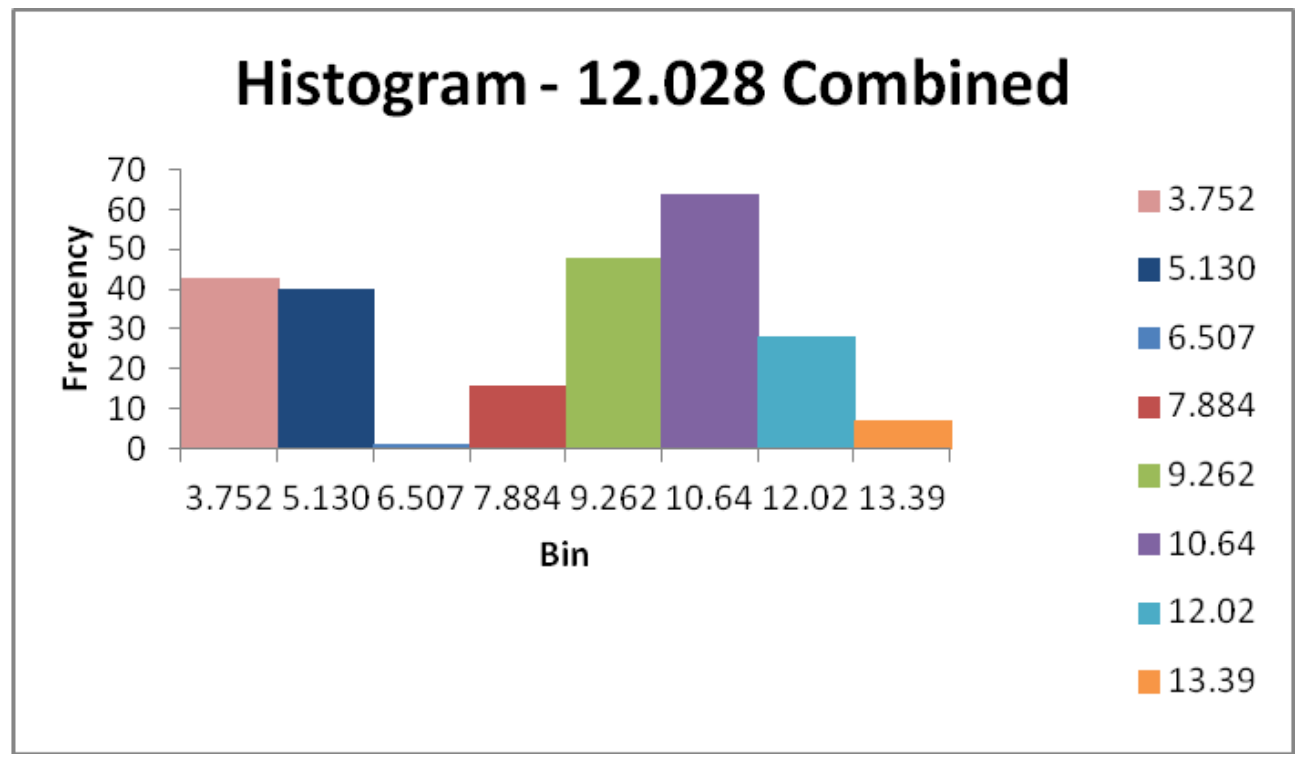

Figure 40: Histogram representing combined data taken from all samples from tissue block 12.028 


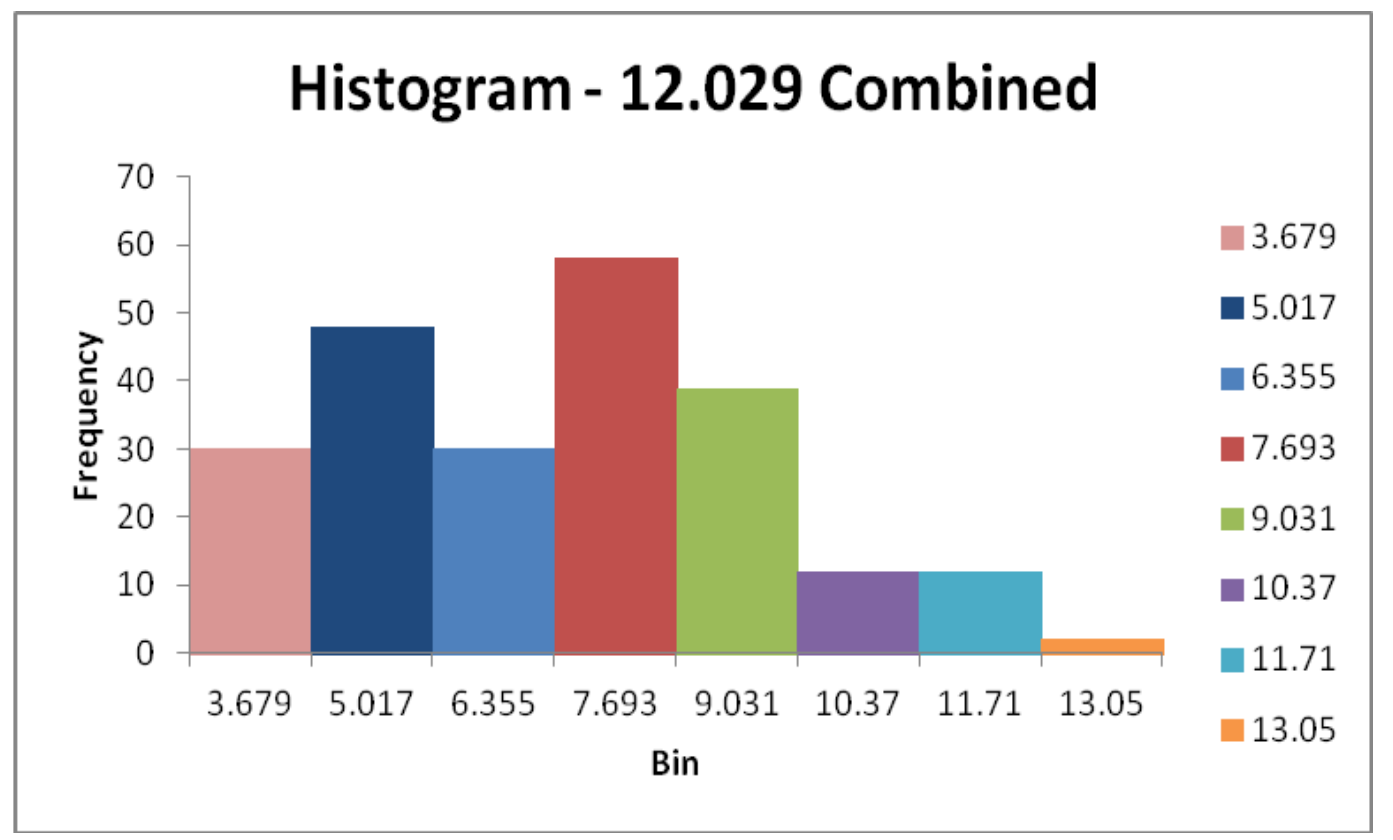

Figure 41: Histogram representing combined data taken from all samples from tissue block 12.029

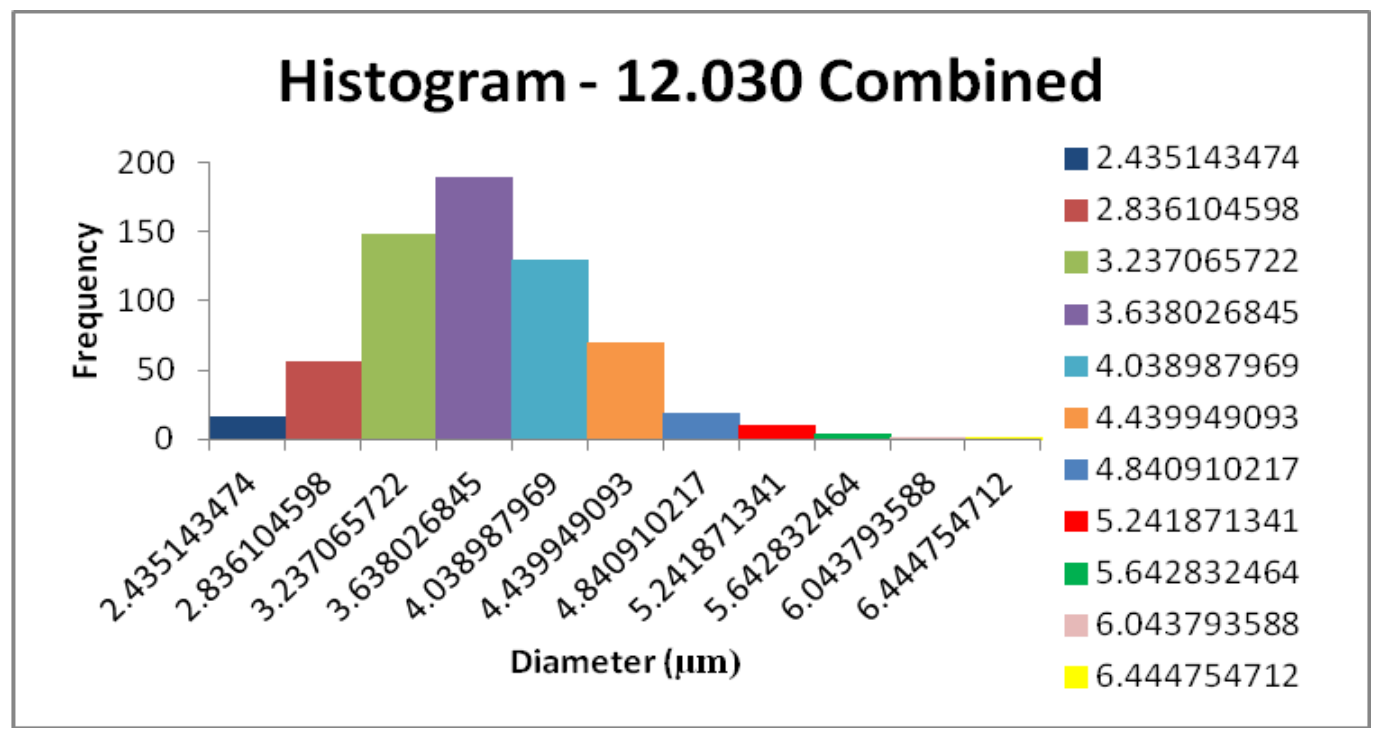

Figure 42: Histogram representing combined data taken from all samples from tissue block 12.030 


\section{CHAPTER V: DISCUSSION}

The primary goal for this experiment was to acquire the results necessary to prove the efficacy of the theory previously presented by Szlavik et al. [3] for characterization of peripheral nerve fiber size distributions by group delay. The experiment presented a technique based on decomposition of the maximal compound evoked potential and subsequent determination of the group delay of the contributing nerve fibers. The fiber group delay was then utilized as an initial estimation of the nerve fiber size distribution and the associated temporal propagation delays of the single-fiber-evoked potentials to a reference electrode. The results of the simulation study demonstrated that the technique presented could, with reasonable accuracy, retrieve the fiber size distribution in the presence of recording noise for a wide range of SNR values.

In the present study, three rat sciatic nerves were extracted, embedded in paraffin wax, sectioned, stained, imaged with brightfield microscopy, and analyzed for results. One of the rat nerves served as the control nerve (12.028), while the other two nerves belonged to the experimental model chemically induced with diabetes (12.029 and 12.030). The findings from this experiment show the frequency of nerve fiber diameters for each slide analyzed using brightfield microscopy. Also, the results from each individual slide were combined to show the frequency of nerve fiber diameters per paraffin wax block.

For the paraffin wax block labeled 12.028 (control), there was a wide range of nerve fiber diameter values occurring between approximately $3 \mu \mathrm{m}$ and $14 \mu \mathrm{m}$. The highest frequency (64) occurred between $9.262 \mu \mathrm{m}$ and $10.64 \mu \mathrm{m}$. Although the second highest frequency occurred between 7.884 and $9.262 \mu \mathrm{m}$, there was also a high frequency of values occurring under 5.130 $\mu \mathrm{m}$.

For the paraffin wax block labeled 12.029 (experimental), there was also a wide range of nerve fiber diameter values occurring between approximately $3 \mu \mathrm{m}$ and $14 \mu \mathrm{m}$. The highest 
frequency (58) occurred between 6.355-7.693 $\mu \mathrm{m}$. The remaining diameters were more focused between approximately 3.5 - $9 \mu$ m; relatively few diameters were higher than $9.031 \mu \mathrm{m}$.

For the paraffin wax block labeled 12.030 (experimental), there was a more expected range of results shown in the combined histogram. The most frequent diameters (190) occurred between the range of 3.237 and $3.638 \mu \mathrm{m}$, with a bell-shaped curve expanding outwards, with few diameters occurring after $4.440 \mu \mathrm{m}$.

Blocks 12.028 and 12.029 showed no trend in results, varying between smaller diameter fibers and larger diameter fibers, while block 12.030 showed a trend where most of the fiber diameter were under $4.440 \mu \mathrm{m}$. The control block did in fact show a wider range of values ( $3 \mu \mathrm{m}-$ $14 \mu \mathrm{m})$, while the experimental blocks generally showed a smaller diameter $(<9 \mu \mathrm{m})$.

The nerve fiber diameters measured for all of the combined samples ranged from approximately $2.5 \mu \mathrm{m}-14 \mu \mathrm{m}$. Boyd and Davey [39] published Composition of Peripheral Nerves which shows results that peripheral nerve fiber diameters can range from a few microns to twenty microns. The measured diameters found in this research are within range of previous publications, and are therefore realistic and can be used in future studies.

Some limitations were found during the execution of this experiment that may have led to incorrect, inconclusive, or unusable results. One of the main limitations was in the process of staining. The hematoxylin and eosin stains had been about two years expired when they were used which could have adverse effects on the staining results. Also, as mentioned before, some of the staining baths were reused, or not changed immediately before use, which could have also negatively affected the results of the staining process. There was a dramatic improvement in results when the solutions were changed prior to staining versus when solutions were reused from previous experiments. In the future, new and fresh hematoxylin, eosin, bluing solution, clarifier, xylene, and ethanol should be used to achieve the best looking images. 
Some possible work for the future to improve results would be to change the method of embedding and staining. The gold standard protocol for processing peripheral nerve tissue to obtain high resolution imaging of myelinated fibers is resin embedding after $2 \%$ osmium tetroxide postfixation [17]. The advantages of staining with osmium tetroxide before embedding are: (1) it prevents myelin sheath swelling, which usually occurs during paraffin embedding, thanks to its action as a lipid fixative; (2) it provides the typical dark and sharp myelin stain, which greatly facilitates the identification of nerve fibers. Measuring the myelin sheath would help discriminate between myelinated and unmyelinated fibers, which would have been helpful when determining which type of peripheral nerve fibers are being affected. Another method to detect the myelin sheaths more clearly would be to use Masson's trichrome counterstaining, which in particular permits clear imaging of the nerve's connective structures [17].

In conclusion, the goal of this study was to create data to be used in an algorithm to prove the efficacy of a novel method of characterizing peripheral nerve fiber distributions by group delay. The results found during execution of this experiment will be used to support continuing research for a novel method to characterize peripheral neuropathies using group delay. 


\section{BIBLIOGRAPHY}

1. Tavee, J., and L. Zhou. "Small Fiber Neuropathy: A Burning Problem." Cleveland Clinic Journal of Medicine 76.5 (2009): 297-305. Web.

2. Brannagan, Thomas H., III. "Current Issues in Peripheral Neuropathy." Journal of the Peripheral Nervous System 17 (2012): 1-3. Web.

3. Szlavik, R.b. "A Novel Method for Characterization of Peripheral Nerve Fiber Size Distributions by Group Delay." IEEE Transactions on Biomedical Engineering 55.12 (2008): 2836-840. Web.

4. Harati, Yadollah. "Diabetic Peripheral Neuropathies." Annals of Internal Medicine 107.4 (1987): 546. Web.

5. Dorfman, L. J., K. L. Cummins, G. M. Reaven, J. Ceranski, M. S. Greenfield, and L. Doberne. "Studies of Diabetic Polyneuropathy Using Conduction Velocity Distribution (DCV) Analysis." Neurology 33.6 (1983): 773. Web.

6. Shier, David, Jackie Butler, and Ricki Lewis. Hole's Human Anatomy \& Physiology. 11th ed. Boston, MA: McGraw-Hill Higher Education, 2007. Print.

7. Tortora, Gerard J., and Bryan Derrickson. Principles of Anatomy and Physiology. 12th ed. Hoboken, NJ: John Wiley \& Sons, 2010. Print.

8. Engstrom, John W. "Clinical Autonomic Disorders: Evaluation and Management. Edited by Phillip A. Low, Boston, Little, Brown, 1993, 832 Pp, Illustrated, \$165.00." Annals of Neurology 35.2 (1994): 250. Web.

9. Hoitsma, E., J.p.h. Reulen, M. De Baets, M. Drent, F. Spaans, and C.g. Faber. "Small Fiber Neuropathy: A Common and Important Clinical Disorder." Journal of the Neurological Sciences 227.1 (2004): 119-30. Web.

10. Köller, Hubertus, Bernd C. Kieseier, Sebastian Jander, and Hans-Peter Hartung. "Chronic Inflammatory Demyelinating Polyneuropathy." New England Journal of Medicine 352.13 (2005): 1343-356. Web.

11. Zhang, Chao, Bingdi Xie, Xiaown Li, and Yuanrong Yao. "Contact Heat-evoked Potentials as a Useful Means in Patients with Guillain-Barre Syndrome." Neurol Sci (2014). Web.

12. Soto, Nathan D. Characterizing Nerve Fiber Activation by Varying Fiber Diameter and Depth within a Conductive Medium: A Finite Element Approach. Thesis. California Polytechnic State University, San Luis Obispo, CA, 2011. Print.

13. Parker, P. A., and P. Kelly. "Nerve Conduction Velocity Measurement Techniques." Journal of Clinical Engineering 7.2 (1982): 153-58. Web. 
14. Mccomas, A. J., P. R. W. Fawcett, M. J. Campbell, and R. E. P. Sica. "Electrophysiological Estimation of the Number of Motor Units within a Human Muscle." Journal of Neurology, Neurosurgery \& Psychiatry 34.2 (1971): 121-31. Web.

15. Nicolas, Sammy Paul., and Robert Bruce Szlavik. Implementation of Novel Group Delay Decomposition Method and Surgical Protocol for Assessing Peripheral Neuropathy. Thesis. California Polytechnic State University, San Luis Obispo, CA, 2012. Print.

16. Marieb, Elaine N., and Katja Hoehn. Anatomy \& Physiology. Third ed. San Francisco, CA: Pearson/Benjamin Cummings, 2008. Print.

17. Scipio, Federica Di, Stefania Raimondo, Pierluigi Tos, and Stefano Geuna. "A Simple Protocol for Paraffin-embedded Myelin Sheath Staining with Osmium Tetroxide for Light Microscope Observation." Microscopy Research and Technique 71.7 (2008): 497502. Web.

18. Winer, J. B. "An Update in Guillain-Barré Syndrome." Autoimmune Diseases 2014 (2014): 1-6. Web.

19. Freeman, Roy. "Autonomic Peripheral Neuropathy." Neurologic Clinics 25.1 (2007): 277-301. Web.

20. Kerasnoudis, Antonios, Kalliopi Pitarokoili, Volker Behrendt, Ralf Gold, and Min-Suk Yoon. "Correlation of Nerve Ultrasound, Electrophysiological, and Clinical Findings in Post Guillain-Barré Syndrome." Journal of the Peripheral Nervous System 18.3 (2013): 232-40. Web.

21. Gonzalez-Cueto, J.a., and P.a. Parker. "Deconvolution Estimation of Nerve Conduction Velocity Distribution." IEEE Transactions on Biomedical Engineering 49.2 (2002): 14051. Web.

22. Franssen, Hessel, and Dirk C.g. Straver. "Pathophysiology of Immune-mediated Demyelinating Neuropathies-part I: Neuroscience." Muscle \& Nerve 48.6 (2013): 851-64. Web.

23. Franssen, Hessel, and Dirk C.g. Straver. "Pathophysiology of Immune-mediated Demyelinating Neuropathies-Part II: Neurology." Muscle \& Nerve 49.1 (2014): 4-20. Web.

24. Tesfaye, S., A. J. M. Boulton, P. J. Dyck, R. Freeman, M. Horowitz, P. Kempler, G. Lauria, R. A. Malik, V. Spallone, A. Vinik, L. Bernardi, and P. Valensi. "Diabetic Neuropathies: Update on Definitions, Diagnostic Criteria, Estimation of Severity, and Treatments." Diabetes Care 33.10 (2010): 2285-293. Web.

25. Vigeh, Arya. "Investigation of a Simulated Annealing Cooling Schedule Used to Optimize the Estimation of the Fiber Diameter Distribution in a Peripheral Nerve Trunk." Thesis. California Polytechnic State University, San Luis Obispo, CA, 2011. Web. 
26. Chen, Gin-Shin, and Yee-Fun Lee. "Measurement of Ultrasonic Attenuation in Diabetic Neuropathic Sciatic Nerves for Diagnostic and Therapeutic Applications." Web.

27. Chen, Gin-Shin, Yee-Fun Lee, and Jung-Sung Cheng. "Measurement of Ultrasonic Attenuation in Diabetic Neuropathic Sciatic Nerves for Diagnostic and Therapeutic Applications." Annals of Bmedical Engineering (2014). Web.

28. Dyck, P. J., E. H. Lambert, and P. C. O'brien. "Pain in Peripheral Neuropathy Related to Rate and Kind of Fiber Degeneration." Neurology 26.5 (1976): 466. Web.

29. Moc, Evans, and Jimmy Thai. "Scanning Electron Microscopy and Histology Imaging and Analysis of Decellularized Porcine Vessel." Thesis. California Polytechnic State University, 2012. Web.

30. Hoitsma, E., J.p.h. Reulen, M. De Baets, M. Drent, F. Spaans, and C.g. Faber. "Small Fiber Neuropathy: A Common and Important Clinical Disorder." Journal of the Neurological Sciences 227.1 (2004): 119-30. Web.

31. Bakkers, Mayienne, Catharina G. Faber, Janneke G.j. Hoeijmakers, Giuseppe Lauria, and Ingemar S.j. Merkies. "Small Fibers, Large Impact: Quality of Life in Small-fiber Neuropathy." Muscle \& Nerve (2013). Web.

32. Ikeda, Masayoshi, and Yoshinori Oka. "The Relationship between Nerve Conduction Velocity and Fiber Morphology during Peripheral Nerve Regeneration." Brain and Behavior 2.4 (2012): 382-90. Web.

33. Smith, Aubrey. "Characterization and Implementation of a Decellularized Porcine Vessel as a Biologic Scaffold for a Blood Vessel Mimic." MS thesis California Polytechnic State University, 2011. Web

34. "Introduction to Embedding and Specimen Orientation Techniques." Histology Course 2013-2013. Lecture \#16. Web.

35. "IHC-PARAFFIN PROTOCOL (IHC-P)." Antibodies, Proteins, Kits and Reagents for Life Sciences. Abcam. Web.

36. "Olympus Introduces BX41 Laboratory Microscope." Olympus America. Web.

37. "Haematoxylin Eosin (H\&E) Staining." Protocols Online. Web.

38. “Tutorials and Examples.” National Institute of Health - Image J. Web.

39. Boyd, I.A., Davey, M.R., "Composition of Peripheral Nerves", E\&S Livingstone, Edinburgh, 1968. 


\section{APPENDiX A - IMAgE J BASIC InSTRUCTIONS [38]}

ImageJ Basics

(Version 1.38)

ImageJ is an image analysis program that was created at the National Institutes of Health. It is in the public domain and runs on a variety of operating systems. You may download this program from the source (http://rsb.info.nih.gov/ij/). The ImageJ website has instructions for use of the program and links to useful resources.

Installing ImageJ on your PC (Windows operating system): Copy the ImageJ folder and transfer it to the $\mathrm{C}$ drive of your personal computer. Open the ImageJ folder in the $\mathrm{C}$ drive and copy the shortcut (microscope with arrow) to your computer's desktop. Double click on this desktop shortcut to run ImageJ. See the ImageJ website for Macintosh instructions.

ImageJ Window: The ImageJ window will appear on the desktop. Note that this window has a Menu Bar, a Tool Bar and a Status Bar. 

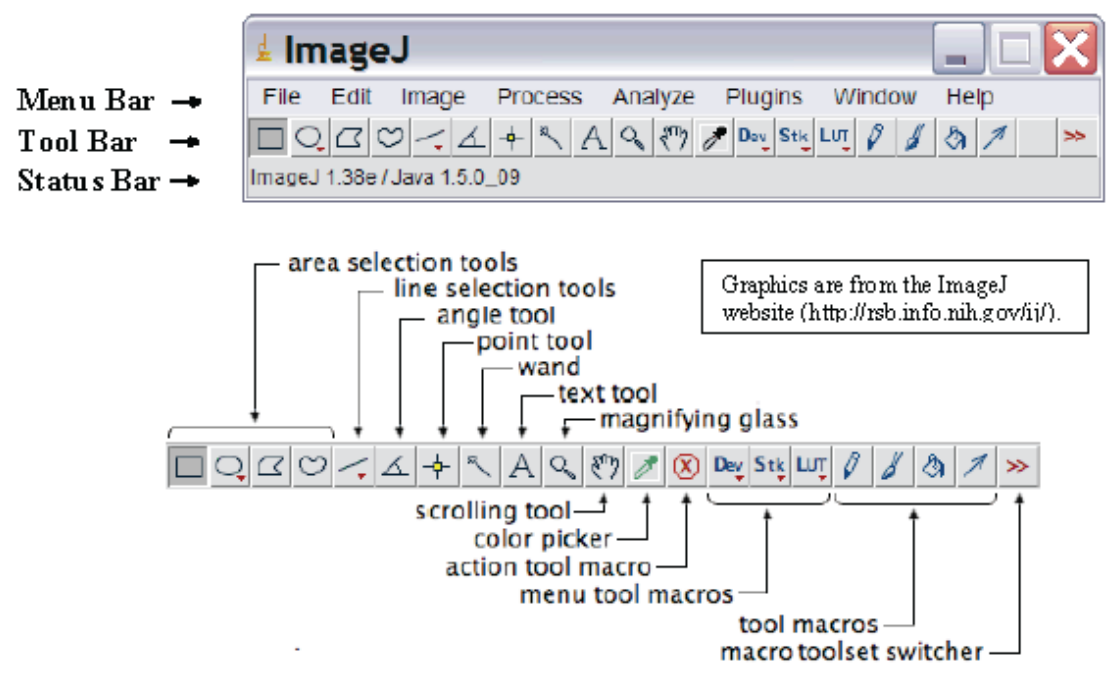

Adjusting Memory Allocation: Use the Edit $\rightarrow$ Options $\rightarrow$ Memory command to adjust the default memory allocation. Setting the maximum memory value to more than about $75 \%$ of real RAM may result in poor performance due to virtual memory paging.

Opening an Image File: Select File $\rightarrow$ Open from the menu bar to open a stored image file.

Tool Bar: The various buttons on the tool bar allow you measure, draw, label, fill, etc. A rightclick or a double left-click may expand your options with some of the tool buttons.

Area Selection Tools: The first four buttons on the tool bar allow you to surround an area on the image with a rectangle, oval, polygon or freehand shape. After selection, these areas may be altered, analyzed, copied, etc. using the menu commands. The status bar, below the tool bar, gives information such as the coordinates (xx, yy) of the selection on the frame.

Line Selection Tools: This button allows you to create straight, segmented or freehand lines (right-click to select line type). Information about the line is displayed on the status bar.

Double-click on the button to alter the line width, select Analyse $\rightarrow$ Measure (or Ctrl+M) to record a line length and $\boldsymbol{E d i t} \rightarrow$ Draw (or Ctrl+D) to make a line permanent.

Angle Tool: Draws two intersecting lines and measures the formed angle.

Point Tool: When 'Auto-Measure' is selected, this tool allows you to mark locations on an 
image; with each click the coordinates of the mark (xx, yy) and brightness values (0-255) are recorded in a data window. Color images will have three brightness readings displayed on the status bar, one each for the red, green and blue channels, however only a single grayscale brightness value will appear in the data window.

Wand Tool: This tool automatically finds the edge of an object and traces its shape. It works best with high contrast images. Place the wand to the left of an edge and click. The algorithm will search to the right for an edge. It will then trace along the edge of the object until it returns to the starting point.

Text Tool: Double click on this button to select a font and size. Single click the button, clickdrag a text box and type the label. Move the box to the desired location and permanently set the text in place with $\boldsymbol{E d i t} \rightarrow$ Draw (or Ctrl+D).

Magnifying Glass: Left-click on the image to magnify; right-click to reduce the image size.

Scrolling Tool: This button allows you to move an image if it is larger than the window.

Color Picker: This tool sets the foreground drawing/text color when a color on an image is 'picked up’ with the eyedropper. Colors also may be selected from the Colors window by double-clicking the color picker button (or via Image $\rightarrow$ Color $\rightarrow$ Color Picker). Use Alt+click to change the background color. The icon for this tool (eye dropper) shows the current foreground color while the frame around it shows the background color.

Other Tools: The remaining tool buttons are similar to those found in drawing programs (spray can, flood fill, etc.) and can be easily used after a bit of experimentation.

\section{Image Editing and Analysis}

The following is a small sampling of processing techniques that are possible with ImageJ. It is common to use ImageJ in conjunction with a photo editing program such as Adobe Photoshop. During analysis, measured values can be easily transferred to a spreadsheet with a 'cut and paste'. Refer to the ImageJ and NIH Image websites for more information 
Undo. $\quad \boldsymbol{E}$ dit $\rightarrow$ Undo reverses the preceding action. Only one back-step is possible.

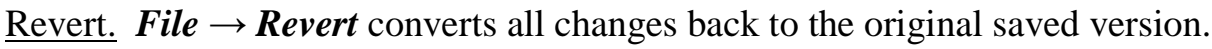

Cropping. Surround the area with the rectangular selection tool followed by Image $\rightarrow$ Crop.

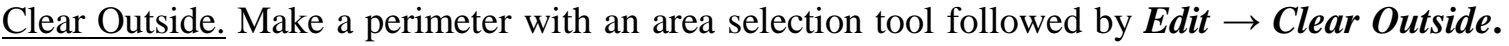
This technique is useful for clearing extraneous objects near an area of interest. Edit $\rightarrow$ Clear clears inside of the perimeter.

Enhancing Brightness and Contrast. Image $\rightarrow$ Adjust $\rightarrow$ Brightness/Contrast; click 'Auto' or set manually with the sliders. Also try Process $\rightarrow$ Enhance Contrast.

Removing Noise. Process $\rightarrow$ Noise $\rightarrow$ Despeckle or try Process $\rightarrow$ Filters $\rightarrow$ Median

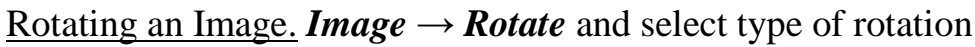

Converting to Grayscale. Image $\rightarrow$ Type $\rightarrow$ 8-bit converts the image to 256 shades (8-bit) of gray. In this scale $0=$ pure black and 255 = pure white.... a grayscale reading of 128 would be a medium gray.

Thresholding (Binary Contrast Enhancement). This is commonly used when detecting edges, counting particles or measuring areas. A grayscale image is converted to binary (a.k.a. halftone or black \& white) by defining a grayscale cutoff point. Grayscale values below the cutoff become black and those above become white. The procedure: First convert the image to 8-bit grayscale as described above (Image $\rightarrow$ Type $\rightarrow$ 8-bit). Create a 'thresholded' binary image by selecting Process $\rightarrow$ Binary $\rightarrow$ Make Binary. A less automated procedure involves: Image $\rightarrow$ Adjust $\rightarrow$ Threshold; use the slider to adjust the threshold. The red areas will become the black portions in the binary image. Click 'Apply' to complete the conversion. 'Brightness slicing' is a similar procedure that uses both upper and lower threshold values.

\section{Measuring and Counting Objects}

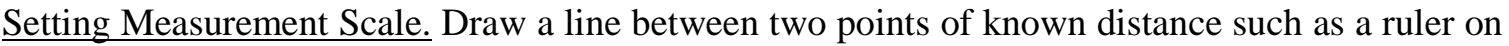
the photograph. Go to Analyze $\rightarrow$ Set Scale. In the Set Scale window the length of the line, in 
pixels, will be displayed. Type the known distance and units of measure in the appropriate boxes and click OK. Measurements will now be shown using these settings. If the pixel:length relationship is known from a previous measurement you may directly type this information in the Set Scale window. Check 'global' to apply this scale to other image frames.

$\underline{\text { Set Measurements. }}$. Choose parameters to be measured via Analyze $\rightarrow$ Set Measurements.

Measuring Distance Between Points. Draw a line between two points. The status bar will show the angle (from horizontal) and the length. Analyze $\rightarrow$ Measure (or Ctrl+M or simply type $\mathrm{M}$ on the keyboard) transfers the values to a data window.

Measuring Area. Surround an area with a perimeter. This can be done with an area selection tool, the wand (for high contrast images) or with Analyze Particles (see below). Analyze $\rightarrow$ Measure (or keyboard M) transfers the area measurement to a data window.

Counting Particles. As described above, convert the image to 8-bit grayscale and then 'threshold' the image. Go to Analyze $\rightarrow$ Analyze Particles, type the upper and lower limits for the particle size, toggle 'show outlines' and check 'Display Results'. Click OK and each counted particle will be outlined and numbered in a new widow (numbers may be very small). The data window contains measurements for each particle.

Saving Files. Images from digital cameras are usually saved as JPEG files. JPEG is a type of memory compression that results in the loss of some data. A JPEG image degrades each time it is opened, edited and resaved. It is best to save a file in a 'lossless' format such as a TIFF during the editing process (File $\rightarrow$ Save As $\rightarrow$ Tiff).

Printing. Should you encounter printing problems, save the processed image and print with a photo editor. Saved images also may be inserted into MS Word for printing. 


\section{APPENDIX B - H \& E STAINED IMAGES}

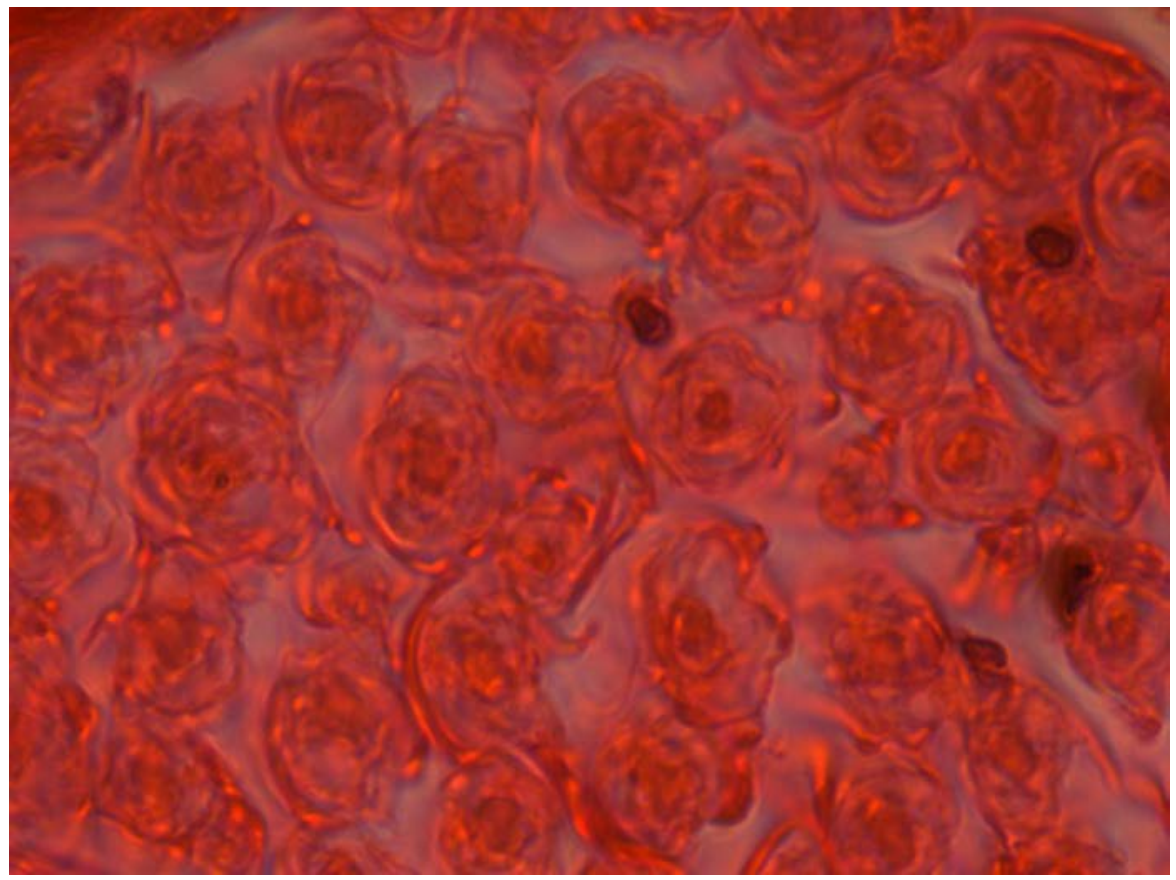

Figure 43: Block 12-028, Slide 1, A

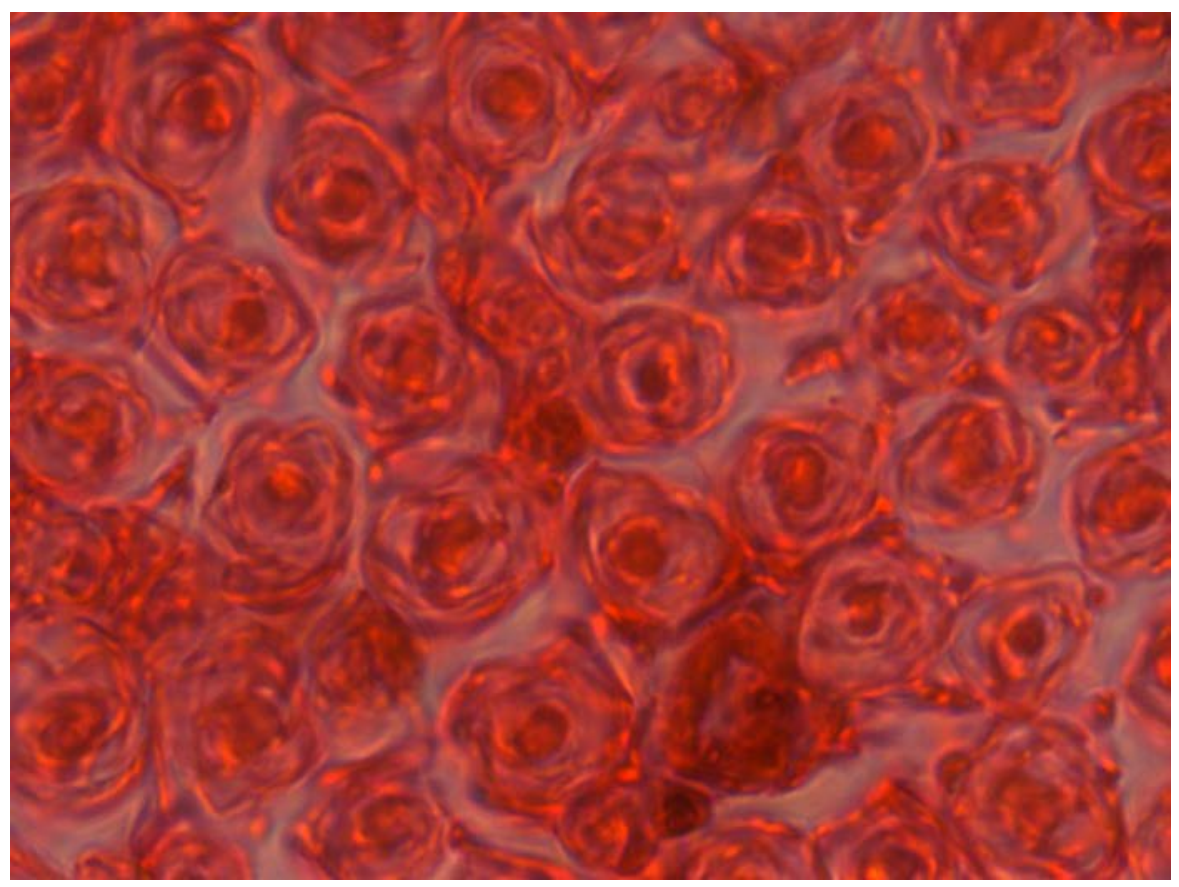

Figure 44: Block 12-028, Slide 1, B 


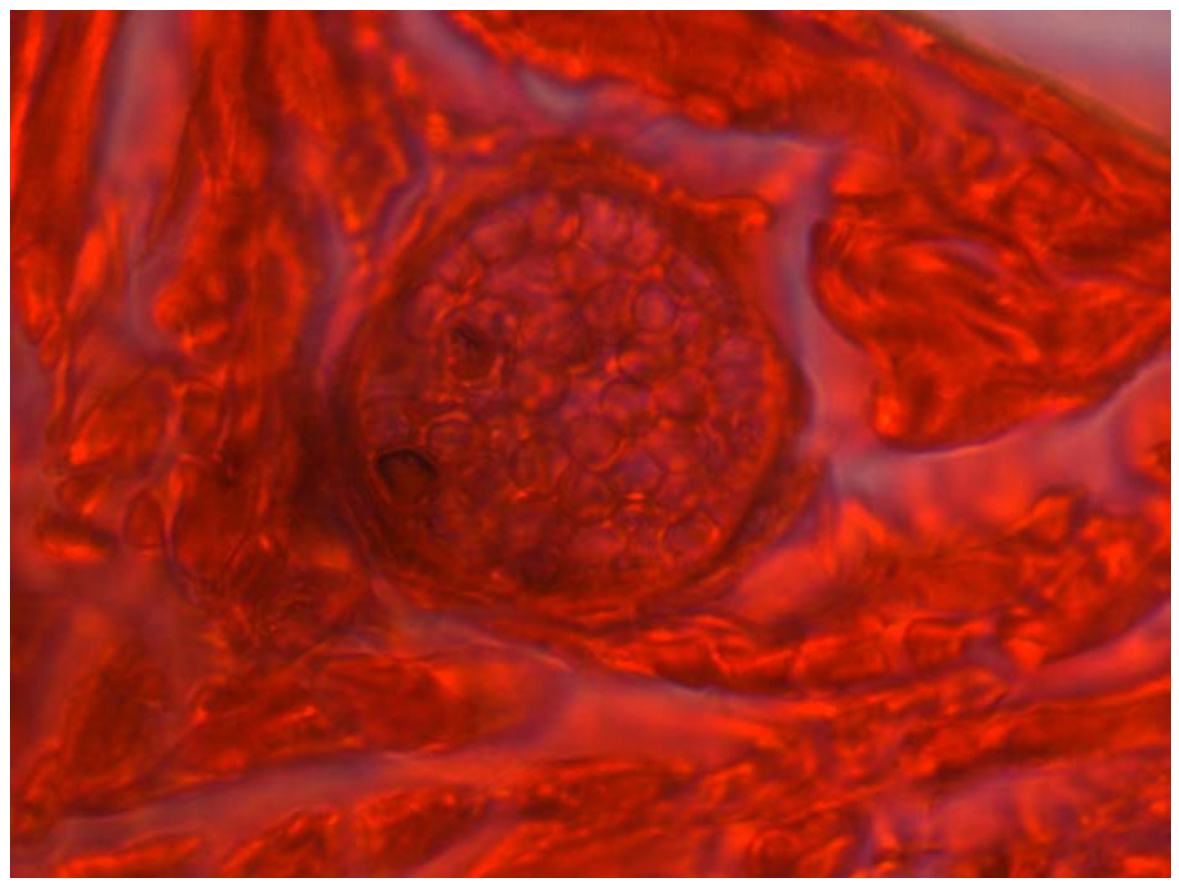

Figure 45: Block 12-028, Slide 2, A:

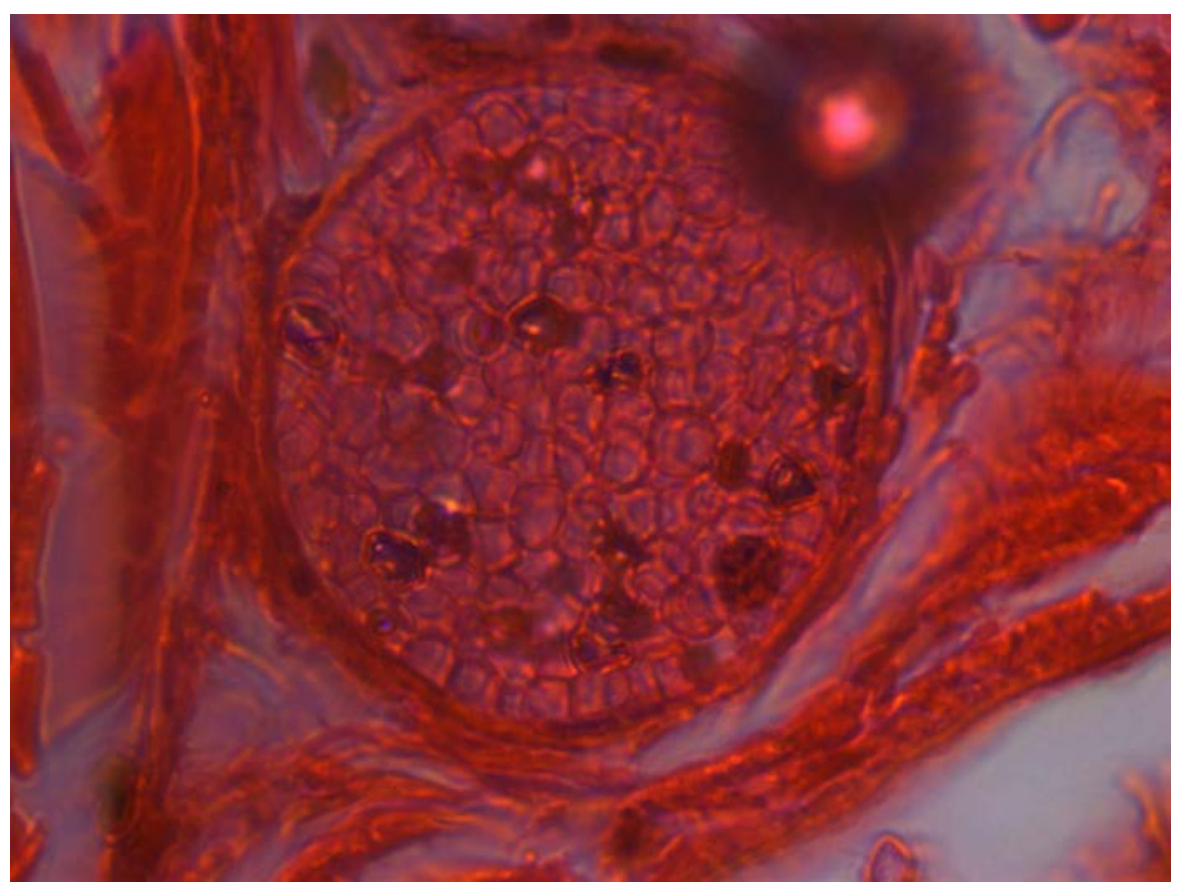

Figure 46: Block 12-028, Slide 2, B 


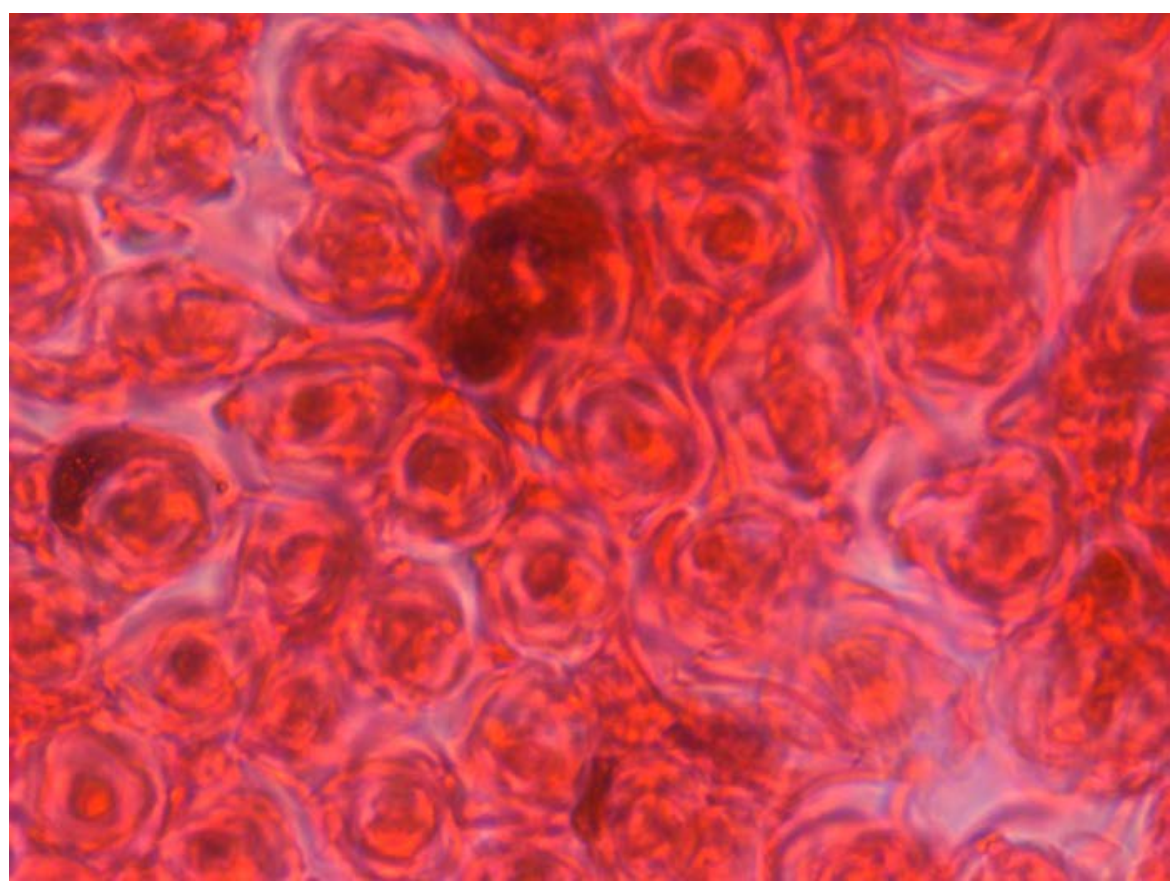

Figure 47: Block 12-028, Slide 3, A

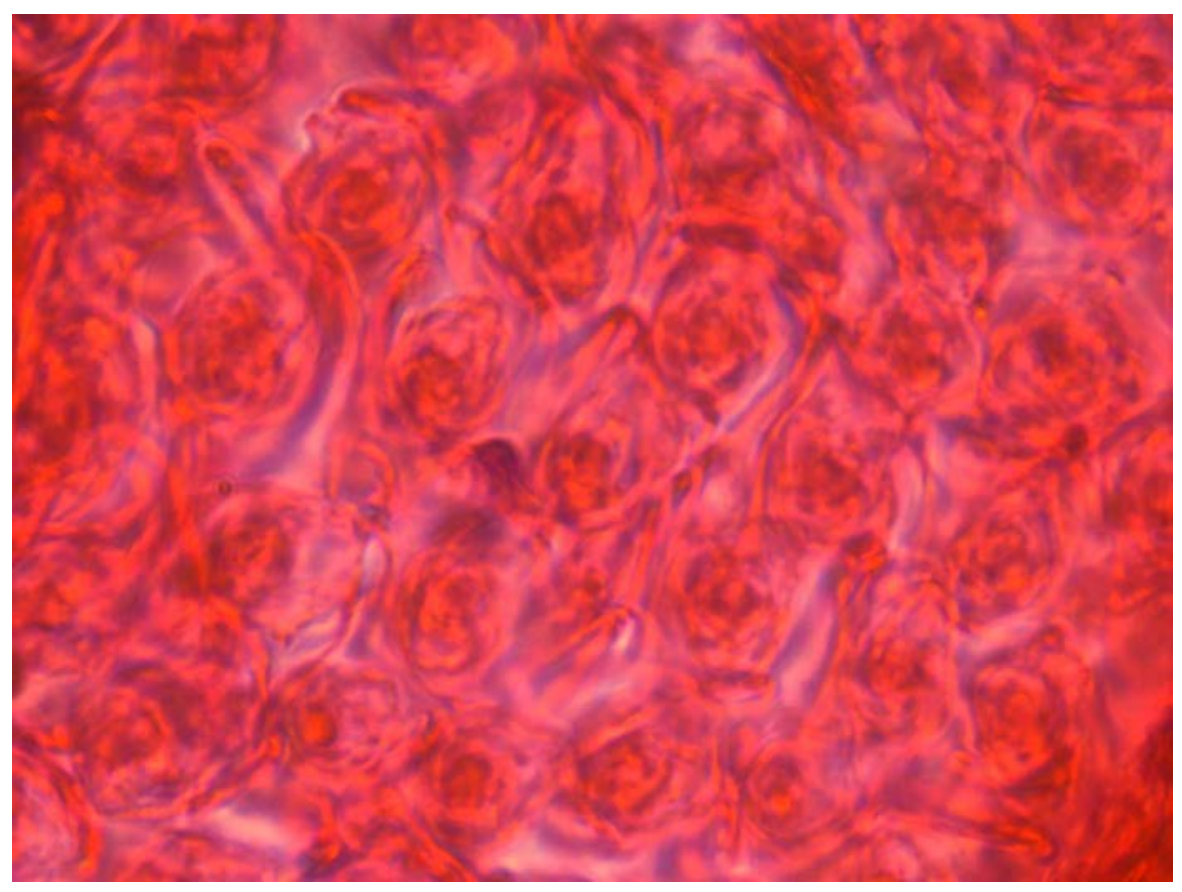

Figure 48: Block 12-028, Slide 3, B 


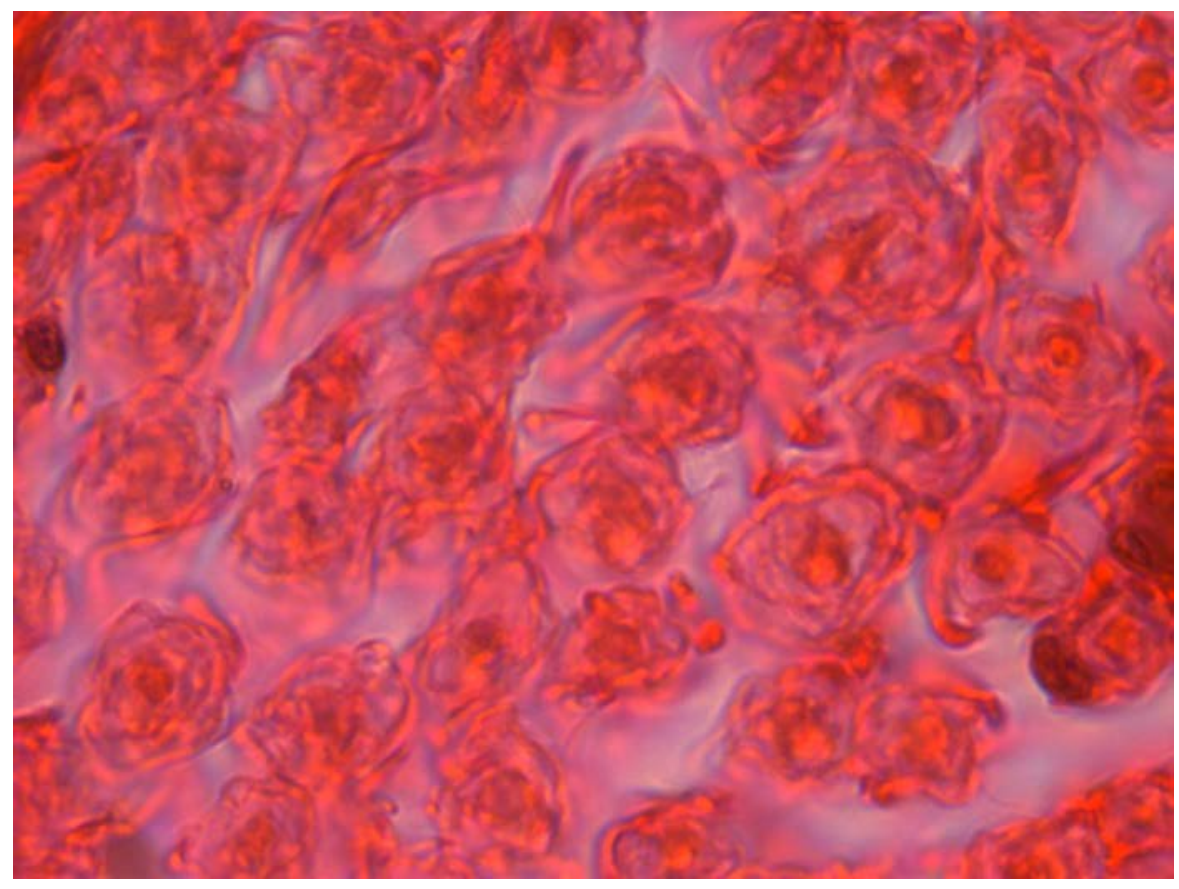

Figure 49: Block 12-028, Slide 4, A 


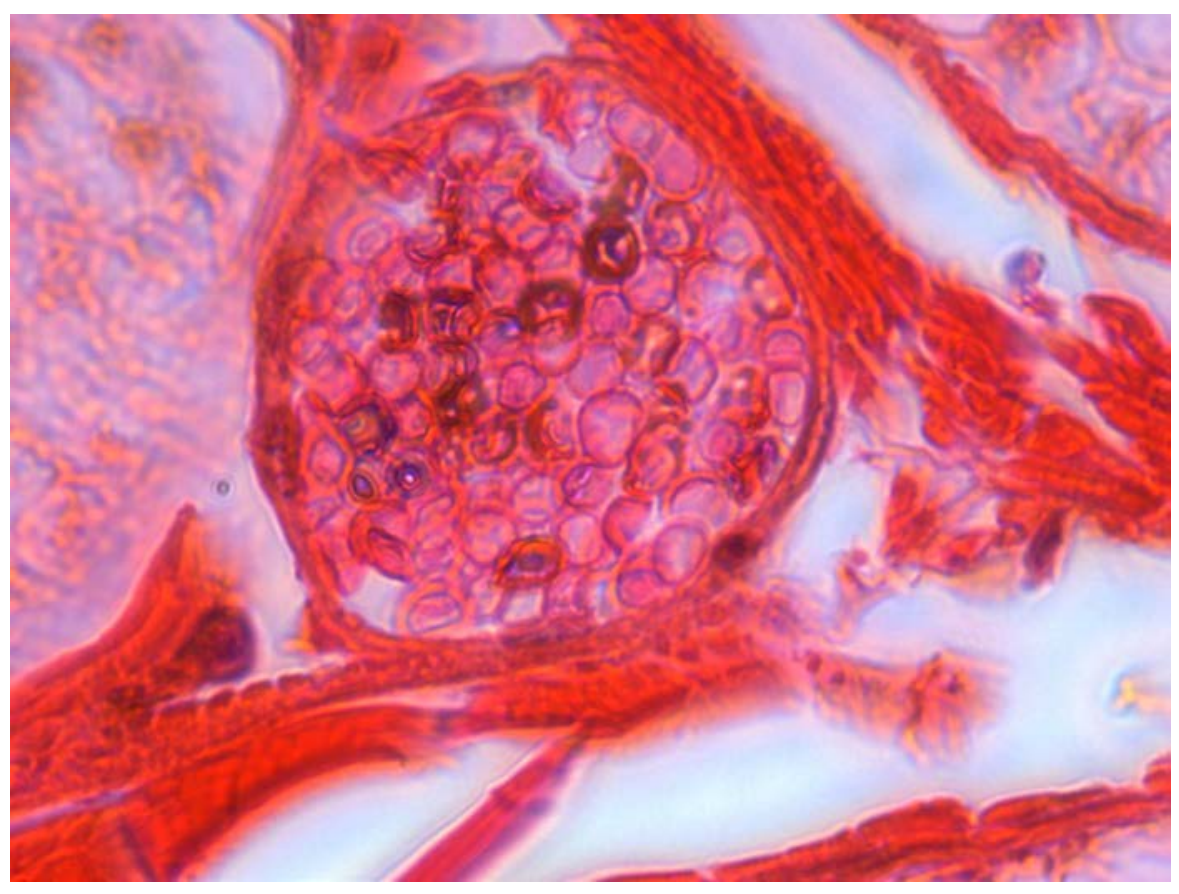

Figure 50: Block 12-028, Slide 5, A

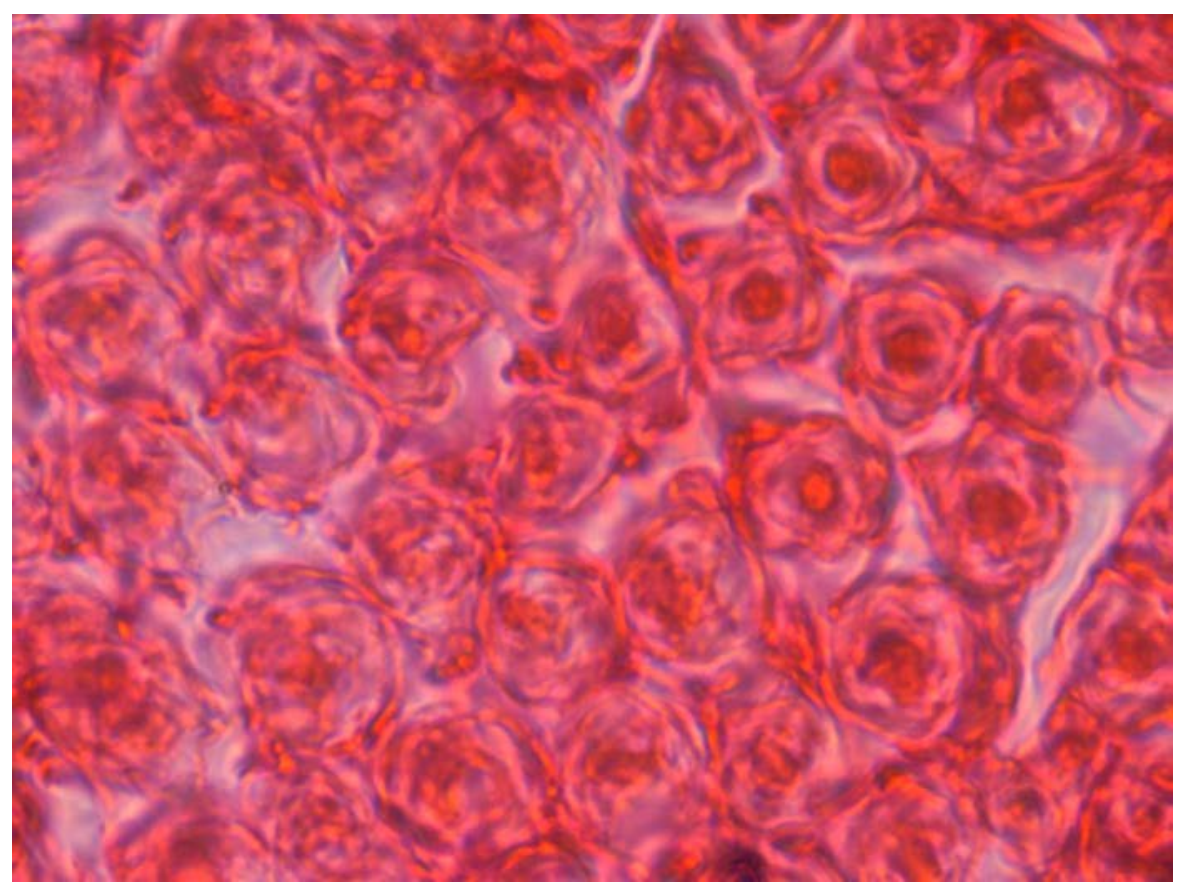

Figure 51: Block 12-028, Slide 5, B 


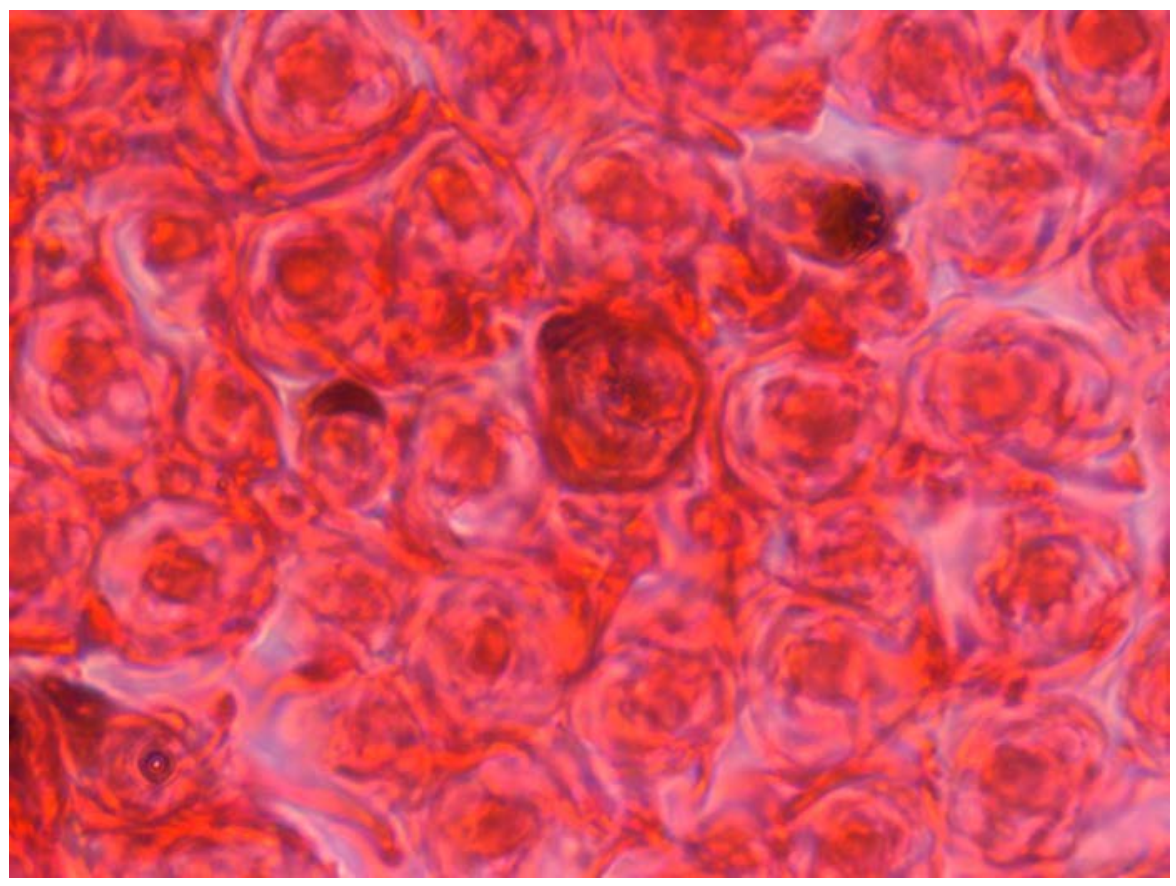

Figure 52: Block 12-029, Slide 1, A

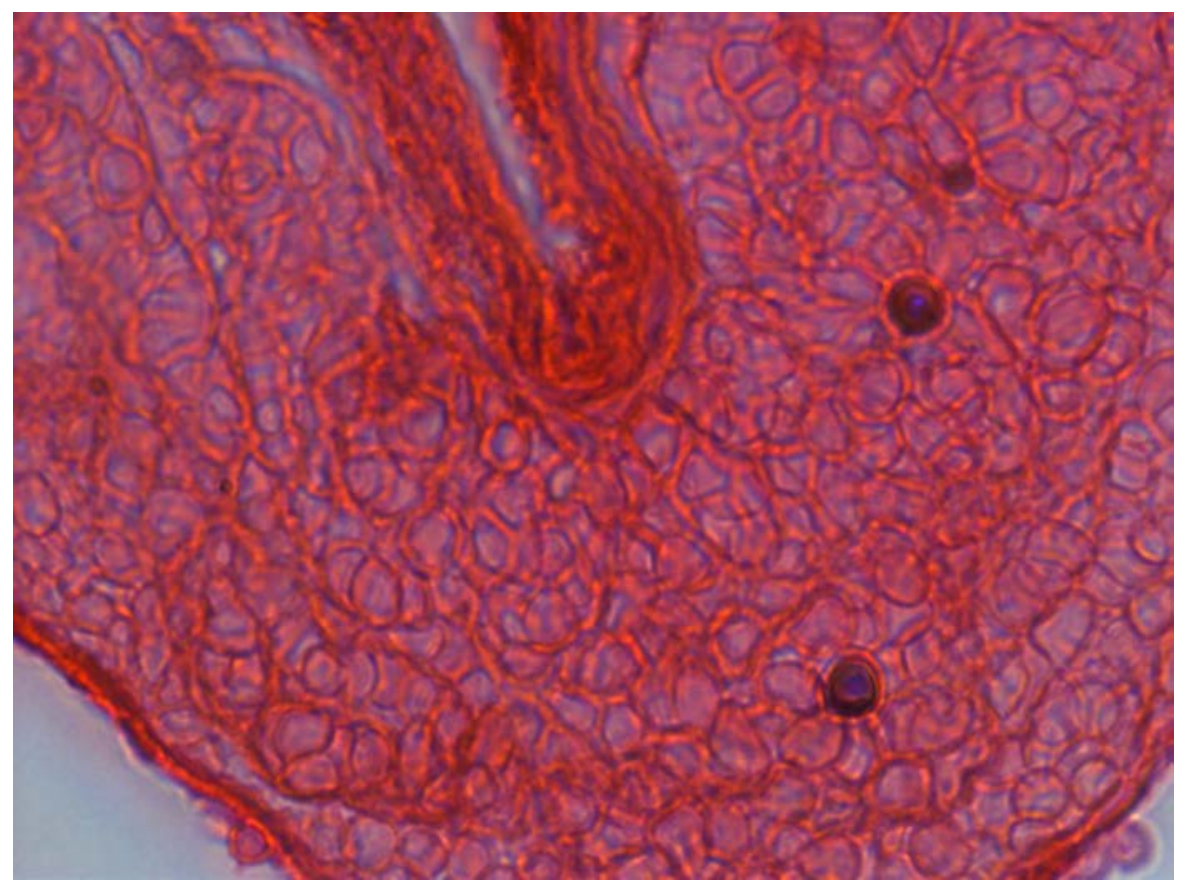

Figure 53: Block 12-029, Slide 1, B 


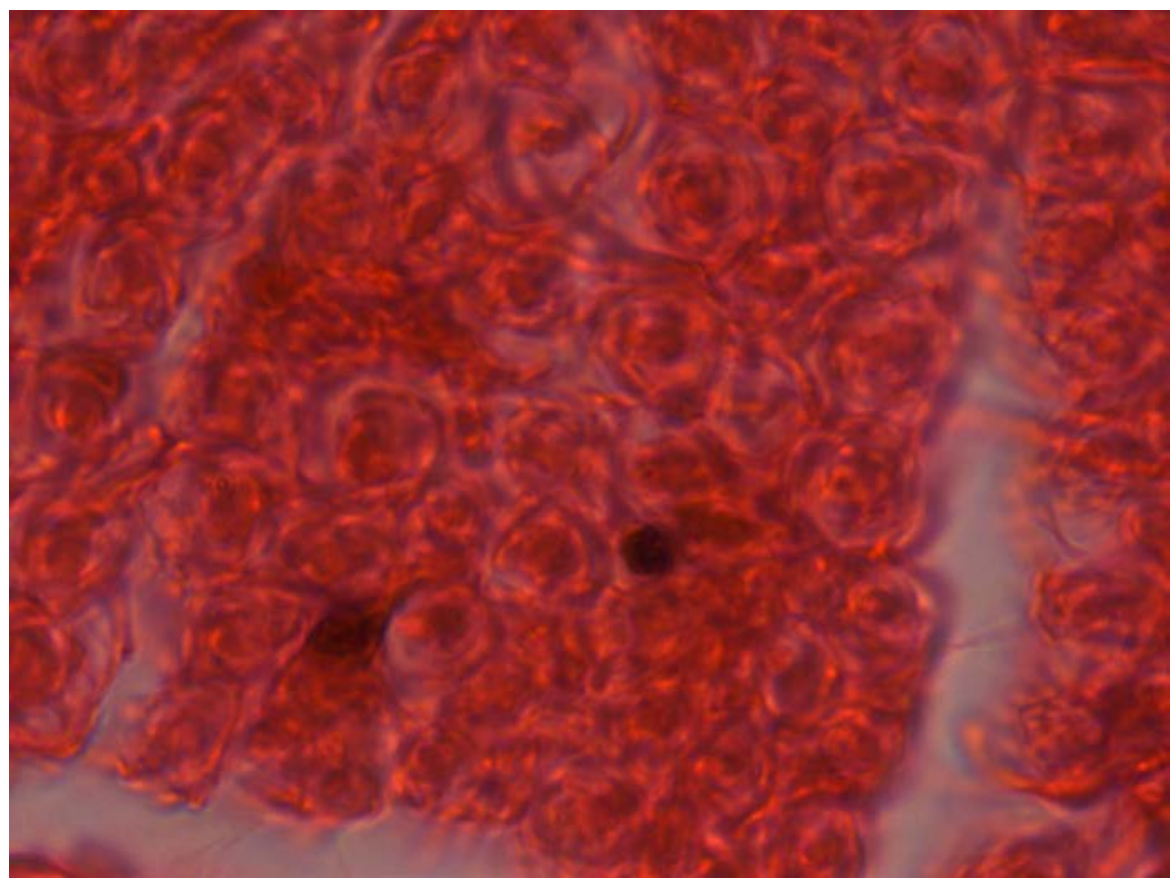

Figure 54: Block 12-029, Slide 2, A 


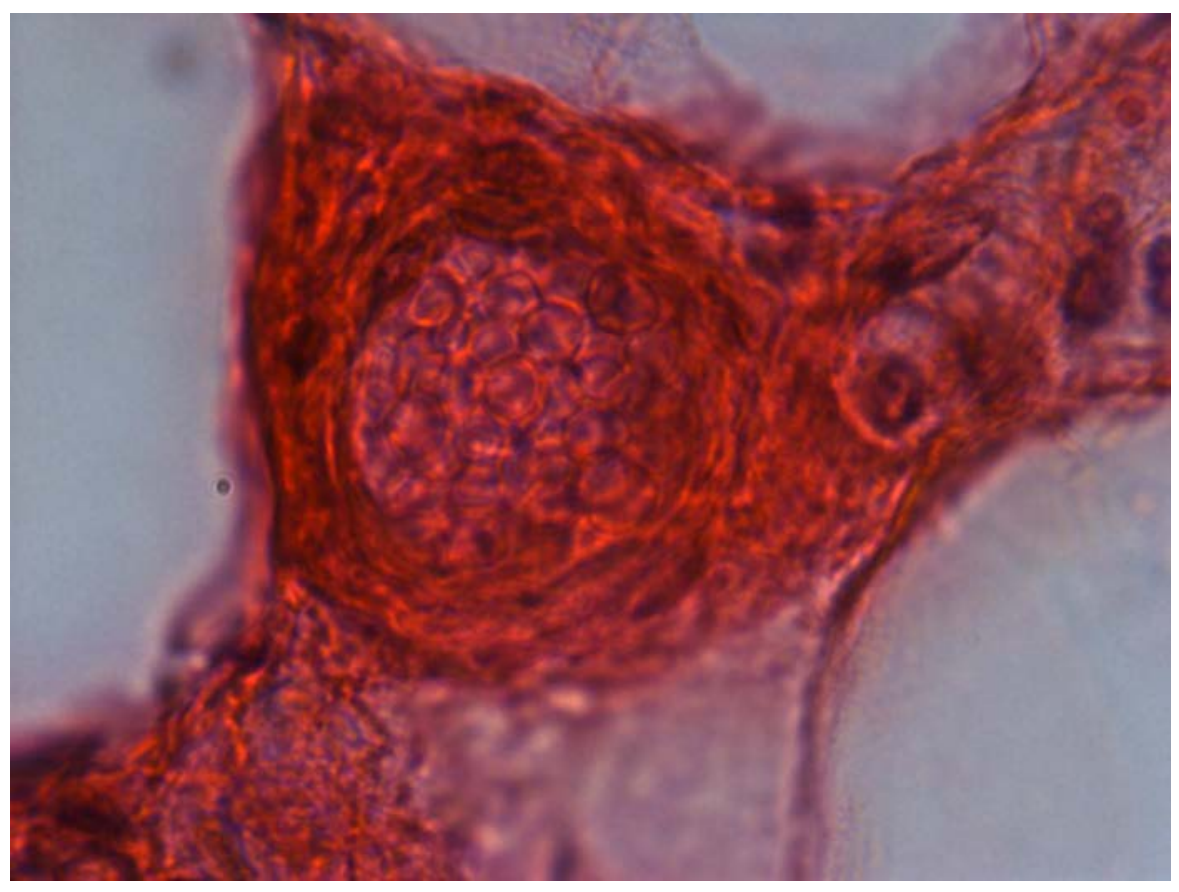

Figure 55: Block 12-029, Slide 4, A 


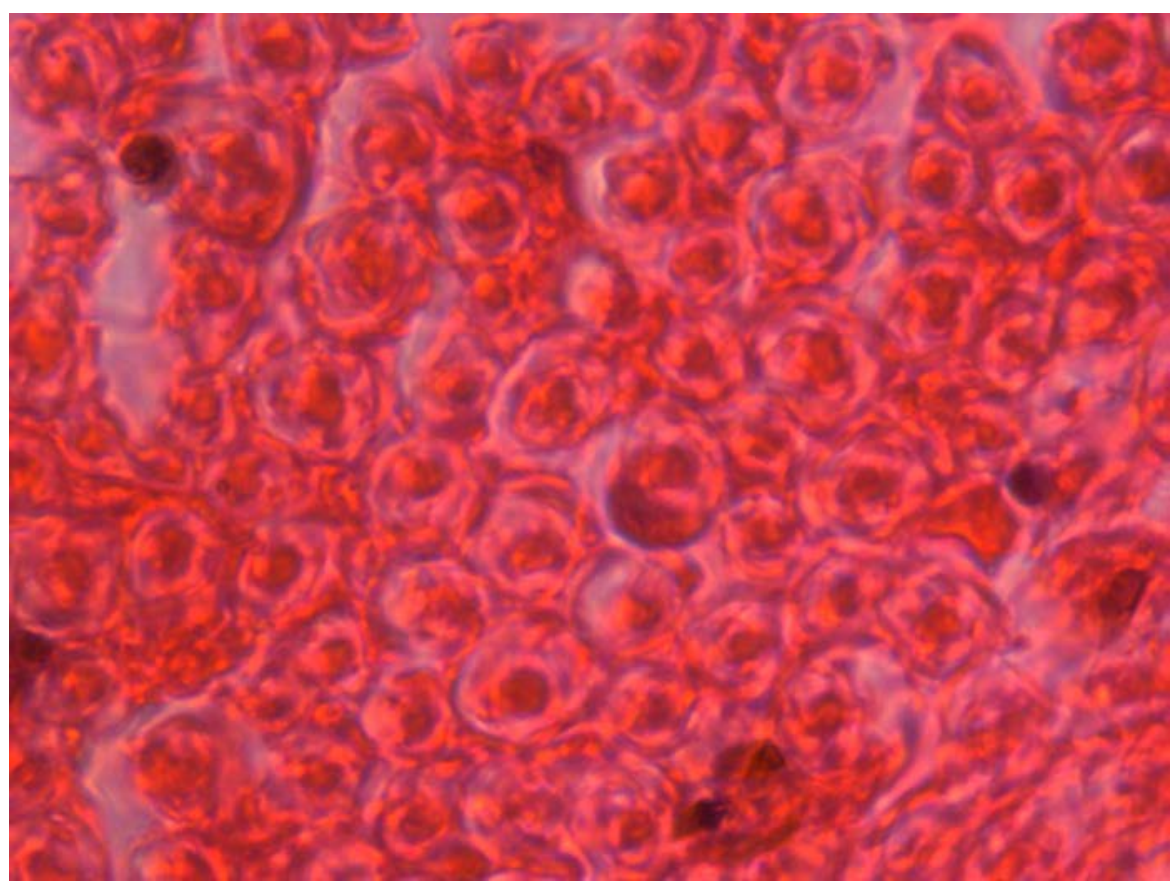

Figure 56: Block 12-029, Slide 5, A

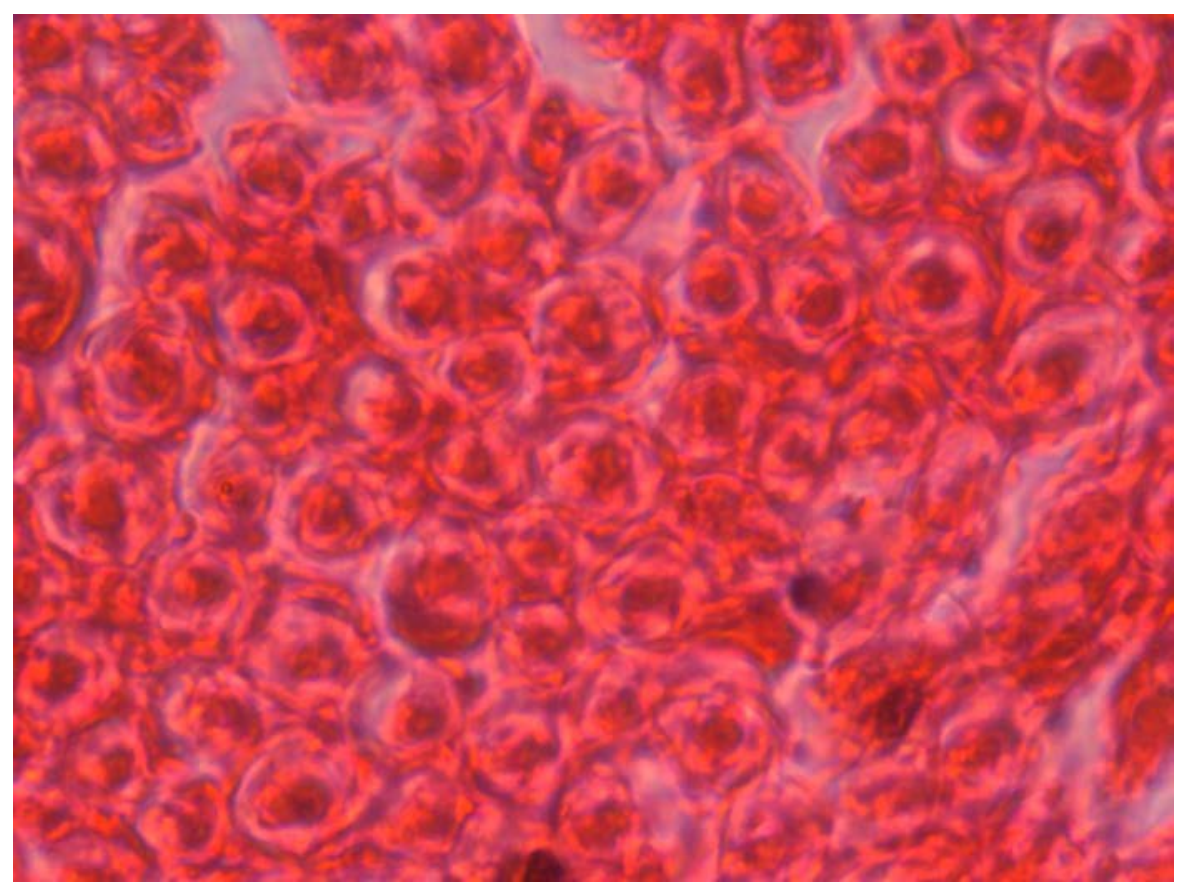

Figure 57: Block 12-029, Slide 5, B 


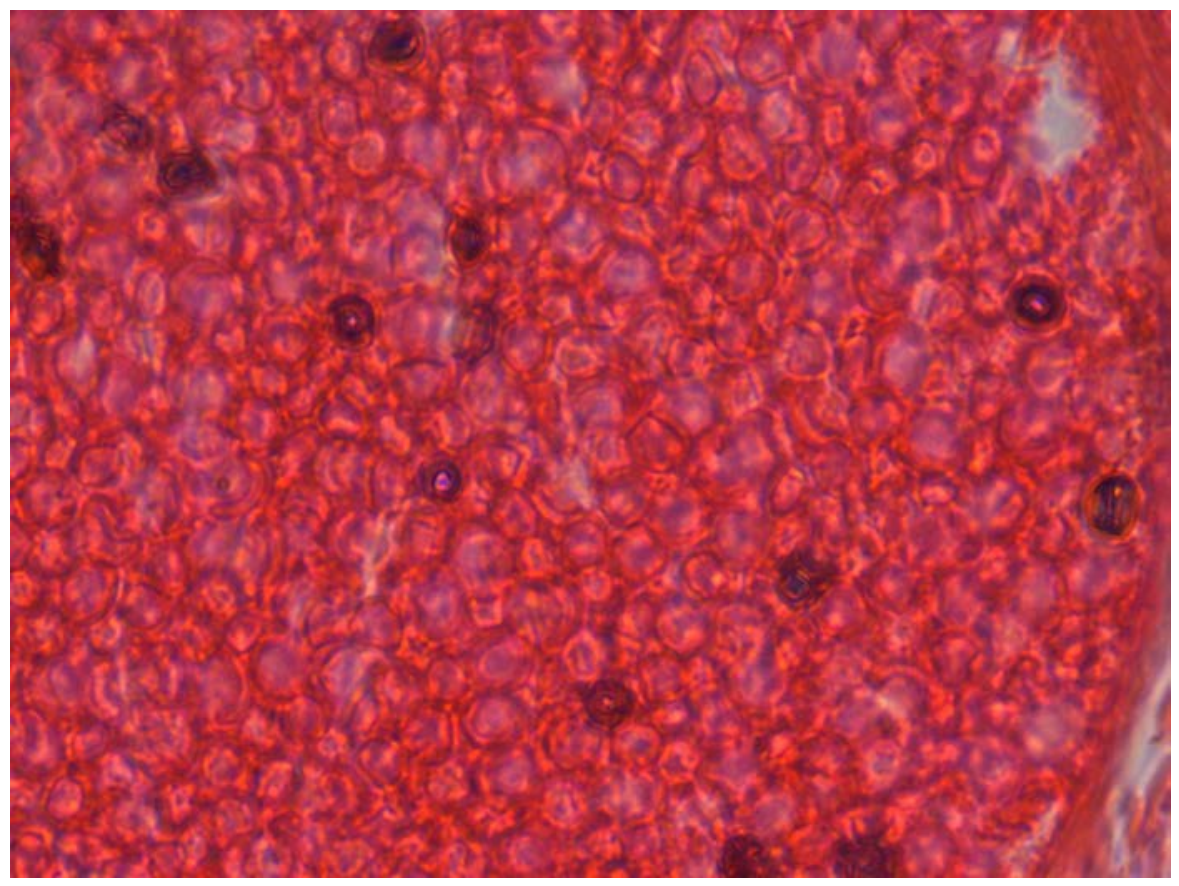

Figure 58: Block 12-030, Slide 1, A

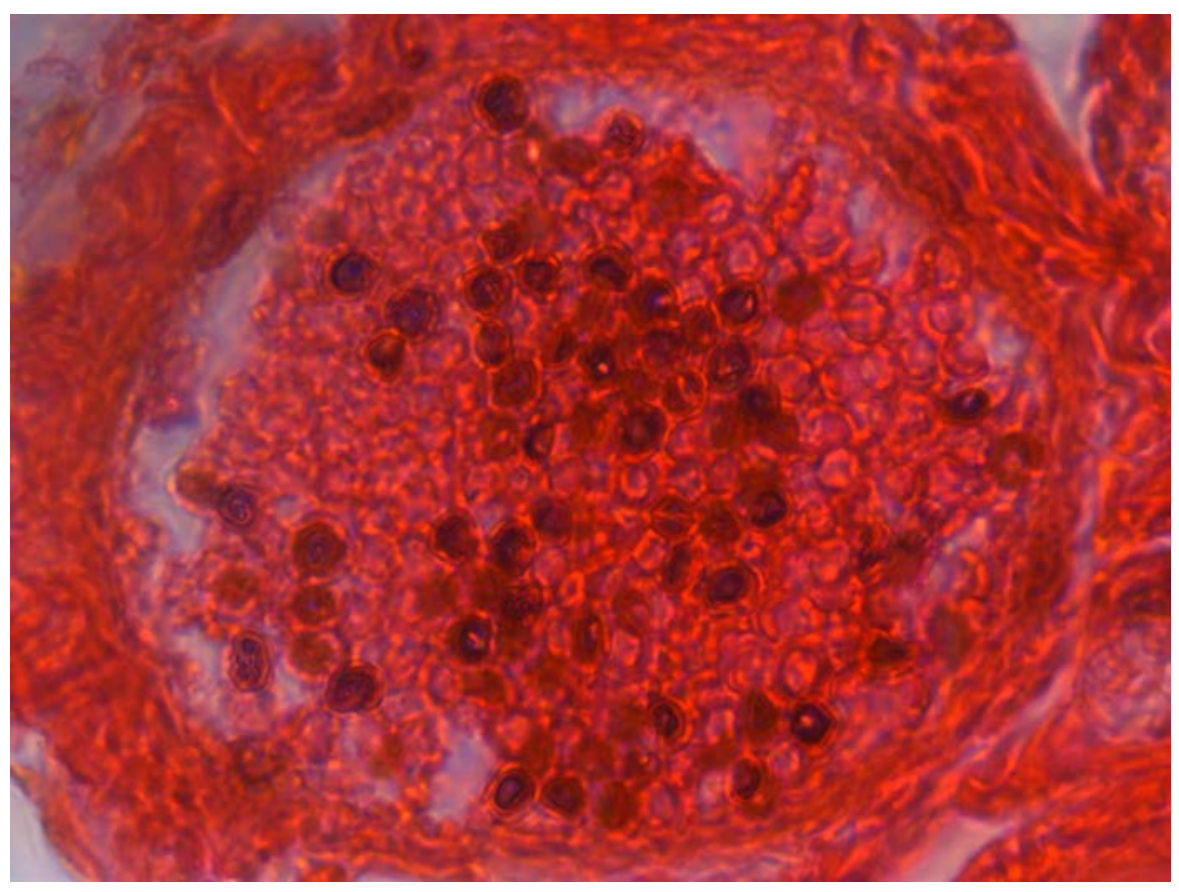

Figure 59: Block 12-030, Slide 1, B 


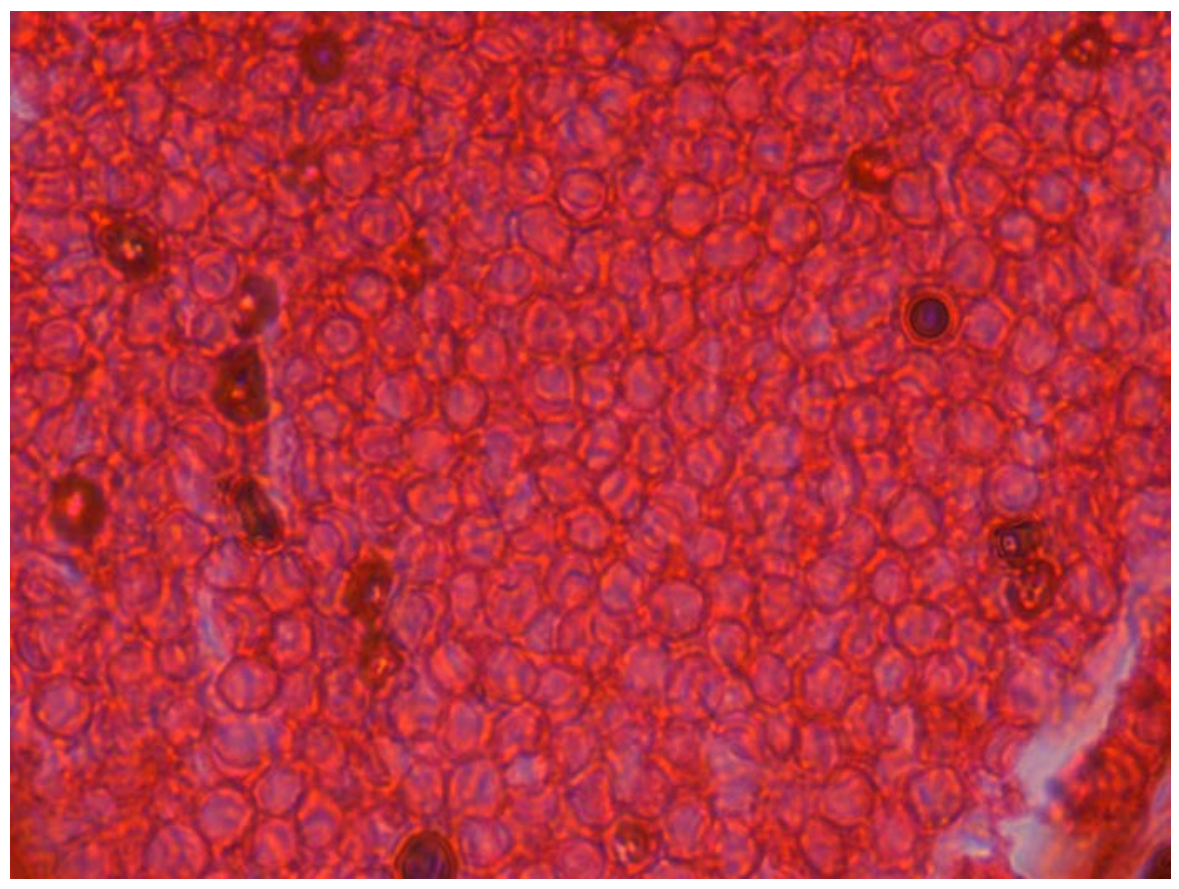

Figure 60: Block 12-030, Slide 2, A 


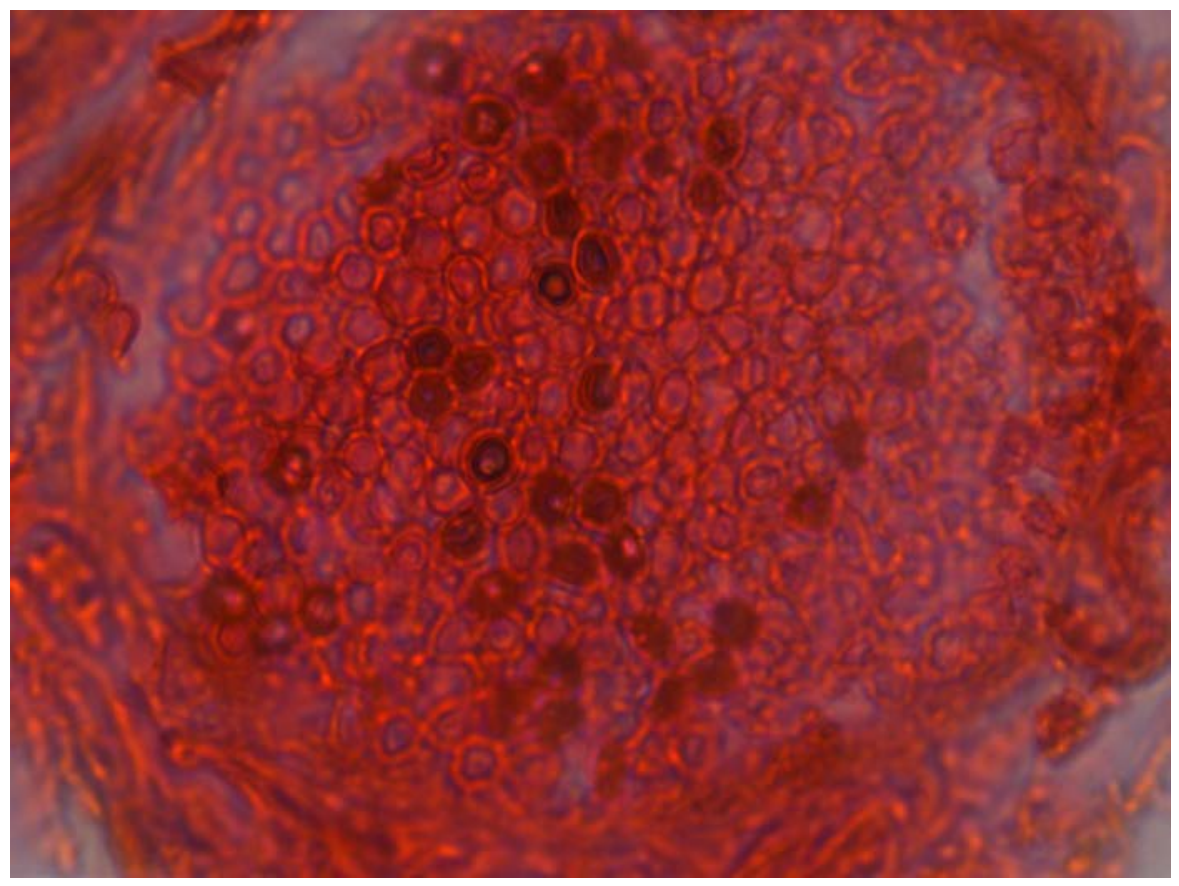

Figure 61: Block 12-030, Slide 3, A

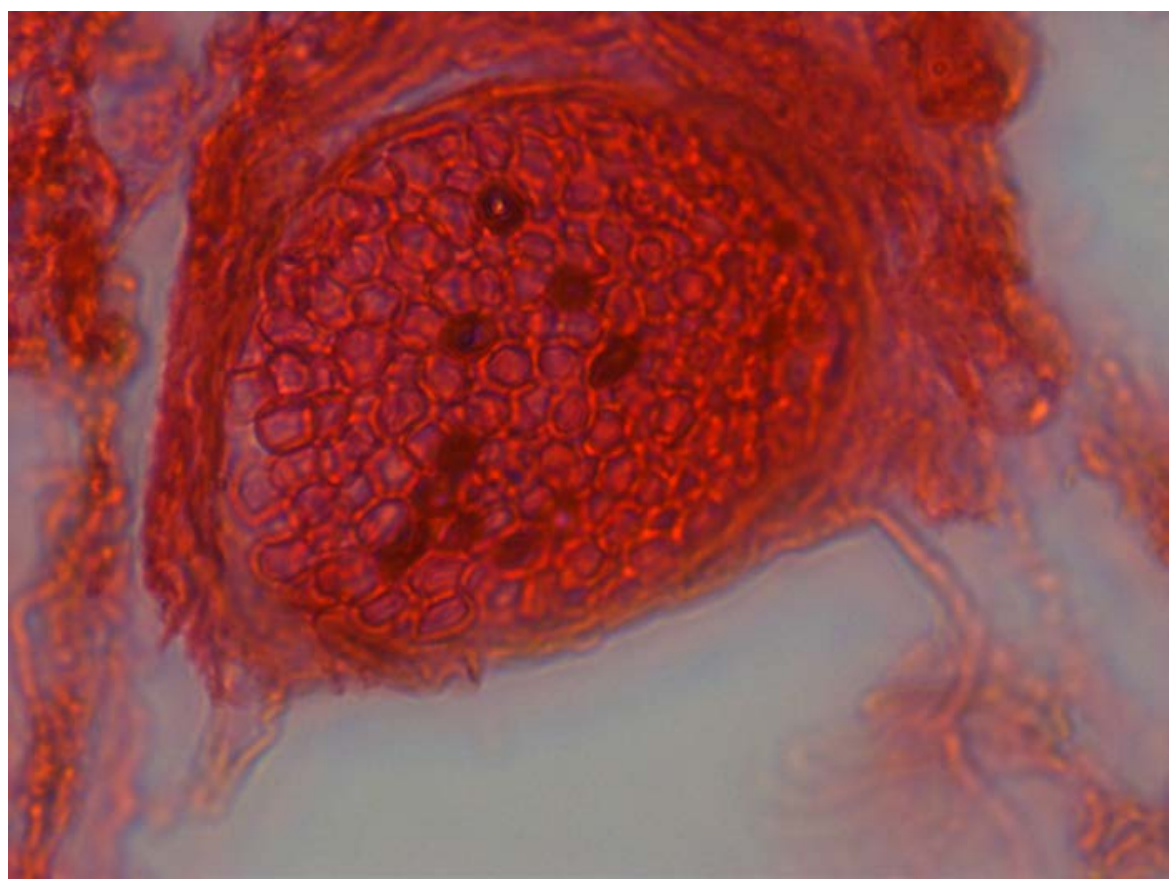

Figure 62: Block 12-030, Slide 3, B 


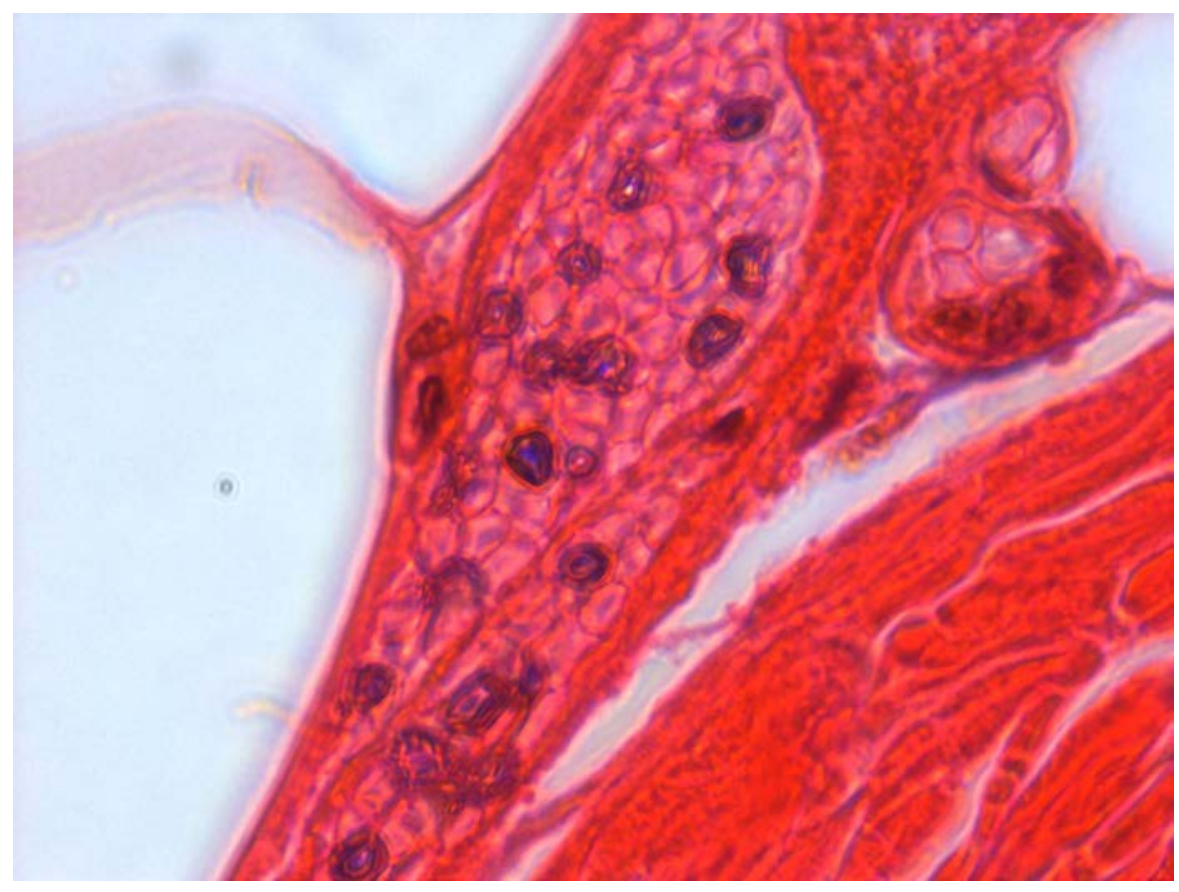

Figure 63: Block 12-030, Slide 4, A 


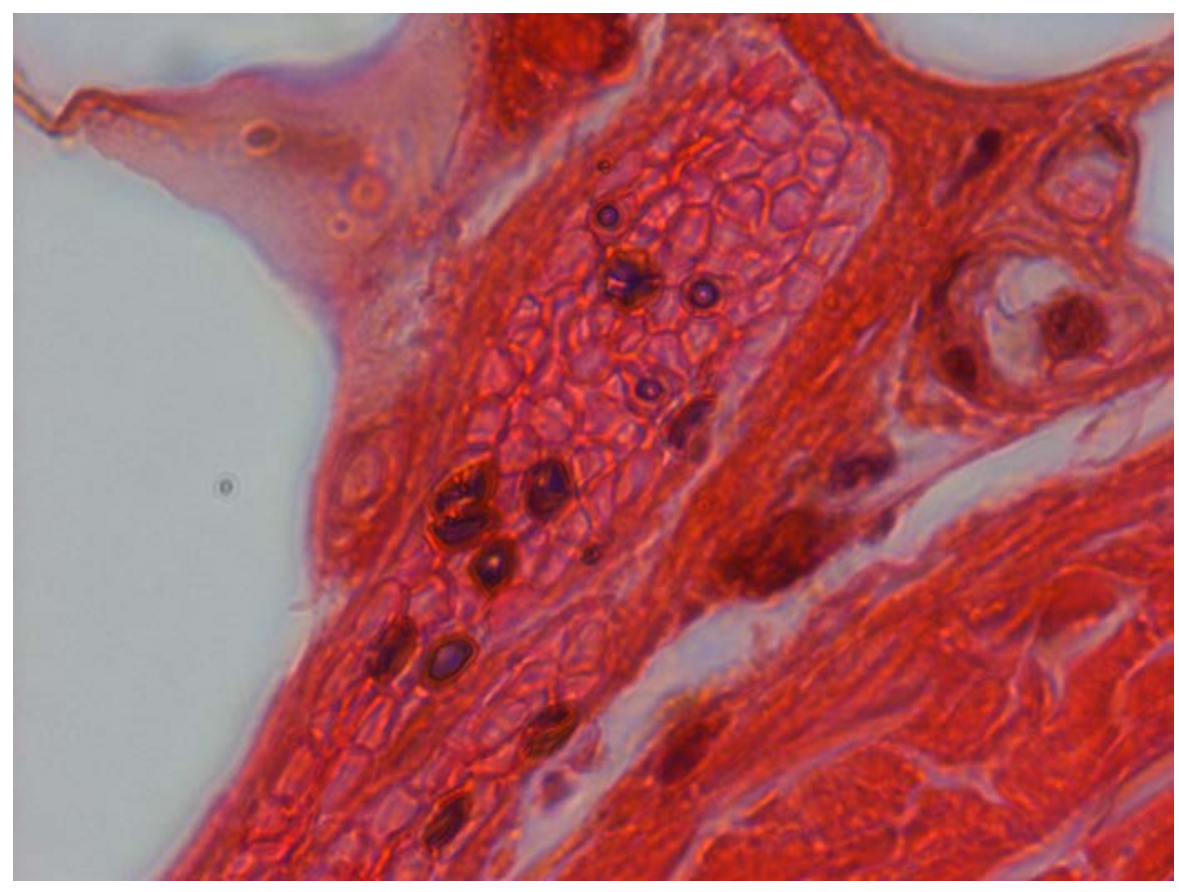

Figure 64: Block 12-030, Slide 5, A 


\section{Appendix C - RAw Measurement Data}

Sample: 12.028.1.1

\begin{tabular}{rrrrrrrrr}
\multicolumn{1}{l}{ Area } & \multicolumn{1}{c}{ Mean } & \multicolumn{1}{c}{ Min } & Max & Perim. & Diam (using A) & Diam (using P) & Average D \\
1 & 79.8541 & 81.903 & 60 & 110 & 33.6022 & 10.08332276 & 10.69591246 & 10.38962 \\
2 & 85.5443 & 82.2456 & 59 & 118 & 33.9566 & 10.43639716 & 10.80872148 & 10.62256 \\
3 & 90.0802 & 85.6158 & 63 & 120 & 35.4999 & 10.70951319 & 11.29996913 & 11.00474 \\
4 & 77.2366 & 82.0626 & 58 & 114 & 31.7468 & 9.916687623 & 10.10532029 & 10.011 \\
5 & 73.7737 & 83.6054 & 57 & 108 & 32.011 & 9.69183121 & 10.18941777 & 9.940624 \\
6 & 86.0117 & 75.5752 & 57 & 116 & 34.2915 & 10.4648697 & 10.91532346 & 10.6901 \\
7 & 64.901 & 86.4719 & 61 & 109 & 29.8751 & 9.090353112 & 9.509539681 & 9.299946 \\
8 & 73.9322 & 82.744 & 62 & 116 & 33.2643 & 9.70223689 & 10.58835555 & 10.1453 \\
9 & 66.1569 & 85.0985 & 61 & 107 & 29.8197 & 9.177885445 & 9.491905313 & 9.334895 \\
10 & 61.8039 & 85.5589 & 65 & 116 & 30.1617 & 8.870804332 & 9.600767294 & 9.235786 \\
11 & 88.0439 & 85.3444 & 61 & 117 & 34.5828 & 10.5877748 & 11.00804713 & 10.79791 \\
12 & 66.9292 & 82.4189 & 47 & 120 & 30.2748 & 9.231300241 & 9.636768142 & 9.434034 \\
13 & 95.1974 & 84.0054 & 56 & 111 & 36.4994 & 11.00950018 & 11.61811986 & 11.31381 \\
14 & 56.0649 & 83.2074 & 61 & 105 & 27.401 & 8.44890808 & 8.722009191 & 8.585459 \\
15 & 20.1109 & 81.002 & 57 & 104 & 16.8972 & 5.060236473 & 5.378545809 & 5.219391 \\
16 & 40.4006 & 80.6848 & 49 & 111 & 24.1462 & 7.172143442 & 7.685974174 & 7.429059 \\
17 & 36.7222 & 84.8393 & 60 & 111 & 24.0899 & 6.837847411 & 7.668053327 & 7.25295 \\
18 & 79.1794 & 85.8157 & 59 & 114 & 34.3363 & 10.0406346 & 10.92958374 & 10.48511 \\
19 & 56.5526 & 83.5753 & 62 & 109 & 28.2064 & 8.48557639 & 8.978375974 & 8.731976 \\
20 & 84.5363 & 87.2615 & 59 & 115 & 36.1144 & 10.37472699 & 11.49557055 & 10.93515 \\
21 & 42.8921 & 78.9782 & 50 & 115 & 25.2809 & 7.389987678 & 8.047160402 & 7.718574 \\
22 & 66.3927 & 83.9333 & 59 & 117 & 30.4613 & 9.194227054 & 9.696132936 & 9.44518 \\
23 & 52.3297 & 81.4141 & 62 & 114 & 26.699 & 8.162612535 & 8.498555651 & 8.330584 \\
24 & 58.4589 & 82.3681 & 56 & 114 & 28.1462 & 8.627408836 & 8.959213719 & 8.793311 \\
25 & 52.4923 & 83.916 & 58 & 120 & 27.0442 & 8.175284225 & 8.608436224 & 8.39186 \\
26 & 85.7272 & 86.8515 & 65 & 116 & 34.2283 & 10.44754809 & 10.89520628 & 10.67138 \\
27 & 55.0122 & 81.7395 & 60 & 112 & 27.1527 & 8.369211939 & 8.642972847 & 8.506092
\end{tabular}


Sample: 12.028.1.2

\begin{tabular}{|c|c|c|c|c|c|c|c|c|}
\hline & Area & Mean & Min & Max & Perim. & Diam (using A) & Diam (using P) & Average D \\
\hline 1 & 82.6951 & 85.3681 & 53 & 127 & 33.2392 & 10.26112 & 10.58037 & 10.42075 \\
\hline 2 & 61.2146 & 88.7193 & 61 & 124 & 28.5249 & 8.828411 & 9.079758 & 8.954085 \\
\hline 3 & 16.2943 & 85.1462 & 68 & 105 & 14.9982 & 4.554838 & 4.774075 & 4.664457 \\
\hline 4 & 42.2336 & 84.005 & 55 & 112 & 23.952 & 7.333041 & 7.624158 & 7.4786 \\
\hline 5 & 66.6203 & 88.8733 & 63 & 132 & 29.6858 & 9.209973 & 9.449284 & 9.329628 \\
\hline 6 & 53.423 & 84.5133 & 59 & 134 & 28.2725 & 8.247441 & 8.999416 & 8.623428 \\
\hline 7 & 58.4589 & 86.7697 & 62 & 122 & 28.1486 & 8.627409 & 8.959978 & 8.793693 \\
\hline 8 & 76.0742 & 84.6863 & 54 & 129 & 32.8502 & 9.841782 & 10.45654 & 10.14916 \\
\hline 9 & 90.9622 & 89.5311 & 61 & 132 & 36.1242 & 10.76182 & 11.49869 & 11.13025 \\
\hline 10 & 64.901 & 88.5487 & 58 & 134 & 30.3436 & 9.090353 & 9.658668 & 9.37451 \\
\hline 11 & 38.6203 & 85.7664 & 56 & 124 & 23.3002 & 7.012339 & 7.416684 & 7.214511 \\
\hline 12 & 39.3722 & 83.6652 & 62 & 129 & 25.4654 & 7.080271 & 8.105889 & 7.59308 \\
\hline 13 & 91.0191 & 89.5551 & 52 & 133 & 35.4424 & 10.76518 & 11.28167 & 11.02342 \\
\hline 14 & 36.9092 & 86.556 & 62 & 126 & 22.1036 & 6.855235 & 7.035794 & 6.945515 \\
\hline 15 & 32.2473 & 85.6288 & 56 & 123 & 21.5432 & 6.407694 & 6.857414 & 6.632554 \\
\hline 16 & 66.7747 & 89.3208 & 62 & 135 & 30.2875 & 9.220639 & 9.640811 & 9.430725 \\
\hline 17 & 66.7869 & 84.7482 & 59 & 123 & 29.8912 & 9.221482 & 9.514664 & 9.368073 \\
\hline 18 & 39.0959 & 85.7504 & 59 & 122 & 22.8958 & 7.055384 & 7.287959 & 7.171672 \\
\hline 19 & 86.8693 & 85.3448 & 56 & 143 & 35.4597 & 10.51691 & 11.28717 & 10.90204 \\
\hline 20 & 95.3437 & 87.6245 & 55 & 129 & 35.6277 & 11.01796 & 11.34065 & 11.1793 \\
\hline 21 & 69.376 & 86.968 & 51 & 125 & 30.1809 & 9.398525 & 9.606879 & 9.502702 \\
\hline 22 & 65.1205 & 89.6056 & 58 & 133 & 29.5944 & 9.105712 & 9.42019 & 9.262951 \\
\hline 23 & 45.5339 & 84.4758 & 60 & 120 & 24.5802 & 7.614169 & 7.824121 & 7.719145 \\
\hline 24 & 42.1117 & 93.8546 & 55 & 130 & 23.6087 & 7.322451 & 7.514883 & 7.418667 \\
\hline 25 & 84.6826 & 91.9836 & 56 & 127 & 35.2202 & 10.3837 & 11.21094 & 10.79732 \\
\hline 26 & 89.4787 & 85.1524 & 53 & 143 & 35.1643 & 10.6737 & 11.19314 & 10.93342 \\
\hline 27 & 131.1351 & 86.1811 & 55 & 133 & 41.9901 & 12.92155 & 13.36586 & 13.14371 \\
\hline 28 & 75.0133 & 90.7622 & 61 & 139 & 31.3901 & 9.772917 & 9.991779 & 9.882348 \\
\hline 29 & 87.5684 & 86.4981 & 63 & 129 & 35.8386 & 10.55915 & 11.40778 & 10.98346 \\
\hline 30 & 72.7413 & 87.5652 & 54 & 129 & 31.658 & 9.623778 & 10.07705 & 9.850416 \\
\hline 31 & 61.5641 & 86.4639 & 60 & 125 & 28.8804 & 8.853578 & 9.192917 & 9.02324 \\
\hline
\end{tabular}


Sample: 12.028.2.1

\begin{tabular}{rrrrrrrrr} 
& \multicolumn{1}{c}{ Area } & Mean & Min & Max & Perim. & Diam (using A) & Diam (using P) & Average D \\
1 & 9.1531 & 75.8606 & 52 & 95 & 11.2497 & 3.413809 & 3.580891 & 3.49735 \\
2 & 10.3521 & 74.3632 & 52 & 98 & 12.4643 & 3.630524 & 3.96751 & 3.799017 \\
3 & 12.8762 & 81.7639 & 53 & 106 & 14.1785 & 4.049011 & 4.513157 & 4.281084 \\
4 & 9.9579 & 72.9286 & 56 & 92 & 12.1602 & 3.560729 & 3.870712 & 3.715721 \\
5 & 10.7423 & 75.3299 & 52 & 95 & 12.3844 & 3.698313 & 3.942077 & 3.820195 \\
6 & 7.3079 & 74.2603 & 49 & 98 & 9.8995 & 3.050362 & 3.151109 & 3.100735 \\
7 & 8.3565 & 77.1342 & 57 & 95 & 10.7397 & 3.261875 & 3.418553 & 3.340214 \\
8 & 10.1896 & 67.8205 & 45 & 94 & 11.8096 & 3.601916 & 3.759112 & 3.680514 \\
9 & 9.4498 & 70.0662 & 50 & 90 & 11.5045 & 3.468697 & 3.661996 & 3.565347 \\
10 & 10.7748 & 60.4738 & 45 & 85 & 12.3441 & 3.703904 & 3.929249 & 3.816576 \\
11 & 9.5758 & 67.5497 & 50 & 98 & 11.4437 & 3.491746 & 3.642643 & 3.567194 \\
12 & 15.7538 & 53.5106 & 32 & 85 & 14.478 & 4.478656 & 4.608491 & 4.543573 \\
13 & 10.8155 & 68.6659 & 49 & 87 & 11.9653 & 3.710892 & 3.808673 & 3.759783 \\
14 & 9.7912 & 73.5509 & 52 & 91 & 11.7393 & 3.530799 & 3.736735 & 3.633767 \\
15 & 7.2875 & 72.4138 & 55 & 90 & 10.1376 & 3.046101 & 3.226898 & 3.1365 \\
16 & 8.7467 & 72.4266 & 58 & 86 & 11.1619 & 3.337161 & 3.552943 & 3.445052 \\
17 & 13.4777 & 72.7979 & 54 & 95 & 13.733 & 4.142504 & 4.37135 & 4.256927 \\
18 & 12.108 & 68.4371 & 54 & 80 & 12.6201 & 3.92637 & 4.017103 & 3.971736 \\
19 & 11.7422 & 62.3752 & 51 & 85 & 12.8058 & 3.866605 & 4.076213 & 3.971409 \\
20 & 10.9659 & 61.5226 & 49 & 80 & 12.2546 & 3.736605 & 3.90076 & 3.818683 \\
21 & 7.2632 & 64.1147 & 49 & 102 & 10.3211 & 3.041018 & 3.285308 & 3.163163 \\
22 & 8.0964 & 66.9563 & 48 & 86 & 10.6608 & 3.21071 & 3.393438 & 3.302074 \\
23 & 9.7912 & 57.9543 & 47 & 78 & 11.4111 & 3.530799 & 3.632266 & 3.581533 \\
24 & 10.157 & 57.3093 & 36 & 78 & 11.9309 & 3.59615 & 3.797723 & 3.696937 \\
25 & 12.3112 & 55.2595 & 42 & 73 & 12.9668 & 3.95918 & 4.127461 & 4.04332 \\
26 & 14.4613 & 37.0149 & 23 & 52 & 14.5856 & 4.291002 & 4.642741 & 4.466871 \\
27 & 8.9336 & 51.7152 & 32 & 68 & 11.2547 & 3.372627 & 3.582482 & 3.477555 \\
28 & 8.7711 & 47.336 & 35 & 62 & 11.2672 & 3.341813 & 3.586461 & 3.464137
\end{tabular}


Sample: 12.028.2.2

\begin{tabular}{rrrrrrrrr} 
& \multicolumn{1}{c}{ Area } & Mean & Min & Max & Perim. & Diam (using A) & Diam (using P) & Average D \\
1 & 15.1197 & 73.5774 & 56 & 100 & 14.4917 & 4.387596 & 4.612851 & 4.500224 \\
2 & 13.4127 & 77.8261 & 54 & 98 & 13.6337 & 4.132503 & 4.339741 & 4.236122 \\
3 & 10.5635 & 78.9681 & 63 & 98 & 12.3675 & 3.667406 & 3.936698 & 3.802052 \\
4 & 9.1287 & 68.89 & 47 & 96 & 11.2437 & 3.409255 & 3.578981 & 3.494118 \\
5 & 12.4413 & 72.3976 & 54 & 102 & 13.2001 & 3.980045 & 4.201722 & 4.090883 \\
6 & 9.3604 & 77.3752 & 63 & 95 & 11.2595 & 3.45225 & 3.58401 & 3.51813 \\
7 & 9.6937 & 79.0122 & 55 & 112 & 11.6299 & 3.513176 & 3.701912 & 3.607544 \\
8 & 11.3073 & 80.5482 & 59 & 112 & 12.303 & 3.794325 & 3.916167 & 3.855246 \\
9 & 10.279 & 81.9518 & 61 & 109 & 11.6733 & 3.617683 & 3.715727 & 3.666705 \\
10 & 10.9862 & 78.5797 & 57 & 100 & 12.6091 & 3.740062 & 4.013601 & 3.876832 \\
11 & 13.6321 & 55.2976 & 35 & 123 & 14.3143 & 4.166165 & 4.556383 & 4.361274 \\
12 & 13.185 & 70.7028 & 52 & 88 & 13.6493 & 4.097275 & 4.344707 & 4.220991 \\
13 & 15.957 & 69.8136 & 56 & 84 & 14.8239 & 4.507448 & 4.718594 & 4.613021 \\
14 & 12.3925 & 70.8285 & 48 & 89 & 13.3335 & 3.972231 & 4.244185 & 4.108208 \\
15 & 14.693 & 74.1071 & 50 & 98 & 15.3799 & 4.325241 & 4.895574 & 4.610408 \\
16 & 8.3849 & 79.5313 & 62 & 112 & 10.6917 & 3.267413 & 3.403274 & 3.335343 \\
17 & 11.3276 & 53.8159 & 35 & 104 & 12.4754 & 3.797729 & 3.971043 & 3.884386 \\
18 & 7.9907 & 71.6424 & 50 & 92 & 10.611 & 3.189683 & 3.377586 & 3.283634 \\
19 & 8.0313 & 71.6397 & 50 & 98 & 10.6967 & 3.197776 & 3.404865 & 3.30132 \\
20 & 6.1698 & 84.334 & 60 & 102 & 9.2294 & 2.80279 & 2.937809 & 2.8703 \\
21 & 4.288 & 80.5592 & 61 & 99 & 8.0499 & 2.33659 & 2.562363 & 2.449476 \\
22 & 13.185 & 75.9069 & 53 & 99 & 13.4501 & 4.097275 & 4.2813 & 4.189287 \\
23 & 11.5227 & 60.4469 & 36 & 139 & 12.4976 & 3.830295 & 3.97811 & 3.904202 \\
24 & 10.913 & 75.1918 & 51 & 101 & 12.3252 & 3.727581 & 3.923233 & 3.825407 \\
25 & 11.3195 & 57.47 & 30 & 111 & 12.4739 & 3.796371 & 3.970566 & 3.883468 \\
26 & 12.8518 & 70.3327 & 48 & 89 & 13.9253 & 4.045172 & 4.432561 & 4.238867
\end{tabular}


Sample: 12.028.3.1

\begin{tabular}{rrrrrrrrr} 
& \multicolumn{1}{l}{ Area } & \multicolumn{1}{c}{ Mean } & Min & Max & Perim. & Diam (using A) & Diam (using P) & Average D \\
1 & 78.8095 & 115.4305 & 77 & 163 & 33.1928 & 10.01715 & 10.5656 & 10.29138 \\
2 & 38.4618 & 115.0361 & 82 & 158 & 22.6314 & 6.997934 & 7.203798 & 7.100866 \\
3 & 45.0747 & 103.2017 & 70 & 139 & 25.8512 & 7.575678 & 8.228693 & 7.902185 \\
4 & 43.5668 & 104.1046 & 70 & 129 & 23.8972 & 7.447884 & 7.606715 & 7.527299 \\
5 & 70.7782 & 111.4082 & 78 & 152 & 31.9577 & 9.493029 & 10.17245 & 9.832741 \\
6 & 79.0859 & 110.7625 & 85 & 144 & 33.928 & 10.0347 & 10.79962 & 10.41716 \\
7 & 94.9372 & 102.2321 & 69 & 139 & 35.7057 & 10.99444 & 11.36548 & 11.17996 \\
8 & 132.6715 & 66.3828 & 39 & 120 & 42.5033 & 12.99702 & 13.52922 & 13.26312 \\
9 & 79.0859 & 109.4768 & 81 & 176 & 32.455 & 10.0347 & 10.33075 & 10.18273 \\
10 & 75.3263 & 115.4132 & 83 & 160 & 32.2356 & 9.793285 & 10.26091 & 10.0271 \\
11 & 90.2306 & 112.1494 & 72 & 155 & 34.5312 & 10.71845 & 10.99162 & 10.85504 \\
12 & 75.2328 & 103.2489 & 68 & 145 & 32.7053 & 9.787205 & 10.41042 & 10.09881 \\
13 & 71.9244 & 107.5847 & 73 & 143 & 30.9184 & 9.569587 & 9.841632 & 9.70561 \\
14 & 112.5972 & 115.6456 & 79 & 158 & 38.5555 & 11.97344 & 12.2726 & 12.12302 \\
15 & 105.7283 & 118.5683 & 78 & 171 & 39.3802 & 11.60248 & 12.53511 & 12.06879 \\
16 & 44.6276 & 96.3694 & 59 & 147 & 25.8693 & 7.538012 & 8.234454 & 7.886233 \\
17 & 82.4635 & 115.755 & 70 & 155 & 34.1534 & 10.24675 & 10.87136 & 10.55906 \\
18 & 81.1832 & 102.9145 & 68 & 145 & 33.0047 & 10.16689 & 10.50572 & 10.33631 \\
19 & 100.6356 & 111.3244 & 72 & 155 & 36.331 & 11.31959 & 11.56452 & 11.44206 \\
20 & 90.8078 & 98.4906 & 45 & 151 & 34.6377 & 10.75268 & 11.02552 & 10.8891 \\
21 & 72.8917 & 116.9795 & 78 & 162 & 30.9981 & 9.633722 & 9.867002 & 9.750362 \\
22 & 94.8234 & 115.0727 & 81 & 158 & 35.1277 & 10.98785 & 11.18149 & 11.08467 \\
23 & 33.8162 & 113.352 & 69 & 146 & 21.5168 & 6.561716 & 6.84901 & 6.705363 \\
24 & 55.4796 & 115.2544 & 83 & 147 & 27.4648 & 8.40469 & 8.742317 & 8.573504 \\
25 & 50.9762 & 116.1447 & 86 & 157 & 26.8069 & 8.056359 & 8.532901 & 8.29463 \\
26 & 68.5956 & 121.5901 & 84 & 153 & 30.5755 & 9.345514 & 9.732484 & 9.538999 \\
27 & 60.8366 & 128.3787 & 96 & 159 & 29.8039 & 8.801112 & 9.486876 & 9.143994 \\
28 & 62.9867 & 116.7661 & 86 & 157 & 29.1436 & 8.955287 & 9.276696 & 9.115991
\end{tabular}


Sample: 12.028.3.1

\begin{tabular}{rrrrrrrrr} 
& \multicolumn{1}{l}{ Area } & \multicolumn{1}{c}{ Mean } & Min & Max & Perim. & Diam (using A) & Diam (using P) & Average D \\
1 & 82.9837 & 119.1332 & 85 & 190 & 33.2651 & 10.27901 & 10.58861 & 10.43381 \\
2 & 80.5979 & 117.9704 & 86 & 158 & 32.3038 & 10.13017 & 10.28262 & 10.2064 \\
3 & 83.3454 & 114.3326 & 81 & 163 & 34.7892 & 10.30139 & 11.07375 & 10.68757 \\
4 & 59.7676 & 111.0705 & 81 & 142 & 28.3655 & 8.723444 & 9.029019 & 8.876231 \\
5 & 49.765 & 112.5706 & 85 & 148 & 26.6579 & 7.960073 & 8.485473 & 8.222773 \\
6 & 83.3089 & 116.4158 & 79 & 158 & 32.9622 & 10.29914 & 10.49219 & 10.39566 \\
7 & 47.8425 & 115.88 & 84 & 148 & 26.4088 & 7.804804 & 8.406182 & 8.105493 \\
8 & 67.2909 & 117.6669 & 89 & 155 & 30.1732 & 9.256211 & 9.604428 & 9.430319 \\
9 & 64.393 & 122.8082 & 79 & 152 & 29.2033 & 9.054707 & 9.295699 & 9.175203 \\
10 & 80.0045 & 121.0675 & 79 & 170 & 32.8741 & 10.09281 & 10.46415 & 10.27848 \\
11 & 73.0096 & 114.427 & 82 & 153 & 31.2584 & 9.64151 & 9.949858 & 9.795684 \\
12 & 63.072 & 118.6081 & 75 & 167 & 30.0942 & 8.961348 & 9.579281 & 9.270315 \\
13 & 52.6223 & 122.4705 & 83 & 157 & 27.4121 & 8.185401 & 8.725542 & 8.455472 \\
14 & 75.1718 & 122.7157 & 88 & 160 & 31.5978 & 9.783236 & 10.05789 & 9.920564 \\
15 & 60.231 & 119.441 & 79 & 152 & 29.7574 & 8.757197 & 9.472075 & 9.114636 \\
16 & 61.4991 & 120.8784 & 83 & 157 & 29.0751 & 8.848903 & 9.254892 & 9.051897 \\
17 & 58.8165 & 117.5038 & 83 & 153 & 28.7013 & 8.653756 & 9.135908 & 8.894832 \\
18 & 61.6332 & 124.3264 & 86 & 158 & 28.3229 & 8.858545 & 9.015459 & 8.937002 \\
19 & 62.7956 & 112.1819 & 82 & 147 & 28.6738 & 8.941691 & 9.127154 & 9.034423 \\
20 & 48.2043 & 124.4726 & 91 & 162 & 25.0028 & 7.834259 & 7.958638 & 7.896449 \\
21 & 57.1339 & 118.9985 & 87 & 162 & 27.4376 & 8.529076 & 8.733659 & 8.631368 \\
22 & 51.6103 & 114.8006 & 83 & 154 & 26.2104 & 8.106311 & 8.343029 & 8.22467 \\
23 & 48.1677 & 122.676 & 83 & 157 & 25.3311 & 7.831285 & 8.06314 & 7.947212 \\
24 & 92.5758 & 111.2137 & 84 & 151 & 34.9831 & 10.85685 & 11.13547 & 10.99616 \\
25 & 73.229 & 102.5956 & 69 & 136 & 32.4723 & 9.655986 & 10.33625 & 9.99612
\end{tabular}


Sample: 12.028.4.1

\begin{tabular}{rrrrrrrrr} 
& \multicolumn{1}{l}{ Area } & Mean & Min & Max & Perim. & Diam (using A) & Diam (using P) & Average D \\
1 & 83.8616 & 116.2334 & 80 & 154 & 33.7197 & 10.33324 & 10.73331 & 10.53328 \\
2 & 99.2984 & 121.3361 & 86 & 154 & 36.4578 & 11.24414 & 11.60488 & 11.42451 \\
3 & 78.5331 & 119.455 & 94 & 151 & 32.3218 & 9.999572 & 10.28835 & 10.14396 \\
4 & 94.7421 & 117.7187 & 83 & 150 & 35.5558 & 10.98314 & 11.31776 & 11.15045 \\
5 & 83.4714 & 120.3775 & 86 & 171 & 33.5885 & 10.30917 & 10.69155 & 10.50036 \\
6 & 56.5608 & 120.6223 & 83 & 158 & 27.835 & 8.486192 & 8.860156 & 8.673174 \\
7 & 71.1602 & 114.6326 & 80 & 157 & 30.3356 & 9.518612 & 9.656121 & 9.587367 \\
8 & 87.479 & 113.006 & 77 & 151 & 35.0945 & 10.55375 & 11.17093 & 10.86234 \\
9 & 60.6699 & 113.7706 & 84 & 149 & 28.7155 & 8.789045 & 9.140428 & 8.964736 \\
10 & 65.5107 & 110.4944 & 78 & 146 & 31.3907 & 9.132952 & 9.99197 & 9.562461 \\
11 & 67.1283 & 125.2678 & 82 & 163 & 30.2489 & 9.245021 & 9.628524 & 9.436772 \\
12 & 58.3207 & 121.2369 & 91 & 153 & 27.75 & 8.617205 & 8.833099 & 8.725152 \\
13 & 69.0833 & 115.5393 & 88 & 147 & 30.1948 & 9.378677 & 9.611303 & 9.49499 \\
14 & 67.2137 & 120.8933 & 91 & 157 & 29.5263 & 9.250899 & 9.398513 & 9.324706 \\
15 & 78.4193 & 114.4106 & 81 & 149 & 32.2365 & 9.992325 & 10.2612 & 10.12676 \\
16 & 110.7804 & 115.2216 & 82 & 170 & 38.8322 & 11.87645 & 12.36067 & 12.11856 \\
17 & 95.681 & 113.9726 & 74 & 160 & 35.8703 & 11.03743 & 11.41787 & 11.22765 \\
18 & 35.5192 & 107.3832 & 74 & 149 & 22.1414 & 6.724913 & 7.047827 & 6.88637 \\
19 & 74.485 & 116.7521 & 78 & 168 & 31.8 & 9.738442 & 10.12225 & 9.930348 \\
20 & 56.0121 & 114.0788 & 79 & 143 & 28.2664 & 8.444929 & 8.997475 & 8.721202 \\
21 & 80.6629 & 112.3432 & 77 & 155 & 33.5586 & 10.13426 & 10.68203 & 10.40815 \\
22 & 114.7433 & 123.3415 & 76 & 158 & 39.6285 & 12.08701 & 12.61414 & 12.35057 \\
23 & 49.1594 & 114.4448 & 78 & 147 & 26.8285 & 7.911491 & 8.539777 & 8.225634 \\
24 & 45.8388 & 116.8012 & 93 & 144 & 25.5751 & 7.639619 & 8.140807 & 7.890213 \\
25 & 60.2838 & 115.745 & 77 & 171 & 28.1757 & 8.761034 & 8.968604 & 8.864819 \\
26 & 54.3009 & 89.3909 & 49 & 131 & 27.3165 & 8.31493 & 8.695112 & 8.505021 \\
27 & 46.1964 & 118.3216 & 76 & 159 & 25.8109 & 7.66936 & 8.215865 & 7.942612
\end{tabular}


Sample: 12.028.5.1

\begin{tabular}{rrrrrrrrr} 
& \multicolumn{1}{c}{ Area } & Mean & Min & Max & Perim. & Diam (using A) & Diam (using P) & Average D \\
1 & 10.7301 & 166.4178 & 138 & 194 & 12.1041 & 3.696213 & 3.852855 & 3.774534 \\
2 & 6.4543 & 140.8659 & 105 & 160 & 9.5039 & 2.866683 & 3.025185 & 2.945934 \\
3 & 7.6046 & 166.6526 & 106 & 194 & 10.711 & 3.111668 & 3.409417 & 3.260543 \\
4 & 12.9615 & 136.4785 & 93 & 176 & 13.7336 & 4.0624 & 4.371541 & 4.21697 \\
5 & 7.95 & 162.9755 & 103 & 193 & 11.0806 & 3.181549 & 3.527065 & 3.354307 \\
6 & 10.157 & 140.0088 & 95 & 186 & 11.9902 & 3.59615 & 3.816599 & 3.706375 \\
7 & 12.8111 & 130.3912 & 86 & 174 & 13.1551 & 4.038762 & 4.187398 & 4.11308 \\
8 & 14.6401 & 147.2282 & 112 & 166 & 14.2951 & 4.317448 & 4.550272 & 4.43386 \\
9 & 9.4823 & 157.1569 & 125 & 185 & 11.8349 & 3.474657 & 3.767166 & 3.620911 \\
10 & 11.7178 & 142.2345 & 98 & 187 & 12.8029 & 3.862585 & 4.07529 & 3.968938 \\
11 & 7.4501 & 132.5963 & 81 & 179 & 10.0034 & 3.079896 & 3.184181 & 3.132039 \\
12 & 10.9699 & 135.2864 & 88 & 172 & 12.0278 & 3.737287 & 3.828568 & 3.782927 \\
13 & 7.9582 & 127.2513 & 64 & 192 & 10.5176 & 3.183189 & 3.347856 & 3.265523 \\
14 & 7.0681 & 126.13 & 70 & 160 & 9.8764 & 2.999897 & 3.143756 & 3.071827 \\
15 & 6.4015 & 124.1511 & 81 & 157 & 9.3807 & 2.854933 & 2.98597 & 2.920451 \\
16 & 7.125 & 98.4997 & 63 & 157 & 10.4284 & 3.011948 & 3.319463 & 3.165705 \\
17 & 12.8477 & 150.702 & 113 & 182 & 13.1254 & 4.044527 & 4.177945 & 4.111236 \\
18 & 9.458 & 140.8483 & 92 & 176 & 11.6671 & 3.470202 & 3.713753 & 3.591977 \\
19 & 10.0961 & 135.0612 & 76 & 175 & 12.3584 & 3.585353 & 3.933801 & 3.759577 \\
20 & 6.5031 & 105.8962 & 68 & 143 & 10.4971 & 2.8775 & 3.341331 & 3.109415 \\
21 & 8.9214 & 137 & 102 & 174 & 11.5062 & 3.370323 & 3.662537 & 3.51643 \\
22 & 3.5808 & 107.9092 & 69 & 156 & 8.2135 & 2.135232 & 2.614438 & 2.374835 \\
23 & 11.2707 & 121.9174 & 74 & 166 & 12.4144 & 3.788179 & 3.951626 & 3.869903 \\
24 & 7.6249 & 154.5949 & 107 & 178 & 10.4707 & 3.115818 & 3.332927 & 3.224373 \\
25 & 8.9296 & 160.1124 & 117 & 186 & 11.1139 & 3.371872 & 3.537664 & 3.454768 \\
26 & 6.6128 & 116.5605 & 80 & 156 & 10.0138 & 2.901668 & 3.187492 & 3.04458 \\
27 & 7.6005 & 136.6989 & 104 & 175 & 10.3692 & 3.110829 & 3.300619 & 3.205724 \\
28 & 15.7456 & 155.0534 & 118 & 195 & 15.2878 & 4.47749 & 4.866258 & 4.671874
\end{tabular}


Sample: 12.028.5.2

\begin{tabular}{rrrrrrrrr}
\multicolumn{1}{r}{ Area } & Mean & Min & Max & Perim. & Diam (using A) & Diam (using P) & Average D \\
1 & 73.2575 & 118.3002 & 72 & 191 & 30.8987 & 9.657864 & 9.835362 & 9.746613 \\
2 & 61.5925 & 122.9098 & 77 & 186 & 28.9411 & 8.85562 & 9.212238 & 9.033929 \\
3 & 40.3843 & 117.9231 & 83 & 173 & 23.9943 & 7.170696 & 7.637623 & 7.40416 \\
4 & 69.7905 & 120.7892 & 76 & 168 & 30.6248 & 9.42656 & 9.748177 & 9.587368 \\
5 & 72.3593 & 121.7994 & 82 & 172 & 30.6383 & 9.598475 & 9.752474 & 9.675474 \\
6 & 62.3932 & 122.5856 & 78 & 171 & 29.1292 & 8.912996 & 9.272112 & 9.092554 \\
7 & 85.9182 & 122.4486 & 74 & 175 & 34.8842 & 10.45918 & 11.10399 & 10.78158 \\
8 & 78.7404 & 121.4291 & 79 & 170 & 32.379 & 10.01276 & 10.30656 & 10.15966 \\
9 & 75.5498 & 121.7657 & 83 & 161 & 31.6776 & 9.807803 & 10.08329 & 9.945548 \\
10 & 70.9855 & 123.2955 & 77 & 186 & 31.0544 & 9.506921 & 9.884923 & 9.695922 \\
11 & 59.1458 & 116.4623 & 78 & 176 & 28.1821 & 8.677947 & 8.970641 & 8.824294 \\
12 & 50.0455 & 122.2662 & 81 & 179 & 26.1897 & 7.982475 & 8.33644 & 8.159458 \\
13 & 87.5074 & 125.957 & 82 & 177 & 34.4786 & 10.55547 & 10.97488 & 10.76517 \\
14 & 75.4848 & 123.3844 & 83 & 180 & 31.4624 & 9.803583 & 10.01479 & 9.909188 \\
15 & 55.1138 & 125.3476 & 87 & 166 & 27.0925 & 8.376937 & 8.623811 & 8.500374 \\
16 & 85.0078 & 128.9011 & 81 & 184 & 34.0847 & 10.40362 & 10.8495 & 10.62656 \\
17 & 101.0623 & 123.0127 & 83 & 167 & 37.9439 & 11.34357 & 12.07792 & 11.71074 \\
18 & 89.4746 & 131.6503 & 77 & 184 & 35.6292 & 10.67345 & 11.34113 & 11.00729 \\
19 & 82.4228 & 124.7247 & 82 & 170 & 33.9575 & 10.24422 & 10.80901 & 10.52661 \\
20 & 79.0615 & 120.6226 & 80 & 163 & 33.2376 & 10.03316 & 10.57986 & 10.30651 \\
21 & 61.4462 & 121.4785 & 78 & 164 & 28.2943 & 8.845096 & 9.006355 & 8.925726 \\
22 & 77.5374 & 129.8218 & 77 & 184 & 35.536 & 9.935979 & 11.31146 & 10.62372 \\
23 & 116.6373 & 135.5021 & 86 & 182 & 40.3713 & 12.18635 & 12.85058 & 12.51847 \\
24 & 59.3612 & 125.9423 & 83 & 167 & 28.5858 & 8.693735 & 9.099143 & 8.896439 \\
25 & 69.6564 & 134.6757 & 87 & 191 & 30.3849 & 9.417499 & 9.671814 & 9.544656 \\
26 & 81.74 & 127.3734 & 81 & 175 & 33.538 & 10.2017 & 10.67548 & 10.43859 \\
27 & 64.2629 & 114.4749 & 77 & 166 & 29.0143 & 9.045555 & 9.235539 & 9.140547
\end{tabular}


Sample: 12.029.1.1

$\begin{array}{rrrrrrrrr} & \text { Area } & \text { Mean } & \text { Min } & \text { Max } & \text { Perim. } & \text { Diam (using A) } & \text { Diam (using P) } & \text { Average D } \\ 1 & 74.8345 & 123.9549 & 92 & 163 & 32.5892 & 9.761262 & 10.37346 & 10.06736 \\ 2 & 87.9952 & 135.7201 & 92 & 175 & 34.3988 & 10.58485 & 10.94948 & 10.76716 \\ 3 & 94.2544 & 131.6187 & 87 & 184 & 36.9454 & 10.95484 & 11.76009 & 11.35746 \\ & 102.005 & & & & & & & \\ 4 & 3 & 129.1601 & 84 & 186 & 36.6626 & 11.39637 & 11.67007 & 11.53322 \\ 5 & 81.3092 & 132.1095 & 91 & 173 & 35.5777 & 10.17478 & 11.32473 & 10.74976 \\ 6 & 74.6475 & 131.8298 & 91 & 170 & 31.943 & 9.749059 & 10.16777 & 9.958416 \\ 7 & 38.2586 & 121.4103 & 84 & 154 & 22.8766 & 6.979424 & 7.281848 & 7.130636 \\ 8 & 92.397 & 124.9366 & 83 & 184 & 35.6083 & 10.84636 & 11.33447 & 11.09042 \\ 9 & 59.5359 & 101.7781 & 39 & 164 & 28.4943 & 8.706518 & 9.070017 & 8.888268 \\ 10 & 86.154 & 127.2589 & 93 & 170 & 35.2404 & 10.47352 & 11.21737 & 10.84545 \\ & 121.364 & & & & & & & \\ 11 & 2 & 93.1262 & 53 & 155 & 40.6514 & 12.43084 & 12.93974 & 12.68529 \\ 12 & 78.4071 & 127.3054 & 83 & 171 & 32.4195 & 9.991547 & 10.31945 & 10.1555 \\ & 101.720 & & & & & & & \\ 13 & 8 & 126.9202 & 82 & 159 & 36.3074 & 11.38046 & 11.557 & 11.46873 \\ 14 & 48.2571 & 125.4566 & 89 & 159 & 25.579 & 7.838549 & 8.142049 & 7.990299 \\ 15 & 74.3143 & 128.1488 & 81 & 183 & 31.6756 & 9.727276 & 10.08266 & 9.904966 \\ 16 & 68.0388 & 122.0328 & 82 & 162 & 30.2147 & 9.307507 & 9.617638 & 9.462572 \\ 17 & 99.4813 & 125.5475 & 87 & 172 & 37.2907 & 11.25449 & 11.87 & 11.56224 \\ 18 & 56.0934 & 122.3871 & 84 & 186 & 26.9554 & 8.451055 & 8.58017 & 8.515613 \\ 19 & 67.6567 & 112.6466 & 73 & 172 & 30.0162 & 9.281335 & 9.554453 & 9.417894 \\ 20 & 38.3643 & 110.1416 & 49 & 153 & 22.2733 & 6.989059 & 7.089812 & 7.039435 \\ 21 & 40.1038 & 116.4153 & 83 & 153 & 22.9872 & 7.14575 & 7.317053 & 7.231402 \\ 22 & 98.2335 & 121.6534 & 78 & 173 & 42.7112 & 11.18368 & 13.5954 & 12.38954 \\ 23 & 57.8411 & 113.3646 & 63 & 178 & 27.6743 & 8.5817 & 8.809003 & 8.695352 \\ 24 & 98.1807 & 129.1536 & 80 & 180 & 38.2244 & 11.18068 & 12.1672 & 11.67394 \\ 25 & 66.6812 & 131.1824 & 84 & 188 & 30.094 & 9.214182 & 9.579218 & 9.3967 \\ & & & & & & & & \end{array}$


Sample: 12.029.1.2

\begin{tabular}{|c|c|c|c|c|c|c|c|c|}
\hline & Area & Mean & Min & Max & Perim. & $\begin{array}{l}\text { Diam } \\
\text { (using A) }\end{array}$ & $\begin{array}{l}\text { Diam (using } \\
\text { P) }\end{array}$ & $\begin{array}{l}\text { Average } \\
\text { D }\end{array}$ \\
\hline 1 & 8.8076 & 112.2713 & 93 & 132 & 12.4462 & 3.348759 & 3.961749 & 3.655254 \\
\hline 2 & 8.8605 & 113.639 & 90 & 134 & 12.1998 & 3.3588 & 3.883317 & 3.621059 \\
\hline 3 & 11.7991 & 110.3317 & 84 & 137 & 13.4472 & 3.875962 & 4.280377 & 4.078169 \\
\hline 4 & 5.6252 & 112.0253 & 87 & 139 & 9.3141 & 2.676234 & 2.96477 & 2.820502 \\
\hline 5 & 9.4132 & 116.12 & 91 & 147 & 12.5026 & 3.461973 & 3.979701 & 3.720837 \\
\hline 6 & 8.3443 & 110.6873 & 85 & 133 & 11.664 & 3.259493 & 3.712767 & 3.48613 \\
\hline 7 & 10.4253 & 120.593 & 91 & 141 & 12.4423 & 3.643337 & 3.960507 & 3.801922 \\
\hline 8 & 10.661 & 111.7934 & 76 & 133 & 14.1366 & 3.684292 & 4.49982 & 4.092056 \\
\hline 9 & 4.7473 & 81.0933 & 55 & 137 & 9.2711 & 2.458546 & 2.951083 & 2.704815 \\
\hline 10 & 12.1608 & 110.7604 & 85 & 134 & 13.4011 & 3.934922 & 4.265703 & 4.100312 \\
\hline 11 & 16.5545 & 109.768 & 89 & 135 & 16.9793 & 4.591061 & 5.404679 & 4.99787 \\
\hline 12 & 12.4941 & 115.3243 & 93 & 146 & 13.2075 & 3.988481 & 4.204078 & 4.09628 \\
\hline 13 & 9.4092 & 105.4799 & 87 & 132 & 12.0845 & 3.461238 & 3.846616 & 3.653927 \\
\hline 14 & 15.0953 & 110.2092 & 73 & 145 & 15.7291 & 4.38 & 6728 & 4.695391 \\
\hline 15 & 10.0107 & 72.5489 & 43 & 124 & 12.3494 & 3.570157 & 3.930936 & 3.750546 \\
\hline 16 & 13.7012 & 107.8529 & 76 & 152 & 14.475 & 4.17671 & 4.607536 & 4.392123 \\
\hline 17 & 16.7049 & 108.7311 & 81 & 132 & 14.9493 & 4.611869 & 4.75851 & 4.68519 \\
\hline 18 & 8.4012 & 104.3638 & 69 & 137 & 11.6491 & 3.270587 & 3.708024 & 3.489305 \\
\hline 19 & 17.0178 & 103.2837 & 4 & 134 & 15.7037 & 62 & 8643 & 4.826752 \\
\hline 20 & 6.9258 & 108.0839 & 79 & 154 & 9.9594 & 2.969546 & 3.170175 & 3.069861 \\
\hline 21 & 9.1328 & 102.4424 & 79 & 128 & 11.5795 & 3.410021 & 3.685869 & 3.547945 \\
\hline 22 & 9.8278 & 105.0054 & 79 & 136 & 11.9646 & 3.537392 & 3.80845 & 3.672921 \\
\hline 23 & 8.8564 & 105.4016 & 77 & 148 & 13.1326 & 3.358023 & 4.180236 & 3.76913 \\
\hline 24 & 7.442 & 107.4014 & 91 & 131 & 10.9515 & 3.078222 & 3.485971 & 3.282096 \\
\hline 25 & 11.9617 & 96.6174 & 74 & 119 & 13.433 & 3.902577 & 4.275857 & 4.089217 \\
\hline 26 & 10.9903 & 111.453 & 80 & 149 & 12.4679 & 3.74076 & 3.968656 & 3.854708 \\
\hline 27 & 16.8512 & 117.4086 & 67 & 148 & 15.5755 & 4.632021 & 4.957836 & 4.794928 \\
\hline 28 & 6.7348 & 103.2227 & 76 & 121 & 9.7507 & 2.928312 & 3.103744 & 3.016028 \\
\hline 29 & 7.0112 & 99.2151 & 80 & 127 & 9.9929 & 2.987798 & 3.180839 & 3.084318 \\
\hline 30 & 6.938 & 106.3357 & 76 & 131 & 9.6927 & 2.97216 & 3.085282 & 3.028721 \\
\hline 31 & 5.2797 & 108.6266 & 79 & 131 & 8.3428 & 2.592744 & 2.655596 & 2.62417 \\
\hline 32 & 18.6436 & 103.5335 & 68 & 144 & 17.152 & 4.872142 & 5.459651 & 5.165897 \\
\hline 33 & 11.2504 & 99.4057 & 71 & 138 & 12.5906 & 3.784766 & 4.007712 & 3.896239 \\
\hline 34 & 9.3076 & 103.9921 & 74 & 136 & 11.5204 & 3.4425 & 3.667057 & 3.554778 \\
\hline 35 & 8.9743 & 108.1159 & 79 & 138 & 13.5181 & 3.380301 & 4.302945 & 3.841623 \\
\hline 36 & 8.4784 & 99.4492 & 73 & 124 & 11.3006 & 3.28558 & 3.597093 & 3.441336 \\
\hline 37 & 13.1972 & 112.8164 & 79 & 158 & 13.931 & 4.09917 & 4.434375 & 4.266773 \\
\hline 38 & 17.8388 & 78.5234 & 30 & 147 & 15.267 & 4.765823 & 4.859637 & 4.81273 \\
\hline 39 & 17.4934 & 104.826 & 78 & 135 & 17.498 & 4.719459 & 5.569786 & 5.144622 \\
\hline
\end{tabular}




$\begin{array}{rrrrrrrrr}40 & 7.3729 & 101.3037 & 72 & 130 & 11.4113 & 3.063897 & 3.63233 & 3.348114 \\ 41 & 14.1036 & 104.0977 & 72 & 141 & 14.1659 & 4.237601 & 4.509146 & 4.373373 \\ 42 & 15.6115 & 103.2317 & 78 & 129 & 14.6936 & 4.458383 & 4.677118 & 4.567751 \\ 43 & 10.5391 & 104.307 & 70 & 136 & 12.0011 & 3.663168 & 3.820069 & 3.741618 \\ 44 & 9.1775 & 101.7648 & 67 & 139 & 11.6497 & 3.418356 & 3.708215 & 3.563285 \\ 45 & 10.4741 & 95.4692 & 63 & 125 & 13.1662 & 3.651854 & 4.190932 & 3.921393 \\ 46 & 9.6693 & 111.467 & 77 & 135 & 13.6705 & 3.508751 & 4.351455 & 3.930103 \\ 47 & 9.9904 & 107.7327 & 76 & 134 & 11.5427 & 3.566535 & 3.674156 & 3.620345 \\ 48 & 16.0464 & 95.7343 & 68 & 129 & 15.0022 & 4.520057 & 4.775349 & 4.647703 \\ 49 & 9.9904 & 112.9402 & 93 & 144 & 12.5931 & 3.566535 & 4.008508 & 3.787522 \\ 50 & 13.685 & 116.4758 & 94 & 140 & 13.4104 & 4.17424 & 4.268663 & 4.221452 \\ 51 & 16.9934 & 104.6826 & 77 & 140 & 15.3734 & 4.651523 & 4.893505 & 4.772514 \\ 52 & 12.4494 & 113.4851 & 91 & 133 & 14.2887 & 3.98134 & 4.548234 & 4.264787 \\ 53 & 7.4135 & 113.79 & 88 & 131 & 11.3794 & 3.072322 & 3.622176 & 3.347249 \\ 54 & 8.4947 & 111.9344 & 90 & 138 & 10.8884 & 3.288737 & 3.465885 & 3.377311 \\ 55 & 8.8402 & 96.2423 & 71 & 123 & 15.0734 & 3.35495 & 4.798012 & 4.076481 \\ 56 & 10.6854 & 104.9 & 74 & 132 & 11.8719 & 3.688506 & 3.778943 & 3.733724 \\ 57 & 14.1361 & 103.891 & 78 & 138 & 13.6136 & 4.242481 & 4.333343 & 4.287912 \\ 58 & 12.9737 & 99.49 & 75 & 135 & 13.3354 & 4.064311 & 4.24479 & 4.154551 \\ 59 & 13.7094 & 104.2656 & 70 & 144 & 14.7086 & 4.17796 & 4.681893 & 4.429926 \\ 60 & 9.5474 & 108.1652 & 68 & 134 & 11.2664 & 3.486564 & 3.586207 & 3.536385 \\ 61 & 13.1647 & 94.7326 & 63 & 127 & 14.0175 & 4.09412 & 4.461909 & 4.278014 \\ 62 & 18.0786 & 96.2394 & 66 & 133 & 15.9784 & 4.797748 & 5.086083 & 4.941915\end{array}$


Sample: 12.029.2.1

$\begin{array}{rrrrrrrrr} & \text { Area } & \text { Mean } & \text { Min } & \text { Max } & \text { Perim. } & \text { Diam (using A) } & \text { Diam (using P) } & \text { Average D } \\ 1 & 46.7451 & 78.3232 & 53 & 114 & 25.2988 & 7.714772 & 8.052858 & 7.883815 \\ 2 & 59.4425 & 78.6461 & 55 & 119 & 27.8798 & 8.699686 & 8.874416 & 8.787051 \\ 3 & 28.5446 & 73.7522 & 52 & 104 & 19.7455 & 6.028608 & 6.285188 & 6.156898 \\ 4 & 30.5199 & 74.3376 & 57 & 119 & 20.4706 & 6.23371 & 6.515994 & 6.374852 \\ 5 & 60.3001 & 76.1329 & 49 & 111 & 28.8598 & 8.762218 & 9.18636 & 8.974289 \\ 6 & 39.8722 & 74.2337 & 50 & 117 & 24.6073 & 7.125087 & 7.832747 & 7.478917 \\ 7 & 54.2156 & 82.0763 & 56 & 113 & 27.3591 & 8.308396 & 8.708672 & 8.508534 \\ 8 & 53.1954 & 78.5063 & 52 & 114 & 27.2195 & 8.229853 & 8.664236 & 8.447045 \\ 9 & 41.7256 & 84.1295 & 61 & 114 & 23.8535 & 7.288805 & 7.592805 & 7.440805 \\ 10 & 22.5414 & 81.1774 & 54 & 114 & 18.6789 & 5.357294 & 5.945679 & 5.651486 \\ 11 & 28.4999 & 75.924 & 53 & 113 & 19.3739 & 6.023886 & 6.166904 & 6.095395 \\ 12 & 15.1726 & 65.45 & 49 & 90 & 15.0703 & 4.395265 & 4.797025 & 4.596145 \\ 13 & 24.976 & 76.5177 & 56 & 113 & 18.6481 & 5.639187 & 5.935875 & 5.787531 \\ 14 & 30.6093 & 72.6375 & 50 & 110 & 20.1901 & 6.242834 & 6.426708 & 6.334771 \\ 15 & 40.6078 & 77.4868 & 51 & 121 & 23.5744 & 7.190512 & 7.503965 & 7.347238 \\ 16 & 25.4759 & 76.5841 & 58 & 105 & 18.2481 & 5.695342 & 5.808551 & 5.751946 \\ 17 & 41.3923 & 73.9509 & 49 & 107 & 24.3275 & 7.259636 & 7.743684 & 7.50166 \\ 18 & 30.2516 & 74.7435 & 48 & 108 & 20.0442 & 6.20625 & 6.380267 & 6.293258 \\ 19 & 33.43 & 58.4469 & 29 & 83 & 21.8833 & 6.52414 & 6.965671 & 6.744905 \\ 20 & 39.0227 & 73.7333 & 54 & 101 & 22.9222 & 7.048776 & 7.296363 & 7.172569 \\ 21 & 47.3142 & 80.9444 & 59 & 115 & 25.258 & 7.761592 & 8.039871 & 7.900732 \\ 22 & 39.7665 & 72.1856 & 55 & 109 & 23.1674 & 7.115636 & 7.374412 & 7.245024 \\ 23 & 37.0596 & 73.9891 & 49 & 106 & 22.3022 & 6.869188 & 7.099011 & 6.9841 \\ 24 & 31.6783 & 71.652 & 53 & 112 & 20.4261 & 6.350911 & 6.50183 & 6.42637 \\ 25 & 58.9872 & 75.6499 & 52 & 112 & 28.7277 & 8.666305 & 9.144311 & 8.905308 \\ 26 & 40.616 & 69.1199 & 42 & 115 & 23.4294 & 7.191238 & 7.45781 & 7.324524 \\ 27 & 56.955 & 79.2478 & 55 & 121 & 29.7103 & 8.515712 & 9.457082 & 8.986397 \\ 28 & 45.9851 & 73.3946 & 53 & 105 & 24.8605 & 7.6518 & 7.913343 & 7.782572 \\ 29 & 36.2955 & 77.8191 & 56 & 116 & 22.8054 & 6.798005 & 7.259184 & 7.028594\end{array}$


Sample: 12.029.4.1

\begin{tabular}{rrrrrrrrr} 
& \multicolumn{1}{c}{ Area } & Mean & Min & Max & Perim. & Diam (using A) & Diam (using P) & Average D \\
1 & 10.4334 & 79.2855 & 48 & 118 & 11.8073 & 3.644752 & 3.75838 & 3.701566 \\
2 & 9.8441 & 81.5764 & 56 & 120 & 11.9562 & 3.540324 & 3.805777 & 3.673051 \\
3 & 12.5225 & 79.4619 & 53 & 110 & 13.2176 & 3.993012 & 4.207293 & 4.100152 \\
4 & 10.1611 & 53.5528 & 38 & 77 & 13.9606 & 3.596876 & 4.443797 & 4.020336 \\
5 & 3.9425 & 74.8763 & 55 & 127 & 7.6675 & 2.240479 & 2.440641 & 2.34056 \\
6 & 6.5478 & 85.0441 & 62 & 111 & 9.7911 & 2.887372 & 3.116604 & 3.001988 \\
7 & 6.4909 & 78.4652 & 48 & 122 & 9.5255 & 2.874799 & 3.032061 & 2.95343 \\
8 & 11.1 & 82.9348 & 58 & 122 & 12.7516 & 3.759383 & 4.05896 & 3.909172 \\
9 & 8.8036 & 78.4945 & 53 & 112 & 11.0539 & 3.347998 & 3.518566 & 3.433282 \\
10 & 14.7336 & 78.3743 & 48 & 126 & 14.3505 & 4.331213 & 4.567906 & 4.449559 \\
11 & 5.4301 & 79.1243 & 64 & 134 & 8.9201 & 2.629414 & 2.839356 & 2.734385 \\
12 & 13.7216 & 72.7722 & 49 & 121 & 14.6613 & 4.179819 & 4.666837 & 4.423328 \\
13 & 11.7218 & 72.4934 & 50 & 99 & 12.5996 & 3.863245 & 4.010577 & 3.936911 \\
14 & 20.0905 & 73.2082 & 44 & 121 & 17.1936 & 5.057669 & 5.472893 & 5.265281 \\
15 & 13.0834 & 72.972 & 45 & 105 & 13.8512 & 4.081458 & 4.408974 & 4.245216 \\
16 & 14.2337 & 78.3946 & 54 & 118 & 13.637 & 4.257101 & 4.340792 & 4.298947 \\
17 & 6.434 & 89.9128 & 64 & 124 & 9.6202 & 2.862171 & 3.062205 & 2.962188
\end{tabular}


Sample: 12.029.5.1

\begin{tabular}{|c|c|c|c|c|c|c|c|c|}
\hline & Area & Mean & Min & Max & Perim. & Diam (using A) & Diam (using P) & Average D \\
\hline 1 & 103.7327 & 104.9004 & 43 & 152 & 37.3447 & 11.49246 & 11.88719 & 11.68982 \\
\hline 2 & 49.9926 & 114.1567 & 77 & 173 & 25.7792 & 7.978255 & 8.205774 & 8.092015 \\
\hline 3 & 32.1579 & 109.6695 & 75 & 151 & 21.1737 & 6.398805 & 6.739798 & 6.569302 \\
\hline 4 & 26.8863 & 109.8907 & 76 & 162 & 19.0831 & 5.850872 & 6.074339 & 5.962606 \\
\hline 5 & 35.6492 & 111.9372 & 77 & 153 & 22.2538 & 6.737208 & 7.083605 & 6.910406 \\
\hline 6 & 45.0096 & 120.8884 & 85 & 157 & 24.8177 & 7.570205 & 7.899719 & 7.734962 \\
\hline 7 & 34.8973 & 114.9563 & 83 & 156 & 23.0573 & 6.66578 & 7.339367 & 7.002573 \\
\hline 8 & 30.6378 & 108.1434 & 78 & 144 & 20.4371 & 6.245739 & 6.505331 & 6.375535 \\
\hline 9 & 36.4255 & 109.293 & 69 & 155 & 22.5783 & 6.810168 & 7.186896 & 6.998532 \\
\hline 10 & 28.3251 & 113.445 & 74 & 151 & 19.1741 & 6.005384 & 6.103306 & 6.054345 \\
\hline 11 & 69.7458 & 117.2336 & 79 & 161 & 31.0221 & 9.42354 & 9.874641 & 9.649091 \\
\hline 12 & 31.0849 & 112.6437 & 75 & 154 & 24.5035 & 6.291146 & 7.799706 & 7.045426 \\
\hline 13 & 26.8944 & 110.1978 & 75 & 184 & 19.3325 & 5.851753 & 6.153726 & 6.002739 \\
\hline 14 & 38.2301 & 110.297 & 77 & 163 & 22.3721 & 6.976824 & 7.121261 & 7.049042 \\
\hline 15 & 46.7655 & 109.2769 & 70 & 155 & 27.7031 & 7.716455 & 8.818171 & 8.267313 \\
\hline 16 & 62.5314 & 108.9654 & 74 & 151 & 28.8292 & 8.922861 & 9.176619 & 9.04974 \\
\hline 17 & 97.7011 & 112.5485 & 79 & 161 & 36.0082 & 11.15334 & 11.46177 & 11.30755 \\
\hline 18 & 37.3482 & 124.4405 & 74 & 162 & 23.2696 & 6.895883 & 7.406944 & 7.151413 \\
\hline 19 & 46.1843 & 117.5217 & 78 & 168 & 24.8188 & 7.668356 & 7.900069 & 7.784212 \\
\hline 20 & 24.8825 & 126.9992 & 82 & 170 & 19.0119 & 5.628622 & 6.051676 & 5.840149 \\
\hline 21 & 32.2066 & 113.9107 & 80 & 148 & 21.3014 & 6.403649 & 6.780446 & 6.592047 \\
\hline 22 & 30.8898 & 115.2355 & 74 & 162 & 20.9787 & 6.271373 & 6.677728 & 6.47455 \\
\hline 23 & 26.6587 & 108.0666 & 76 & 153 & 19.8133 & 5.826055 & 6.306769 & 6.066412 \\
\hline 24 & 21.1432 & 106.0233 & 77 & 149 & 17.1788 & 5.188483 & 5.468182 & 5.328333 \\
\hline 25 & 29.52 & 107.2572 & 69 & 145 & 20.4294 & 6.130745 & 6.50288 & 6.316812 \\
\hline 26 & 55.7154 & 111.9217 & 74 & 154 & 28.0235 & 8.422532 & 8.920157 & 8.671345 \\
\hline 27 & 41.2785 & 120.9618 & 74 & 162 & 23.6054 & 7.24965 & 7.513832 & 7.381741 \\
\hline 28 & 45.8754 & 115.1281 & 78 & 168 & 25.6215 & 7.642668 & 8.155577 & 7.899122 \\
\hline 29 & 53.6384 & 106.2564 & 64 & 156 & 26.9935 & 8.264051 & 8.592298 & 8.428174 \\
\hline 30 & 23.6347 & 114.5634 & 77 & 149 & 19.57 & 5.485675 & 6.229324 & 5.8575 \\
\hline 31 & 54.5814 & 114.7954 & 75 & 156 & 26.6036 & 8.336378 & 8.468189 & 8.402283 \\
\hline 32 & 52.9922 & 118.9933 & 81 & 159 & 28.8715 & 8.21412 & 9.190084 & 8.702102 \\
\hline 33 & 24.346 & 111.7511 & 80 & 149 & 22.8393 & 5.567611 & 7.269975 & 6.418793 \\
\hline 34 & 50.208 & 100.633 & 64 & 146 & 25.7225 & 7.995424 & 8.187726 & 8.091575 \\
\hline 35 & 50.7486 & 121.6945 & 83 & 156 & 26.0028 & 8.038353 & 8.276948 & 8.157651 \\
\hline 36 & 45.9038 & 119.2133 & 82 & 155 & 26.0874 & 7.645033 & 8.303877 & 7.974455 \\
\hline 37 & 54.7318 & 111.2249 & 74 & 148 & 29.0044 & 8.347856 & 9.232387 & 8.790121 \\
\hline 38 & 53.0775 & 104.1626 & 70 & 138 & 27.6626 & 8.220728 & 8.805279 & 8.513004 \\
\hline 39 & 44.8105 & 110.979 & 72 & 151 & 25.6467 & 7.553443 & 8.163598 & 7.858521 \\
\hline 40 & 48.2327 & 119.4264 & 81 & 160 & 27.2697 & 7.836567 & 8.680215 & 8.258391 \\
\hline
\end{tabular}




$\begin{array}{lrrrrrrrr}41 & 39.5714 & 116.9815 & 75 & 150 & 26.2413 & 7.09816 & 8.352865 & 7.725512 \\ 42 & 27.5203 & 114.5497 & 78 & 157 & 19.8525 & 5.919454 & 6.319247 & 6.11935 \\ 43 & 33.6698 & 113.4965 & 74 & 160 & 21.7578 & 6.547497 & 6.925723 & 6.73661 \\ 44 & 53.988 & 119.3007 & 81 & 159 & 26.6017 & 8.290938 & 8.467584 & 8.379261 \\ 45 & 54.3741 & 115.429 & 55 & 166 & 28.5704 & 8.320532 & 9.094241 & 8.707386 \\ 46 & 36.08 & 118.5874 & 84 & 163 & 23.2204 & 6.777793 & 7.391283 & 7.084538 \\ 47 & 14.441 & 107.7166 & 71 & 145 & 15.1831 & 4.287989 & 4.832931 & 4.56046 \\ 48 & 39.9738 & 103.237 & 77 & 139 & 23.1234 & 7.134159 & 7.360407 & 7.247283 \\ 49 & 27.9877 & 100.3603 & 71 & 149 & 19.6019 & 5.96951 & 6.239479 & 6.104494\end{array}$


Sample: 12.029.5.2

\begin{tabular}{|c|c|c|c|c|c|c|c|c|}
\hline & Area & Mean & Min & Max & Perim. & Diam (using A) & Diam (using P) & Average $D$ \\
\hline 1 & 38.7504 & 118.1021 & 77 & 164 & 23.3487 & 7.02414 & 7.432122 & 7.228131 \\
\hline 2 & 33.0236 & 115.3534 & 84 & 160 & 21.7263 & 6.484362 & 6.915696 & 6.700029 \\
\hline 3 & 43.9285 & 115.4209 & 78 & 162 & 24.6024 & 7.478737 & 7.831187 & 7.654962 \\
\hline 4 & 39.9819 & 113.4704 & 81 & 167 & 23.0577 & 7.134882 & 7.339494 & 237188 \\
\hline 5 & 36.2548 & 111.8413 & 76 & 187 & 22.0251 & 6.794192 & 7.010807 & 6.9025 \\
\hline 6 & 45.1885 & 115.2806 & 77 & 161 & 25.1942 & 7.585235 & 8.019563 & 7.802399 \\
\hline 7 & 37.3034 & 108.719 & 75 & 147 & 22.3618 & 6.891746 & 7.117982 & 7.004864 \\
\hline 8 & 22.0496 & 113.9935 & 76 & 146 & 17.6804 & 5.29853 & 5.627846 & 5.463188 \\
\hline 9 & 29.2071 & 114.6618 & 75 & 161 & 21.8433 & 6.098167 & 6.952938 & 6.525552 \\
\hline 10 & 34.0031 & 114.6749 & 77 & 184 & 23.7404 & 6.579825 & 7.556804 & 7.068314 \\
\hline 11 & 39.4454 & 115.3813 & 70 & 156 & 23.2255 & 7.08685 & 7.392906 & 7.239878 \\
\hline 12 & 32.1822 & 110.4684 & 79 & 171 & 21.1279 & 6.401223 & 6.725219 & 6.563221 \\
\hline 13 & 31.8734 & 113.1368 & 71 & 174 & 20.7674 & 6.370437 & 6.610469 & 6.490453 \\
\hline 14 & 43.9488 & 120.2915 & 75 & 166 & 24.5195 & 7.480465 & 7.804799 & 2632 \\
\hline 15 & 29.6542 & 111.9729 & 74 & 154 & 19.633 & 6.144664 & 6.249378 & 6.197021 \\
\hline 16 & 34.0844 & 119.9174 & 87 & 152 & 21.6066 & 6.587686 & 6.877594 & 6.73264 \\
\hline 17 & 30.6825 & 119.3171 & 79 & 173 & 20.3406 & 6.250294 & 6.474614 & 6.362454 \\
\hline 18 & 97.0142 & 120.4398 & 75 & 171 & 37.0482 & 11.11406 & 11.79281 & 11.45343 \\
\hline 19 & 31.8083 & 115.5985 & 86 & 164 & 21.6158 & 6.363928 & 523 & 2226 \\
\hline 20 & 29.5282 & 105.9597 & 74 & 166 & 20.0822 & 6.131596 & 6.392363 & 6.261979 \\
\hline 21 & 41.819 & 121.4377 & 77 & 169 & 24.1327 & 7.296959 & 7.681677 & 7.489318 \\
\hline 24 & 39.2625 & 115.3763 & 79 & 149 & 23.3813 & 7.070401 & 7.442499 & 7.25645 \\
\hline 23 & 27.8292 & 118.8329 & 75 & 168 & 19.1679 & 5.952582 & 6.101332 & 6.026957 \\
\hline 24 & 29.0486 & 107.9723 & 70 & 156 & 19.9438 & 6.081597 & 6.348309 & 6.214953 \\
\hline 25 & 27.0895 & 111.6272 & 77 & 156 & 20.0647 & 5.87294 & 6.386792 & 6.129866 \\
\hline 26 & 32.796 & 106.4655 & 78 & 144 & 20.926 & 6.461978 & 6.660953 & 6.561466 \\
\hline 27 & 70.0466 & 110.6104 & 74 & 165 & 31.8554 & 9.443839 & .13989 & 9.791864 \\
\hline 28 & 57.0688 & 114.0022 & 74 & 166 & 28.1336 & 8.524216 & 8.955203 & 8.739709 \\
\hline 29 & 42.5059 & 126.0273 & 70 & 178 & 23.4762 & 7.356643 & 7.472707 & 7.414675 \\
\hline 30 & 34.2389 & 113.1668 & 75 & 154 & 22.2183 & 6.6026 & 7.072305 & 6.837452 \\
\hline 31 & 65.3115 & 117.6714 & 75 & 187 & 30.478 & 9.119056 & 9.701449 & 9.410252 \\
\hline ?ח & 33.2106 & 111.9217 & 77 & 156 & 21.3638 & 6.502696 & 6.800309 & 6.651502 \\
\hline 33 & 30.4752 & 93.6727 & 55 & 137 & 20.0183 & 6.229144 & 6.372023 & 6.300583 \\
\hline 34 & 43.9935 & 120.361 & 78 & 173 & 24.196 & 7.484268 & 7.701826 & 7.593047 \\
\hline 35 & 26.7156 & 124.6413 & 79 & 156 & 19.2972 & 5.832269 & 6.14249 & 5.987379 \\
\hline 36 & 29.1624 & 117.1236 & 83 & 155 & 19.7754 & 6.093498 & 6.294705 & 6.194102 \\
\hline 37 & 47.7613 & 111.0201 & 74 & 167 & 26.4454 & 7.798178 & 8.417832 & 8.108005 \\
\hline 38 & 33.2675 & 125.3146 & 78 & 173 & 22.6745 & 6.508264 & 7.217518 & 6.862891 \\
\hline 39 & 59.5766 & 122.0439 & 80 & 162 & 28.9512 & 8.709494 & 9.215453 & 8.962474 \\
\hline 40 & 40.1282 & 118.9754 & 73 & 158 & 23.8587 & 7.147924 & 7.59446 & 7.371192 \\
\hline
\end{tabular}




$\begin{array}{rrrrrrrrr}41 & 68.7622 & 114.5843 & 74 & 164 & 30.4954 & 9.356856 & 9.706987 & 9.531922 \\ 42 & 45.916 & 113.2266 & 70 & 151 & 25.104 & 7.646049 & 7.990851 & 7.81845 \\ 43 & 40.0226 & 112.3921 & 80 & 151 & 24.4092 & 7.138512 & 7.76969 & 7.454101 \\ 44 & 31.4466 & 116.6565 & 76 & 165 & 20.9581 & 6.327642 & 6.67117 & 6.499406 \\ 45 & 20.054 & 118.3468 & 81 & 158 & 16.5035 & 5.053073 & 5.253227 & 5.15315 \\ 46 & 38.161 & 120.8741 & 82 & 156 & 22.4418 & 6.970516 & 7.143447 & 7.056981 \\ 47 & 48.5741 & 121.8033 & 78 & 173 & 25.0368 & 7.864252 & 7.969461 & 7.916857 \\ 48 & 33.6007 & 115.9354 & 79 & 163 & 21.8432 & 6.540775 & 6.952907 & 6.746841 \\ 49 & 52.9272 & 122.872 & 80 & 162 & 28.3266 & 8.209081 & 9.016637 & 8.612859\end{array}$




\section{Block Information}

\#\#\#:

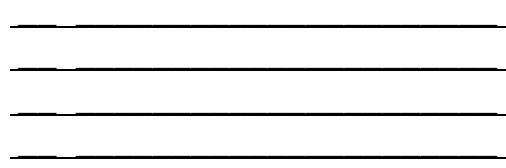

\section{Materials}

Section

1. Forceps (2)

2. Brushes (2)

3. Water bath (1)

4. Microtome (1)

5. Blade (1)

6. Cassette (1)

7. Slides (?)

Staining

8. xylene (3)

9. $95 \%(2)$

$10.100 \%(2)$

$11 . \mathrm{H}_{2} \mathrm{O}(2)$

12.Hematoxylin (1)

13.Bluing (1)

14.Clarifier (1)

15.Eosin (1)

16.Slide basket (1)

17.Cover slips (?)

18.Hemostats (1)

19.Glue

\section{Description-Section}

Prepare tissue for later analysis. Turn on water bath, set-up microtome, and position blade and cassette. Get sections on glass slips, label, and let air dry.

\section{Protocol-Staining}

20.Heat slides in oven till clear

21.3 min xylene $3 \mathrm{x}$

$22.2 \min 100 \% 2 \mathrm{x}$

$23.2 \min 95 \%$

24.1 min $\mathrm{H}_{2} \mathrm{O}$

25.4 min Hematoxylin

26.1 min $\mathrm{H}_{2} \mathrm{O}$
27.30 sec Clarifier

$28.1 \mathrm{~min}_{2} \mathrm{O}$

29.1 min Bluing

$30.1 \mathrm{~min}_{2} \mathrm{O}$

$31.1 \mathrm{~min} 95 \%$

$32.1 \mathrm{~min} 30 \mathrm{sec}$ Eosin

33.1 min $100 \% 3 x$

34.3 min xylene $3 x$

\section{Diagram}

\begin{tabular}{|c|c|c|c|}
\hline xylene & $95 \%$ & $\mathrm{H}_{2} \mathrm{O}$ & $95 \%$ \\
\hline xylene & $100 \%$ & Hematoxylin & Bluing \\
\hline xylene & $100 \%$ & $\mathrm{H}_{2} \mathrm{O}$ & Clarifier \\
\hline & Eosin & & \\
\hline
\end{tabular}

Notes

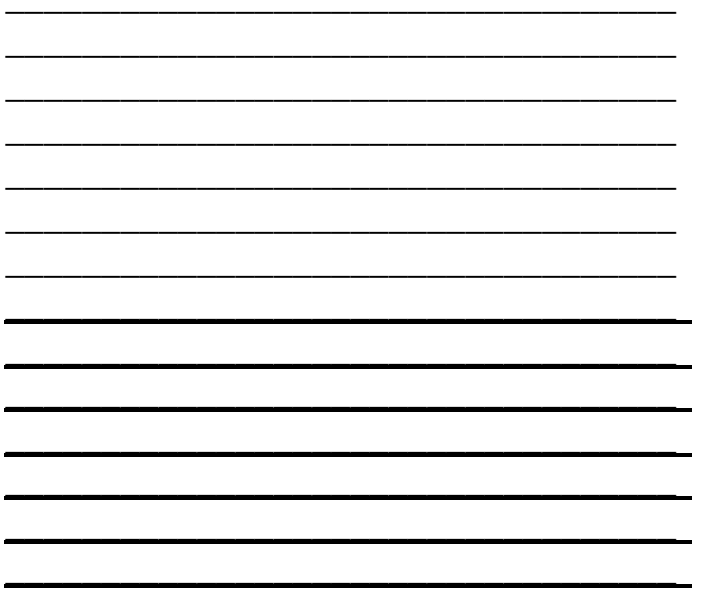

